The Texas Medical Center Library

DigitalCommons@TMC

The University of Texas MD Anderson Cancer Center UTHealth Graduate School of

Biomedical Sciences Dissertations and Theses

(Open Access)
The University of Texas MD Anderson Cancer

Center UTHealth Graduate School of

Biomedical Sciences

\title{
$5-2019$
}

\section{Cross-presentation Is A Source of Tumor Antigens For Multiple Myeloma Immunotherapy}

\author{
Alexander A. Perakis
}

Follow this and additional works at: https://digitalcommons.library.tmc.edu/utgsbs_dissertations

Part of the Biology Commons, Cancer Biology Commons, Immunology and Infectious Disease Commons, and the Medicine and Health Sciences Commons

\section{Recommended Citation}

Perakis, Alexander A., "Cross-presentation Is A Source of Tumor Antigens For Multiple Myeloma Immunotherapy" (2019). The University of Texas MD Anderson Cancer Center UTHealth Graduate School of Biomedical Sciences Dissertations and Theses (Open Access). 949.

https://digitalcommons.library.tmc.edu/utgsbs_dissertations/949

This Dissertation (PhD) is brought to you for free and open access by the The University of Texas MD Anderson Cancer Center UTHealth Graduate School of Biomedical Sciences at DigitalCommons@TMC. It has been accepted for inclusion in The University of Texas MD Anderson Cancer Center UTHealth Graduate School of Biomedical Sciences Dissertations and Theses (Open Access) by an authorized administrator of DigitalCommons@TMC. For more information, please contact digitalcommons@library.tmc.edu.

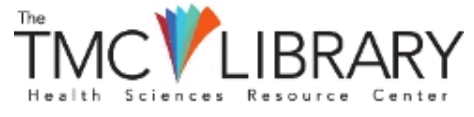




\title{
Cross-presentation Is A Source of Tumor Antigens For Multiple Myeloma Immunotherapy
}

By

\author{
Alexander A. Perakis, B.A.
}

APPROVED:

Gheath Al-Atrash, D.O., Ph.D., Advisory Professor

Jeffrey J. Molldrem, M.D., Advisory Professor

Michelle C. Barton, Ph.D.

Michael A. Curran, Ph.D.

Gregory A. Lizee, Ph.D.

Stephanie S. Watowich, Ph.D.

APPROVED:

Dean, The University of Texas

MD Anderson Cancer Center UTHealth Graduate School of Biomedical Sciences 


\title{
Cross-presentation Is A Source of Tumor Antigens For Multiple Myeloma Immunotherapy
}

\author{
A \\ DISSERTATION \\ Presented to the Faculty of \\ The University of Texas \\ MD Anderson Cancer Center UTHealth \\ Graduate School of Biomedical Sciences \\ In Partial Fulfillment \\ Of the Requirements \\ For the Degree of \\ DOCTOR OF PHILOSOPHY \\ By \\ Alexander A. Perakis, B.A. \\ Houston, Texas \\ May 2019
}




\section{Copyright}

The American Association of Cancer Research (AACR) Journals permits authors to reuse their article or parts of their articles in several ways including "in support of a doctoral thesis or dissertation." The full list of copyrights and permissions granted to authors who publish in AACR journals can be found at the following web address:

http://aacrjournals.org/content/authors/copyright-permissions-and-access 


\section{Dedication}

This work is dedicated to my loving family: My father, Alexander George Perakis, my mother, Patty Perakis, and my sister, Olivia Perakis. Thank you for always loving and supporting me through life. This work is as much mine as it is yours.

I would also like to dedicate this work to my loving friend, Clair Emily Bergeson. I miss you every day. Thank you for bringing so much joy into my life in such a short time and reminding me of the importance of cancer research. 


\section{Acknowledgements}

I would first like to thank my wonderful mentors Dr. Gheath Al-Atrash and Dr. Jeffrey Molldrem. I have been very fortunate to have two mentors that have taught and guided me through graduate school. Thank you Dr. Al-Atrash for everything. You have been an amazing mentor, teacher, and friend. You have taught me so much beyond science. Thank you for always being there for me during these past six years. Thank you Dr. Molldrem for your endless support during graduate school. You always challenged me to know what I know and know what I don't know. I am forever grateful for the wonderful lab you established and let me call home during the past six years.

To my entire lab, you have become my second family. Dr. Celine Kerros, thank you for being nothing short of a big sister to me. Thank you for teaching me accountability, how to trouble shoot the (pardon my French) B.S. in experiments. Thank you for being a shoulder to cry on during the hard times and someone to share laughs with during the good ones. You are a friend for life. Dr. Anne Philips, thank you for all your friendship and mentorship. You have taught me so much and I would not be the scientist I am today without you. Thank you for always willing to talk politics and nerd pop culture with me. Dr. Lisa St. John, thank you for all you do keeping our lab above water. Thank you for being a wonderful friend and lifeline for me. You always made me feel at home. Thank you to Pariya Sukhumalchandra and Mao Zhang for all the hard work and effort you put forth in training and helping me with so many experiments. 
To my advisory committee, Drs. Michael Curran, Gregory Lizee, Stephanie Watowich and Dean Michelle Barton, thank you for all your wisdom and advice throughout the years. You all have shaped me to be the best student and scientist and I am so appreciative of all the time you have invested in my education. To Dean Barton, thank you for all the leadership opportunities you provided be at GSBS. I am continually honored that you believed in me to serve on so many wonderful committees and councils.

To my GSBS friends, thank you all for your friendship and companionship throughout graduate school. It has been a wonderful journey to embark on with all of you. To Erin Atkinson, we started together from the very beginning. You are a friend for life, thank you for filling the years of graduate school with laughs. Chris Evans, thank you for being such a great friend and roommate. To my fellow Graduate Student Association queens, Jill Losh, Kimiya Young and Aundrietta Duncan-thank you for making GSA one of the highlights of graduate school for me. I am so grateful for all of your friendships and the wonderful memories we had together.

Thank you Seth Freeman for reminding me of life outside the lab and for helping me find my way through life. To all my friends back home, Dillon Peters, Tara Peters, Lisa Greene, Justin Gunesch, Tyler Jacobson, thank you for your unconditional love and support throughout life. To Eric Yoon, thank you from the 
bottom of my heart. You have been a wonderful partner. Thank you for all of your unconditional love and support and always lifting me up.

To my incredible family, thank you for always believing in me. Thank you to my loving parents for allowing me to chase my dreams. No matter what obstacles were in front of me, you both were always willing to help get me there. Thank you for all the sacrifices you have made to help Olivia and I be successful. To my best friend in the world and sister, Olivia, thank you for always supporting me. You are an inspiration to me and have always motivated me to be the best version of myself. I am so fortunate to have you by my side to go through life. 


\begin{abstract}
CROSS-PRESENTATION IS A SOURCE OF TUMOR ANTIGENS FOR MULTIPLE MYELOMA IMMUNOTHERAPY
\end{abstract}

\begin{abstract}
Alexander A. Perakis, B.A.
Advisory Professors: Gheath Al-Atrash, D.O., Ph.D., Jeffrey J. Molldrem, MD.
\end{abstract}

Cross-presentation is an essential bridge between the innate and adaptive arms of the immune system where antigen presenting cells (APCs) prime cytotoxic T cell responses. We have recently identified cross-presentation as a mechanism by which solid tumors present exogenous antigens. We therefore hypothesized that multiple myeloma would be capable of cross-presentation as these cells are derived from B cells, known APCs. We explored the capacity of multiple myeloma to cross-present PR1, a human leukocyte antigen (HLA)-A2 nonameric peptide that is derived from neutrophil elastase (NE) and proteinase 3 (P3), and the ability to treat multiple myeloma using PR1-targeting immunotherapies. Here we demonstrate that multiple myeloma cells lack endogenous NE and P3 expression, possess the ability to take up exogenous NE and P3 and cross-present PR1. This process employs the cytosolic antigen presentation machinery including the proteasome, Golgi, and TAP. Subsequent PR1 cross-presentation renders multiple myeloma cells susceptible to PR1-CTL and anti-PR1/HLA-A2 antibody, both in vitro and in vivo. To our knowledge, this is the first report of multiple myeloma cross-presenting tumor antigens. 
Collectively, our data demonstrate that PR1 is a novel tumor antigen in multiple myeloma and can be effectively targeted using PR1-targeting immunotherapies. Our study suggests that the multiple myeloma antigen repertoire is much larger than previously appreciated, and that there is a new catalogue of potential immunotherapeutic targets in multiple myeloma that can be derived from exogenous antigens. 


\section{Table of Contents}

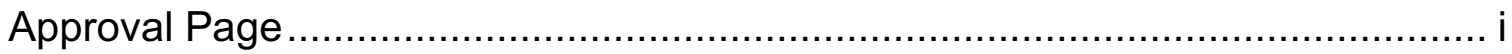

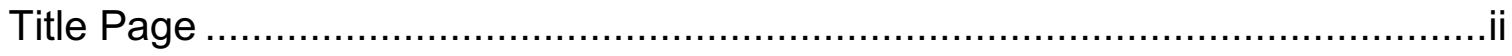

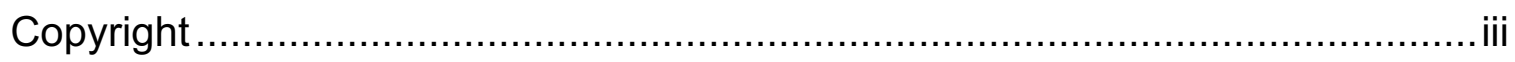

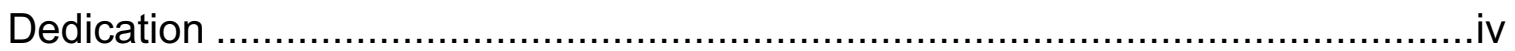

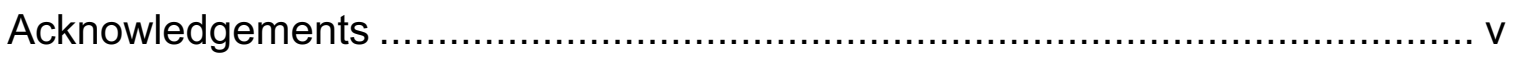

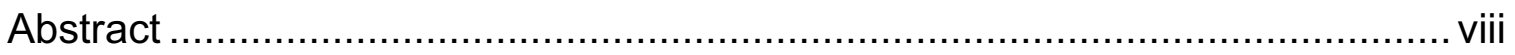

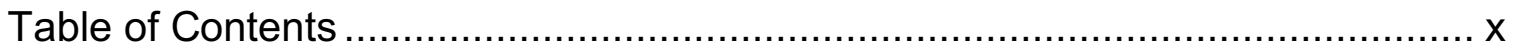

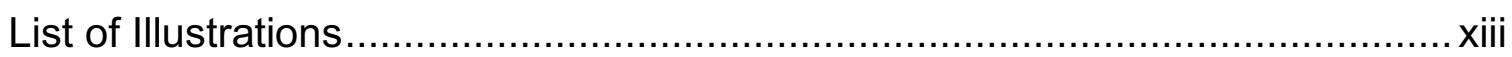

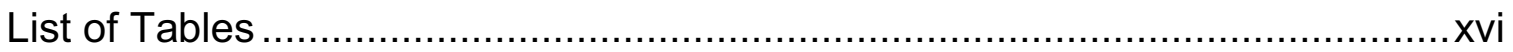

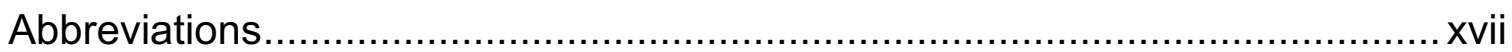

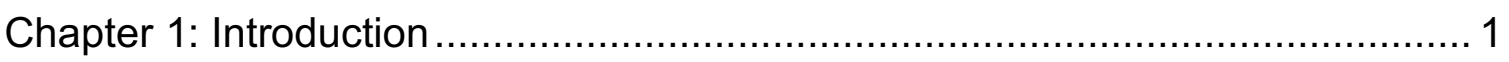

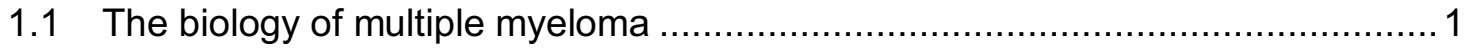

1.2 Immunotherapy for the treatment of multiple myeloma …............................ 6

1.3 Antigen presentation and cross-presentation ............................................. 10

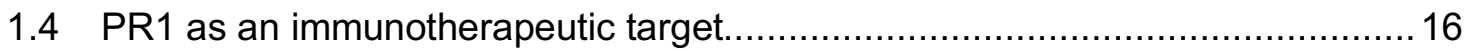

Chapter 2: Materials and Methods............................................................ 19

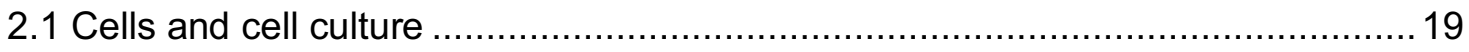

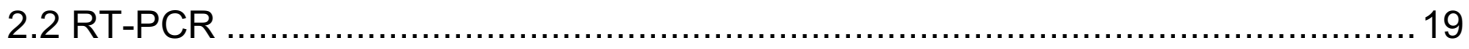




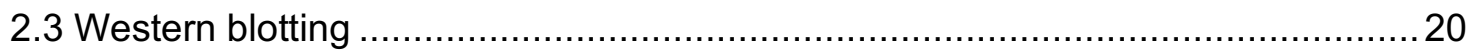

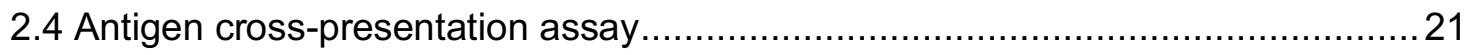

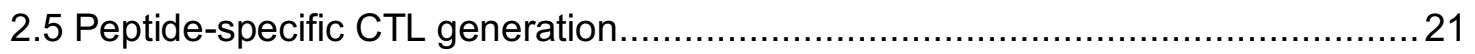

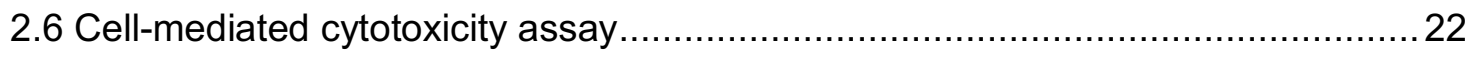

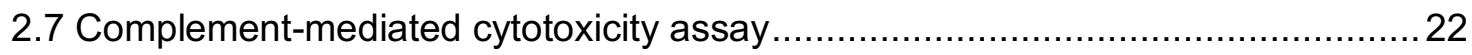

2.8 Staining for PR1-CTL in multiple myeloma patient samples.............................23

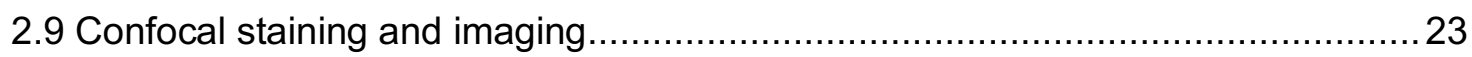

2.10 U266 myeloma xenograft animal model......................................................24

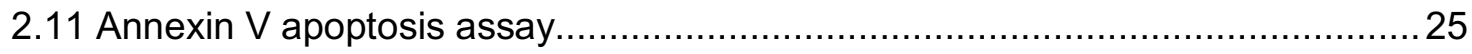

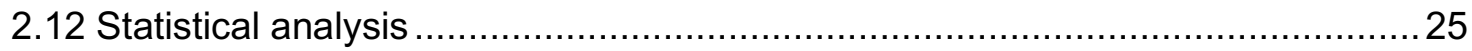

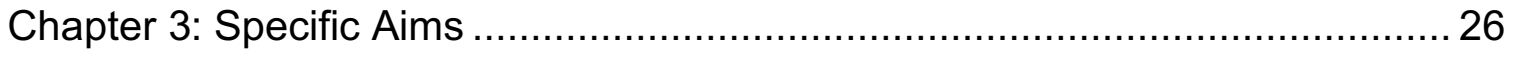

Chapter 4: Determine the extent of NE and P3 uptake and PR1 cross-

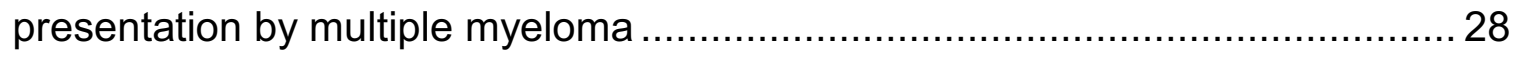

4.1 Multiple myeloma cells lacking endogenous NE and P3 internalize exogenous

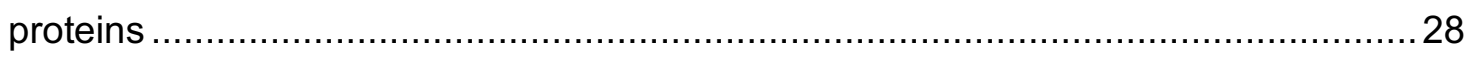

4.2 $\mathrm{HLA}^{-\mathrm{A}^{+}}{ }^{+}$multiple myeloma cells take up and cross-present NE and P3 from

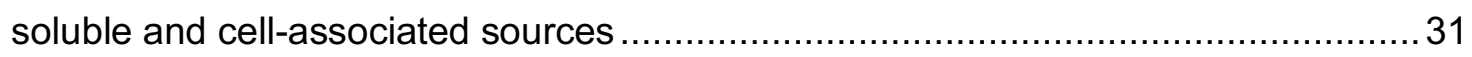

4.3 Cross-presentation by multiple myeloma involves the proteasome and Golgi/ER.

4.4 PR1 cross-presentation by multiple myeloma is TAP-dependent........................54

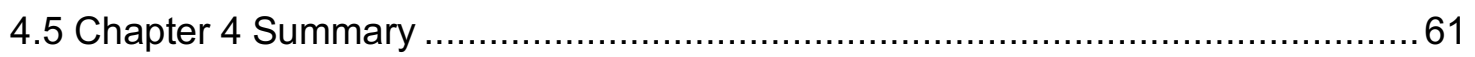

Chapter 5: Evaluate the susceptibility of multiple myeloma to PR1-targeting

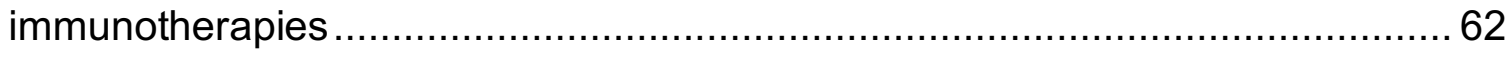


5.1 PR1 cross-presentation increases the susceptibility of multiple myeloma to PR1CTL and 8F4 antibody.

5.2 PR1-CTL and 8F4 antibody reduce multiple myeloma burden in xenograft mice. 66 5.3 8F4 antibody and bortezomib combination therapy results in an increased antimyeloma effect. 76

5.4 PR1/HLA-A2 and PR1-CTL are detected in multiple myeloma patients 81

Chapter 6: Discussion 102

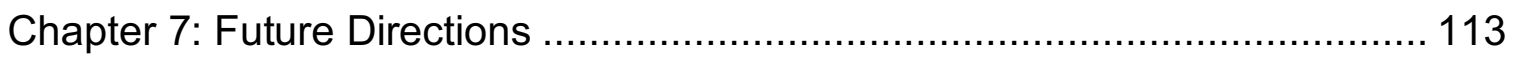

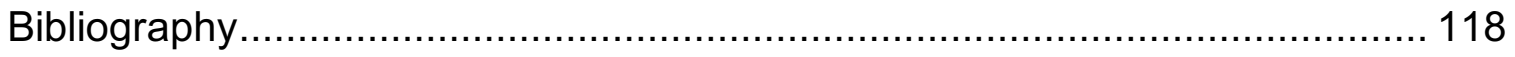

Vita 140 


\section{List of Illustrations}

Figure 1: Overview of the antigen presentation pathway............................... 14

Figure 2. Multiple myeloma cells lack endogenous NE and P3.......................29

Figure 3. Transcript expression of NE and P3 genes in Cancer Cell Line Encyclopedia (CCLE) . ......................................................... 30

Figure 4. Multiple myeloma cells internalize exogenous NE and P3. ............... 32

Figure 5. HLA-A2+ ${ }^{+} 266$ cell line takes up NE and P3 from soluble and cellassociated sources

Figure 6. NE and P3 localize within the cell following co-culture with U266 multiple cells.

Figure 7. HLA-A2 ${ }^{+} \mathrm{U} 266$ cell cross-presents NE and P3 from soluble and cellassociated sources.

Figure 8. Absence of PR1 cross-presentation in HLA-A2 negative multiple myeloma cell lines.

Figure 9. Proteasome and ER/Golgi are involved in the cross-presentation of NE and $\mathrm{P} 3$ by $\mathrm{U} 266$ multiple myeloma cells.

Figure 10. Bortezomib reduces PR1/HLA-A2 expression on the cell surface of U266 multiple myeloma cells.

Figure 11. Lenalidomide does not reduce PR1/HLA-A2 expression on the cell surface of U266 multiple myeloma cells.

Figure 12. HLA-A2 expression on U266 is reduced after addition of proteasome inhibitors 
Figure 13. HLA-A2 expression on U266 is not reduced after addition of brefeldin A or lenalidomide. 52

Figure 14. TAP knock-down in U266 results in decreased PR1/HLA-A2 expression and PR1-mediated killing. 55

Figure 15. APM proteins LMP7, LMP2, and tapasin are upregulated in multiple myeloma patients. 58

Figure 16. Multiple myeloma patients express different isoforms of calnexin. .... 60

Figure 17. PR1 cross-presentation increases the susceptibility of multiple myeloma to PR1-CTL and anti-PR1/HLA-A2 antibody 64

Figure 18. U266 multiple myeloma xenograft model and 8F4 treatment schema.

Figure 19. 8F4 antibody and PR1-CTL reduce multiple myeloma in U266 xenograft mice. 70

Figure 20. Gating strategy for identifying U266 cells in mouse bone marrow..... 72 Figure 21. 8F4 antibody impedes multiple myeloma growth in U266 xenograft mice 73

Figure 22. 8F4 antibody extends survival in U266 xenograft mice 75

Figure 23. 8F4 and bortezomib combination has additive anti-myeloma activity.

Figure 24. 8F4 and bortezomib combination therapy more effectively reduces the multiple myeloma burden in xenograft mice and prolongs survival...79

Figure 25. PR1/HLA-A2 is presented on the surface of patient multiple myeloma cells 83 
Figure 26. U266 cross-presents PR1 after exposure to NE/P3 from PMN coculture.

Figure 27. Gating strategy used to determine PR1-CTL frequency in peripheral blood of multiple myeloma patients following allogeneic stem cell transplantation. 88

Figure 28. PR1 dextramer plots of multiple myeloma patients following allogeneic stem cell transplantation. 90

Figure 29. Phenotype of PR1-CTL in patients following allo-SCT. 92

Figure 30. Gating Strategy used to determine PR1-CTL frequency in peripheral blood of multiple myeloma patients following autologous stem cell transplantation.

Figure 31. Gating Strategy used to determine PR1 expression and co-stimulatory molecule expression on patient bone marrow multiple myeloma cells.

Figure 32: Summary of PR1 cross-presentation by multiple myeloma 112 


\section{List of Tables}

Table 1. Confocal imaging of PR1/HLA-A2 in multiple myeloma patients.

Table 2. PR1-CTL frequency in HLA-A2 ${ }^{+}$multiple myeloma patients following allogeneic stem cell transplantation.

Table 3. PR1-CTL frequency in HLA-A2 ${ }^{+}$multiple myeloma patients following autologous stem cell transplantation.

Table 4. Co-stimulatory molecule expression of PR1/HLA-A2 ${ }^{+}$multiple myeloma patients. 96 


\section{Abbreviations}

\section{F4}

ADCC

ADCP

AML

ANOVA

APM

APC

Allo-SCT

Auto-SCT

$\beta_{2} \mathrm{M}$

BCMA

BM

BiTE

Bor

Car

CAR-T

CD-

CDC

CML

CNX

CRAB
anti-PR1/HLA-A2 TCR-like monoclonal antibody

Antibody Dependent Cellular Cytotoxicity

Antibody Dependent Cellular Phagocytosis

Acute Myeloid Leukemia

Analysis of Variance

Antigen Processing Machinery

Antigen Presenting Cell

Allogeneic Stem Cell Transplant

Autologous Stem Cell Transplant

$\beta_{2}$-microglobulin

B Cell Maturation Antigen

Bone Marrow

Bi-specific T Cell Engager

Bortezomib

Carfilzomib

Chimeric Antigen Receptor T cell

Cluster of Differentiation

Complement Dependent Cytotoxicity

Chronic Myeloid Leukemia

Calnexin

Serum Calcium, Renal insufficiency, Anemia, Lytic Bone Lesions 


\begin{tabular}{|c|c|}
\hline CTL & Cytotoxic T Lymphocytes \\
\hline DC & Dendritic Cell \\
\hline ER & Endoplasmic Reticulum \\
\hline $\mathrm{E}: \mathrm{T}$ & Effector:Target ratio \\
\hline GAPDH & Glyceraldehyde 3-phosphate dehydrogenase \\
\hline G-CSF & Granulocyte Colony Stimulating Factor \\
\hline GvHD & Graft vs. Host Disease \\
\hline GvM & Graft vs. Myeloma \\
\hline HLA & Human Leukocyte Antigen \\
\hline HLA-A2 & Human Leukocyte Antigen-A2*02:01 \\
\hline IL- & Interleukin \\
\hline IMiD & Immune Modulating Drug \\
\hline Lac & Lactacystin \\
\hline Len & Lenalidomide \\
\hline MDSC & Myeloid Derived Suppressor Cell \\
\hline MFI & Median Fluorescence Intensity \\
\hline MGUS & Monoclonal Gammopathy of Undetermined Significance \\
\hline MILs & Myeloma Infiltrating Lymphocytes \\
\hline MM & Multiple Myeloma \\
\hline NE & Neutrophil Elastase \\
\hline NK & Natural Killer Cell \\
\hline NSG & NOD.Cg-Prkd $c^{\text {scid }} \| 2 \operatorname{rg}^{\mathrm{tm} 1 W_{\mathrm{j}} / \mathrm{SzJ}}$ \\
\hline P3 & Proteinase 3 \\
\hline
\end{tabular}




$\begin{array}{ll}\text { PB } & \text { Peripheral Blood } \\ \text { PBMC } & \text { Peripheral Blood Mononuclear Cells } \\ \text { PD-1 } & \text { Programmed Cell Death Protein-1 } \\ \text { PD-L1 } & \text { Program Death-Ligand 1 } \\ \text { PI } & \text { Proteasome Inhibitor } \\ \text { PMN } & \text { Polymorphonuclear Neutrophils } \\ \text { PLC } & \text { Peptide Loading Complex } \\ \text { SEM } & \text { Standard Error of the Mean } \\ \text { SLAMF7 } & \text { Signaling Lymphocytic Activation Molecule F7 } \\ \text { SMM } & \text { Smoldering Multiple Myeloma } \\ \text { Ig } & \text { Immunoglobulin } \\ \text { IgH } & \text { Immunoglobulin Heavy Chain } \\ \text { RT-PCR } & \text { Reverse Transcription-Polymerase Chain Reaction } \\ \text { SCT } & \text { Stem Cell Transplant } \\ \text { siRNA } & \text { Short Interfering RNA } \\ \text { TAP } & \text { Transporter Associated with Antigen Processing } \\ \text { Th- } & \text { Thelper Cell } \\ \text { T-reg } & \text { Wild-Type } \\ \text { UPN } & \text { WT }\end{array}$




\section{Chapter 1: Introduction}

\subsection{The biology of multiple myeloma}

\section{The evolution of multiple myeloma}

Multiple myeloma is characterized by the proliferation of clonal plasma cells in the bone marrow. Normal plasma cells are terminally differentiated B cells, dedicated to the production and secretion of antibodies integral to the humoral response. ${ }^{1}$ Throughout B cell development, these cells undergo frequent error-prone genetic processes, such as VDJ recombination, somatic hypermutation, and Ig heavy chain $(\mathrm{lgH})$ class switching, in order to generate high avidity antibodies. These genetic modification events are prime opportunities for malignant transformation to occur which can lead to the development of multiple myeloma. ${ }^{1,2}$

Multiple myeloma develops from unique multi-step precursor stages where aberrant plasma cells become increasingly malignant. The first stage, monoclonal gammopathy of undetermined significance (MGUS) is asymptomatic and characterized by the presence of serum M-protein, less than $10 \%$ clonal plasma cells residing in the bone marrow and absence of end organ damage that is characterized by serum calcium, renal insufficiency, anemia, lytic bone lesionsCRAB criteria. ${ }^{3}$ While not all cases of MGUS progress to multiple myeloma (approximately $1 \%$ increase per year), it can be appreciated that multiple 
myeloma is frequently preceded by MGUS ${ }^{4,5}$ Smoldering multiple myeloma (SMM) is characterized by more than $10 \%$ of clonal plasma cells residing in the bone marrow with absence of CRAB freatures. ${ }^{3}$ The disease progresses to multiple myeloma when CRAB features are manifested. Occasionally, multiple myeloma can progress to plasma cell leukemia, the most malignant form of the disease. Plasma cell leukemia exists when malignant plasma cells become independent of survival signals from the bone marrow microenvironment and are able to invade the peripheral blood and other organs. ${ }^{1}$ The stepwise process evolution from MGUS to SMM to multiple myeloma is orchestrated through multiple mechanisms involving DNA damaging events, reorganization of the bone marrow (BM) microenvironment, and escaping immune surveillance. This multifocal etiology of multiple myeloma is complex. While this project focuses on the interaction between the immune system and multiple myeloma, the underlying genetic events are important for understanding the origin of this disease.

\section{Oncogenic transformation}

The transformation of normal plasma cells into multiple myeloma involves the acquisition of several different genetic abnormalities. Genetic drivers of multiple myeloma are often classified into primary and secondary events. Primary events involve large-scale genetic changes that include immunoglobulin heavy chain $(\mathrm{lgH})$ translocations, hyperdiploidy, and cyclin D overexpression. ${ }^{1,3}$ Secondary events involve mutations in common oncogenes such as NRAS, 
KRA, BRAF, NF-KB pathway, p53, PTEN, and RB inactivation. ${ }^{3}$ While the genetic abnormalities that occur in multiple myeloma patients are heterogeneous, the initiating events follow a common theme involving gains of chromosomes (such as $5,9,15,19)$ or $\operatorname{lgH}$ translocation events that result in oncogene expression being driven by a strong enhancer region of the immunoglobulin (lg) genes. ${ }^{1}$ The overexpression of the initial oncogenes can promote the accumulation of subsequent mutations leading to the development of multiple myeloma.

\section{Bone marrow microenvironment reorganization}

Genetic sequencing studies have revealed little differences between MGUS and multiple myeloma patients, highlighting the importance of the BM microenvironment in the progression of multiple myeloma. ${ }^{6,7}$ The BM microenvironment is composed of an intricate network of cells (BM stromal cells, osteoblasts, osteoclasts, endothelial cells, lymphocytes, myeloid cells) which play integral roles in hematopoiesis. As mirrored in many other solid tumor types, the BM microenvironment becomes dysregulated and customized to promote myelomagenesis. The bone marrow reorganization is largely due to the effects of a master regulator cytokine, Interleukin-6 (IL-6).

Interactions between myeloma cells and BM stromal cells can lead to the secretion of IL-6 via the NF-KB pathway. IL-6 also influences multiple signaling axes (PI3K/Akt, JAK/Stat, NF-KB, Wnt), all of which become dysregulated to support the growth, survival and drug resistance of multiple myeloma. ${ }^{8,9}$ One of 
the hallmark features of the BM microenvironment transformation mediated by multiple myeloma is the imbalance between osteoclasts and osteoblasts.

Osteoclast activation leads to osteolysis and reduced osteoblast-mediated bone formation..$^{9,10}$

Solo, IL-6 also functions as a potent growth, survival and drug resistance factor and adversely affects the immune cells of the BM microenvironment leading to an immunosuppressive milieu. ${ }^{8}$ The potency of IL-6 in myelomagenesis is highlighted by the observations that when inhibited (via antiIL-6 antibodies) multiple myeloma cell proliferation decreased and cells were resensitized to anti-myeloma drugs, dexamethasone and bortezomib. ${ }^{11}$ However, promising patient outcomes have yet to be observed in clinical trials targeting IL6, suggesting targeting IL-6 alone is not sufficient for the treatment of multiple myeloma. $^{12}$

\section{Immune escape}

Transformation of the immune microenvironment is critical to myeloma pathogenesis. Key components of the immune microenvironment consist of dendritic cells (DC), T cells, and myeloid derived suppressor cells (MDSC). Changes are found in each cell type, which contributes to the establishment of an immunosuppressive environment for multiple myeloma survival. 
DCs have defective antigen presentation abilities in the context of multiple myeloma. This critical process is integral to the stimulation of cytotoxic $T$ cell lymphocytes $(C T L)$. There is a paucity of DCs observed in myeloma patients and furthermore, mature DCs in myeloma patients show reduced expression of costimulatory molecules (CD40 and CD80) and lower expression of HLA-DR. ${ }^{13}$

Due to the plasticity of CD4 T cell development, a process heavily influenced by cytokines, the balance of T cell subsets in multiple myeloma is perturbed. The abundance of IL-4 and IL-10 in the BM microenvironment skews T cell development towards a Th2 phenotype rather than a Th1 phenotype, therefore favoring immune suppression. ${ }^{14}$ The role of T-regulatory cells (T-reg) is less understood in the context of myeloma due to the compartment they are analyzed from (peripheral blood vs. bone marrow) and the methods used for analysis (phenotypic vs functional). Some groups have demonstrated an abundance of T-regs while others have shown low T-reg percentages in the myeloma BM. ${ }^{13,14}$ However, it is well appreciated that the myeloma-derived Tregs are dysfunctional and have profound differences compared to their health counterparts. Due to the effects of IL-6, the balance between Th1, Th17 and Treg cells heavily favors the Th17 phenotype. Th17 cells in turn produce IL-17, which has been shown to promote multiple myeloma cell growth, suppress $\mathrm{T}$ cell anti-tumor activities, and contribute to osteolytic bone disease..$^{13,14}$ 
Additionally, MDSCs contribute to defective T cell mediated immunity. Studies have shown MDSC populations to be elevated in multiple myeloma patients compared to healthy controls. ${ }^{13,15}$ Additionally, these cells produce arginase-1 which directly promotes myeloma tumor growth as well as depletes the extracellular matrix of arginine, an important amino acid for T cell activity. ${ }^{13,14}$ MDSC also aid in myelomagenesis by assisting in the promotion of osteolytic bone disease as these cells can function as osteoclast progenitors. ${ }^{14}$ Key studies have also shown that myeloma cells can induce the development of MDSCs from monocytes, establishing a feedback loop that promotes multiple myeloma progression. ${ }^{15}$

The network between multiple myeloma and the BM microenvironment is critical when considering new treatment strategies of multiple myeloma. This project aims to highlight an important interaction between multiple myeloma and myeloid cells that can be harnessed for new immunotherapy opportunities.

\subsection{Immunotherapy for the treatment of multiple myeloma}

The immune system is heavily implicated in the pathogenesis of multiple myeloma, suggesting many opportunities for therapeutic intervention. This has led to the recently increased interest in applying immunotherapy for the treatment of multiple myeloma. 


\section{Proteasome inhibitors and Immune modulatory drugs}

The introduction of proteasome inhibitors $(\mathrm{PI})$ and immune modulatory drugs (IMIDs) has changed the landscape of myeloma therapy, improving patient survival and outcomes. ${ }^{16,17}$ Proteasome inhibitors (bortezomib, carfilzomib) target the proteasome and inhibit intracellular protein degradation, causing cell cycle arrest through the endoplasmic reticulum (ER) stress response and apoptosis through the activation the unfolded protein response and activation of caspse- 8 and $9 .{ }^{18,19}$ Bortezomib is a boronate PI that inhibits the $\beta 5$ subunit of the proteasome and was the first PI to see clinical success for the treatment of myeloma. Now, next generation PIs, carfilzomib and ixazomib, are currently being investigated in clinical trials and show promise as more potent options to bortezomib. ${ }^{20}$ Since bortezomib affects the proteasome, a key component in antigen presentation, we further investigated how bortezomib affects the ability of multiple myeloma to cross-present antigens.

IMiDs (thalidomide, lenalidomide, pomalidomide) have multiple mechanisms of action that include both augmenting the immune system and direct anti-myeloma activity. These mechanisms involve promoting the production of IL-2, interferon (INF) y and other pro-inflammatory cytokines that activate T cells and natural killer (NK) cells. ${ }^{21}$ Lenalidomide has also been demonstrated to induce the expression of DC maturation markers to promote antigen presentation to T cells. ${ }^{22}$ Currently, lenalidomide, in combination with other myeloma therapies is one of the standard of care treatments for newly 
diagnosed patients. ${ }^{23}$ In this present work, we sought to determine how lenalidomide could modulate cross-presentation by multiple myeloma.

\section{Allogeneic stem cell transplant}

While multiple myeloma remains incurable with standard therapies, which include autologous stem cell transplant (auto-SCT), allogeneic stem cell transplant (allo-SCT) has remained the only curative option for patients. This treatment strategy relies on a potent conditioning chemotherapy regimen that ablates the patient's bone marrow, followed by the infusion of stem cells (and other cellular components) from an HLA-matched-healthy donor to achieve a graft vs. myeloma (GvM) effect. The difficulty and limitation of this therapy comes from its highly toxic side effects such as graft vs. host disease (GvHD), where donor-derived T cells mount an immune response that targets host normal tissues. Oftentimes, the benefits of GvM are outweighed by the toxicity of alloSCT, which includes the side effects of the conditioning regimen as well as GvHD. Additionally, multiple myeloma is more often diagnosed in elderly patients who are more susceptible to the side effects of the myeloablative conditioning regimens, limiting this therapy to younger and healthier patients. ${ }^{24,25}$ Therefore, there is an interest in identifying new immunotherapies, that offer a more targeted approach and less side effects, that can be more widely used to treat multiple myeloma patients. 


\section{Targeting multiple myeloma antigens with immunotherapies}

Currently the most promising immunotherapies used to treat multiple myeloma target myeloma-associated antigens. There are only a handful of myeloma-specific antigens, most commonly cancer testis antigens (MAGE-A3 and NY-ESO-1). These targets are under current investigation in ongoing clinical trials. ${ }^{26}$ Most of the current immunotherapies target myeloma-associated antigens, proteins and antigens that are enriched on the surface of myeloma and plasma cells. The immunotherapy modalities currently under investigation are similar to the immunotherapies being used in other tumor settings such as monoclonal antibodies, chimeric antigen receptor T cells (CAR-T), bispecific $T$ cell engagers (BiTE), and immune checkpoint inhibition.

To date, two monoclonal antibodies are clinically FDA approved for the treatment of multiple myeloma: daratumumab (targets CD38) and elotuzumab (targets SLAMF7). CD38 is highly expressed on plasma cells, NK cells, and myeloid cells. Daratumumab has multiple methods of activity including complement-dependent cytotoxicity (CDC), antibody-dependent cytotoxicity (ADCC) as well as Treg and MDSC-inhibition..$^{27,28}$ Similarly elotuzumab also has ADCC activity and can activate NK cells as SLAMF7 is highly expressed on both plasma cells and NK cells. ${ }^{29}$ CAR-T cells are genetically engineered T cells that have the antigen recognition regions of a monoclonal antibody linked to the TCR domains and co-stimulatory domains, resulting in a T cell that can directly recognize an antigen of choice. CAR-T cells have been used in the setting of 
multiple myeloma, targeting CD19 and B cell maturation antigen (BCMA).

Additionally, CD19 has been targeted using BiTE and preclinical studies have investigated targeting CD38 and CD138. ${ }^{30}$ Immune checkpoint inhibition through the use of blocking antibodies is also under investigation in multiple myeloma. Program death-ligand 1 (PD-L1) expression is upregulated on myeloma cells in patients and its receptor, programmed cell death protein-1 (PD-1) expression is elevated on the T cells and NK cells. ${ }^{31}$

While there are a number of multiple myeloma targets and different immunotherapeutic strategies under investigation, many of these aforementioned treatments have yielded promising but limited clinical results, mainly as they fail to fully eradicate the underling multiple myeloma. Thus, there is a continued need for further investigation into improving the current modalities and searching for novel immunotherapies.

\subsection{Antigen presentation and cross-presentation}

While the exact mechanisms of how multiple myeloma avoids immune destruction remain unclear, it is clear that $\mathrm{CD} 8^{+} \mathrm{T}$ cells fail to manage the outgrowth and expansion of multiple myeloma cells. A critical step in T cell activation occurs through the ability of both dendritic cells and tumor cells to present antigen. Moreover, downregulation of antigen presentation is a common strategy employed by tumor cells to avoid immune detection. ${ }^{32}$ Therefore, in this 
present work we focused on investigating the biology of antigen presentation in multiple myeloma.

\section{Human leukocyte antigen system}

Antigen presentation is the process by which antigens, usually proteins, are degraded intracellularly into small peptide fragments that are displayed on the cell surface in the context of human leukocyte antigen (HLA) class I and class II molecules for recognition by T cells. HLA class I is a heterodimer cell surface molecule that consists of a $\alpha$-heavy chain which is encoded by the highly polymorphic HLA gene (HLA-A, -B, and -C) and $\beta_{2}$-microglobulin, and presents antigens recognized by $C D 8^{+} T$ cells. HLA class II molecules (HLA-DM, -DP, $D O,-D Q,-D R)$ are also heterodimers, but consist of a $\alpha$-chain and $\beta$-chain-both of which are encoded by HLA genes and presents antigens to $\mathrm{CD} 4^{+} \mathrm{T}$ cells. The polymorphisms of the HLA genes allows for fine-tuning of the adaptive immune responses and plays a major role in hematopoietic and solid organ transplantation. The HLA-A2 haplotype has garnered interest due to having the highest frequency among the Caucasian population. Importantly, HLA class I downregulation does not appear to be a common immune escape mechanism in multiple myeloma, as it can be for other tumor types. However, Racanelli and colleagues have demonstrated that while antigen presentation in multiple myeloma cells is altered, many of the components of the antigen presentation machinery (APM) were upregulated in multiple myeloma patients. ${ }^{33}$ Additionally, 
work from our group has suggested that HLA class I is abundantly expressed on the cell surface of multiple myeloma. ${ }^{33,34}$

\section{Antigen presentation pathway}

The mechanism by which HLA molecules present antigens to the immune system is accomplished through an organized, step-wise process known as the antigen presentation pathway (Figure 1). Classically, HLA class I molecules display antigens that are expressed endogenously in the cytosol. Intracellular proteins are first targeted for degradation by the proteasome, which facilitates the generation of small, 8-10 amino acid peptides that are favored for HLA class I binding. Unfolded, immature HLA class I heavy chain molecules reside in the ER and are stabilized by the chaperon protein-calnexin (CNX) before binding to $\beta_{2-}$ microglobulin $\left(\beta_{2} M\right)$. Upon $\beta_{2} M$ binding, HLA molecules are stabilized and subsequently released from CNX. Next, HLA binds to the peptide-loading complex (PLC) consisting of calreticulin, tapasin and transporter associated with antigen processing protein 1 and 2 (TAP1/2).

The peptides are transported from the cytosol into the ER via the TAP1/2 protein where they interact with the PLC and HLA. The PLC orchestrates both peptide editing and binding to HLA to allow for proper binding to HLA. Once a high affinity peptide binds to the peptide groove of HLA, the HLA molecule is released from the PLC and shuttled through the ER to Golgi transport and displayed on the cell surface to interact with $\mathrm{CD} 8^{+} \mathrm{T}$ cells. While this pathway 
facilitates endogenous peptide recognition, exogenous peptides can also be displayed to cytotoxic T cells via the cross-presentation pathway. 
Figure 1: Overview of the antigen presentation pathway.

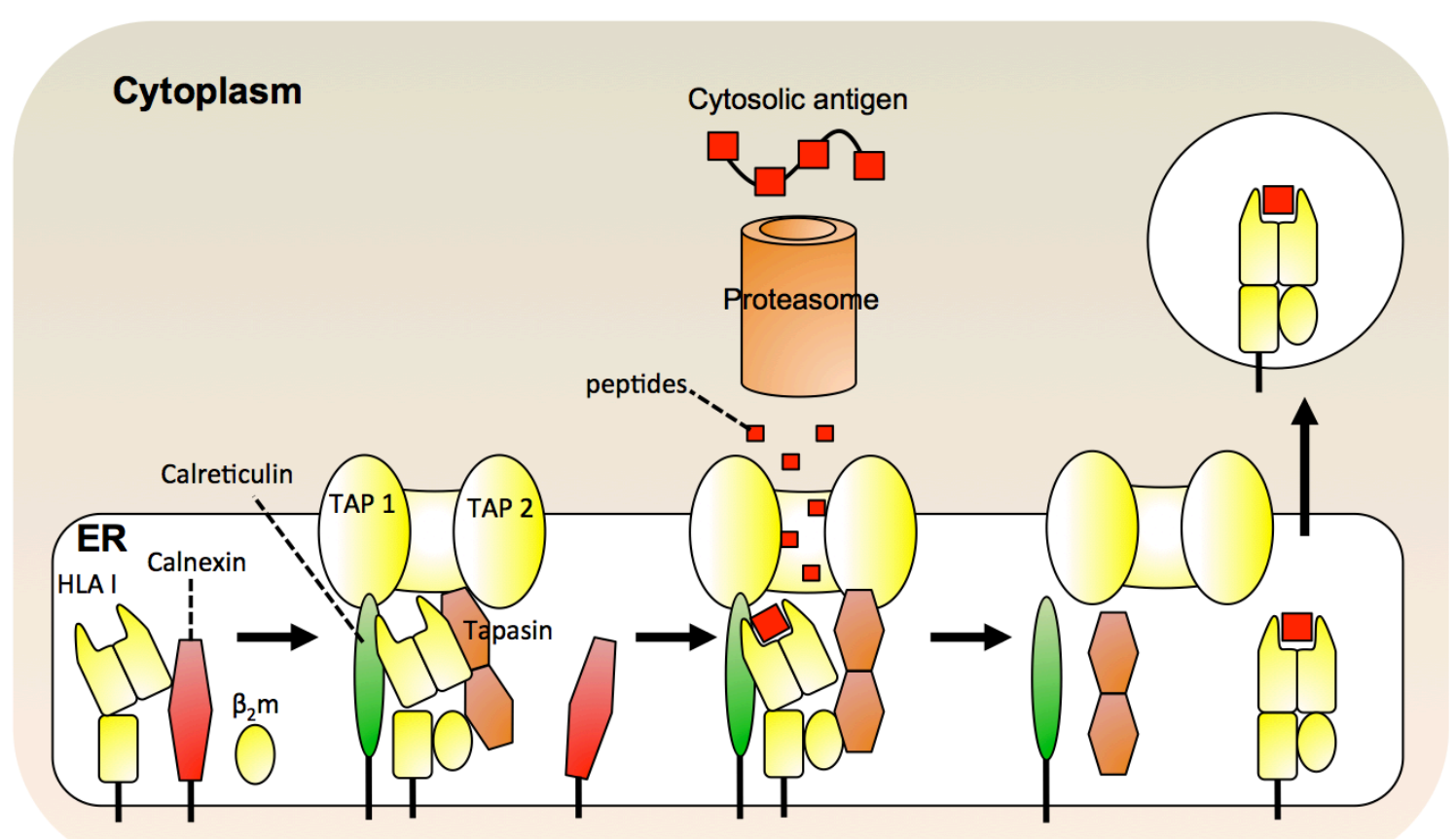

Figure 1: Overview of the antigen presentation pathway. Unfolded HLA class I molecules reside in the ER, stabilized by calnexin. Upon $\beta_{2} \mathrm{M}$ binding, HLA class I molecules bind to the peptide-loading complex composed of calreticulin, TAP1/2, and tapasin. Endogenous antigens are loaded onto HLA class I molecules after being processed by the proteasome in the cytoplasm. Antigens are then transported into the ER lumen via TAP1/2 where they bind to HLA class I. Upon binding a high affinity peptide, HLA class I molecules are then shuttled to the cell surface through the endocytic pathway. 


\section{Cross-presentation}

Cross-presentation is integral to the anti-tumor immune response as it allows DC priming of naïve $\mathrm{CD} 8^{+} \mathrm{T}$ cells against exogenous antigens. Much of how exogenous antigens are processed and cross-presented mirrors the pathway of how endogenous antigens are presented with a few notable unique characteristics. ${ }^{35}$

The defined means by which peptides are initially transported from the microenvironment into the cytosol remains an area of active research. There are three popular models that describe cross-presentation. The first model is the cytosolic pathway model. In this model, the antigen is internalized in a phagosome through phagocytosis. In the phagosome, the antigen can be exported to the cytosol for proteasomal degradation. The processed antigen can then either be loaded onto HLA class I molecules in the ER (similar to classical antigen presentation) or re-imported into the phagosome which can contain its own PLC and HLA class I molecules. ${ }^{36-38}$ Alternatively, in the vacuolar model, internalized antigens are processed by phagosomal degradation and loaded onto HLA class I molecules in the phagosome. ${ }^{36}$ The peptide exchange model describes another possible mechanism of how exogenous peptides are imported into the cell. In this model, surface HLA class I molecules that have already bound peptide are recycled back into the phagosome where old peptides can exchange for newly generated exogenous peptides. ${ }^{37,38}$ HLA class I reinternalization is facilitated through monoubiquitination of a conserved lysine 
residue (tyrosine in mouse) in the cytosolic tail of the HLA class I molecule. ${ }^{37,39}$ Importantly, the peptide exchange model offers a TAP-independent mechanism for exogenous peptide loading.

Cross-presentation has previously been limited to hematopoietic cells, specifically professional antigen presenting cells (APC; notably DC, macrophages, and B cells). However, recent work from our group has demonstrated that solid tumor cells (breast cancer, melanoma, lung cancer) are capable of cross-presentation. ${ }^{40-44}$ In the case of multiple myeloma, we hypothesized multiple myeloma can cross-present antigen, due to their hematopoietic lineage.

\subsection{PR1 as an immunotherapeutic target}

\section{Neutrophil elastase, proteinase 3, and PR1}

Neutrophil elastase (NE) and proteinase 3 (P3) are two myeloid azurophil granule specific serine proteases expressed by myeloid progenitor cells, polymorphonuclear leukocytes (PMNs) and monocytes. NE and P3 possess normal antimicrobial effects in the context of inflammation. These serine proteases reside in the azurophil granules; specialized lysosomes within the neutrophil, where pathogenic proteins are hydrolyzed. Additionally, NE can be secreted from the neutrophil into the extracellular matrix during inflammatory conditions. ${ }^{45}$ 
PR1 (sequence: VLQELNVTV) is a nonameric HLA-A*0201 (HLA-A2) restricted peptide derived from NE (amino acids 168-176) and P3 (amino acids 128-136).$^{46}$ PR1 was originally discovered and characterized from acute myeloid leukemia (AML) and chronic myelogenous leukemia (CML). In these tumor settings, NE and P3 are aberrantly expressed, making PR1 an attractive tumor antigen for immunotherapy. ${ }^{41,46-48}$ While this would appear to limit PR1 as a therapeutic target to only hematologic malignancies that aberrantly express NE and P3, there is a preponderance of work from our group that suggests PR1 can be targeted in other tumor types via cross-presentation of NE and P3. Prior to this current work, our group has demonstrated that non-myeloid tumor types (breast cancer, melanoma, lung cancer) can take up and internalize NE/P3 from neutrophils and cross-present PR1 in the context of HLA-A2. ${ }^{40-44}$

\section{PR1-targeting immunotherapies}

We first demonstrated the feasibility of targeting PR1 with immunotherapy in patients with relapsed/refractory AML, CML, and myelodysplastic syndrome using a PR1-peptide vaccine and demonstrated that vaccination can achieve objective clinical responses. ${ }^{49}$ We further developed a T-cell receptor (TCR)-like monoclonal antibody that recognizes the PR1/HLA-A2 complex (8F4 antibody). The 8F4 antibody is unique in that it recognizes the combined epitope of the PR1/HLA-A2 complex, mimicking the manner by which TCRs recognize peptide:HLA complexes. However, $8 \mathrm{~F} 4$ retains the high binding affinity of an antibody (dissociation constant $\left.\left[K_{D}\right]=9.9 n M\right) .50,51$ 
8F4 has shown broad anti-leukemia activity against AML cell lines, primary AML patient samples, and patient derived xenograft models. Specifically, our group has illustrated that $8 \mathrm{~F} 4$ antibody can induce complement-dependent cytotoxicity (CDC) and antibody-dependent cellular cytotoxicity (ADCC) against AML in vitro and specifically inhibits growth of AML progenitor cells..$^{50}$ Furthermore, we have also shown that $8 \mathrm{~F} 4$ has anti-leukemia functionality against primary human AML cells in vivo by reducing the leukemia burden, extending survival, and inhibiting AML engraftment in human xenograft mouse models. ${ }^{52}$ Based on the success of this work $8 \mathrm{~F} 4$ is currently being tested in an ongoing Phase I/II clinical trial.

Based on the unique characteristics of multiple myeloma and its microenvironment, we hypothesize that multiple myeloma can take up NE and P3 and subsequently cross-present the PR1 peptide on the cell surface, rendering multiple myeloma susceptible to PR1-targeting immunotherapies. This hypothesis is based on the observation that multiple myeloma is a malignant plasma cell, which originates from the B cell lineage (a known APC), suggesting these cells will be capable of cross-presentation. Additionally, the bone marrow microenvironment, where multiple myeloma homes to, is rich in myeloid progenitor cells and PMNs, providing an abundant source of NE and P3 for PR1 cross-presentation. 


\section{Chapter 2: Materials and Methods}

The following chapter is based on my publication, used with permission granted by AACR Journals.

Alatrash, G. ${ }^{1}$, Perakis, A.A. ${ }^{1}$, Kerros, C., Peters, H.L., Sukhumalchandra, P., Zhang, M., Jakher, H., Zope, M., Patenia, R., Sergeeva, A., Yi, S., Young, K.H., Philips, A.V., Cernosek, A.M., Garber, H.R., Qiao, N., Weng, J., St John, L.S., Lu, S., Clise-Dwyer, K., Mittendorf, E.A., Ma, Q. \& Molldrem, J.J. Targeting the Leukemia Antigen PR1 with Immunotherapy for the Treatment of Multiple Myeloma. Clin Cancer Res 24, 3386-3396 (2018).

\subsection{Cells and cell culture}

Arkansas (ARK), LP-1, ARP-1, IM-9, OPM-2, RPMI 8226 and U266 multiple myeloma cell lines, U937 histiocytic leukemia cell line, T2 T/B cell hybridoma cell line, H2023 lung cancer cell line, and T-47D and MDA-MB-453 breast cancer cell lines were all acquired from ATCC. All cell lines were cultured in RPMI 1640 media with $25 \mathrm{mmol} / \mathrm{L}$ HEPES + I-glutamine (Hyclone) and supplemented with $10 \%$ fetal bovine serum (Gemini Bio-Products), $100 \mathrm{U} / \mathrm{mL}$ penicillin, and $100 \mu \mathrm{g} / \mathrm{mL}$ streptomycin (Cellgro). All cell lines were cultured in $5 \%$ $\mathrm{CO}_{2}$ incubator at $37^{\circ} \mathrm{C}$. All cell lines were thoroughly verified using short tandem repeat DNA fingerprinting performed at the MD Anderson Sequencing and Microarray Facility.

\subsection{RT-PCR}

Cell mRNA was purified from cell lines using RNA Stat 60 kit (TelTest) and cDNA was synthesized using Gene AMP RNA kit (Perkin Elmer). Amplification of 
cDNA was performed using iCycler (Bio-Rad) using the following primer sets: NE forward primer 5'-CACGGAGGGGCAGAGACC-3' and NE reverse primer 5'-

TATTGTGCCAGATGCTGGAG-3'; P3 forward primer 5'-

GACCCCACCATGGCTCAC-3' and P3 reverse primer 5'-

ATGGGAAGGACAGACAGGAG-3'; actin forward primer 5'-

CCAGAGCAAGAGAGCTATCC-3' and actin reverse primer 5'-

CTGTGGTGGTGAAGCTGTAG-3'. ${ }^{40,53}$ DNA was separated on $1.5 \%$ agarose gel and detected using GelDoc2000 (Bio-Rad).

\subsection{Western blotting}

Cell lysates were prepared for western blotting by resuspending cell pellets in lysis buffer (10 mM/L HEPES [pH 7.9], $10 \mathrm{mM} / \mathrm{L} \mathrm{KCl,} 0.1 \mathrm{mM} / \mathrm{L}$ EGTA 0.1mM/L EDTA, $1 \mathrm{mM} / \mathrm{LDTT}$ ) containing proteases inhibitors (Thermo Fisher Scientific). Lysis reaction was performed for 30 minutes at $4^{\circ} \mathrm{C}$. Protein concentrations were calculated using standard Bradford assay (Bio-Rad). Protein separated on $10 \%$ SDS gels (Bio-Rad) using electrophoresis under reducing conditions and transferred onto polyvinylidene fluoride membranes. Prior to immunoblotting, membranes were blocked with $5 \%$ milk solutions in 0.05\% TBS-Tween. Membranes were probed using antibodies against NE (Santa Cruz Biotechnology), P3 (NeoMarkers), actin (Millipore), GAPDH (Millipore), Calnexin, LMP2, LMP7, Tapasin (all Cell Signaling), TAP1 (Enzo Life Sciences), Pan-HLA (HC-10 hybridoma) and peroxidase-conjugated secondary antibodies 
(Jackson ImmunoResearch). Chemiluminescence signal was measured on film (Kodak) and digitally using ChemiDoc Touch Imaging System (Bio-Rad).

\subsection{Antigen cross-presentation assay}

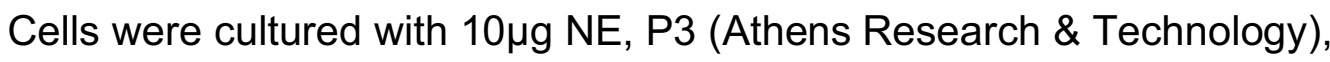
EndoGrade ovalbumin (Ova; Hyglos) or irradiated PMNs or peripheral blood mononuclear cells (PBMCs; 1:1 ratio) at varying durations. Cells were permeabilized by washing in Perm/Wash buffer (BD Biosciences) and stained with fluorochrome-conjugated antibodies (Invitrogen) against P3 (Thermo Scientific) or NE (Santa Cruz Biotechnology). Cross-presentation of peptides was measured by staining with fluorescently conjugated anti-PR1/HLA-A2 antibody (8F4). Inhibition of cross-presentation was accomplished by treating cell cultures with the endoplasmic reticulum (ER) to Golgi anterograde inhibitor, brefeldin A (Sigma-Aldrich), or proteasome inhibitors, lactacystin (Sigma-Aldrich) or bortezomib (Millennium Pharmaceuticals). To determine the effect of IMiDs on cross-presentation, cell cultures were treated with lenalidomide (Celgene).

\subsection{Peptide-specific CTL generation}

PR1-specific CTL were generated from HLA-A2 healthy donor PBMC by stimulating with PR1 peptide (Bio-synthesis Inc.) in vitro. PBMC from healthy donor leukapheresis were isolated using Histopaque 1077 gradient centrifugation (Sigma-Aldrich) and were co-cultured with PR1 $(20 \mu \mathrm{g} / \mathrm{mL})$-pulsed T2 cells at 1:1 
ratio in RPMI 1640 media supplemented with 10\% human AB serum (Gemini Bio-Products). Cell cultures were restimulated with PR1-pulsed T2 cells and 20 $\mathrm{IU} / \mathrm{mL}$ of recombinant human IL-2 (Biosource International) on days 7,14 , and 21.

\subsection{Cell-mediated cytotoxicity assay}

Target cells $\left(1 \times 10^{3}\right.$ cells $\left./ \mathrm{mL}\right)$ were fluorescently labeled with calcein-AM (Invitrogen) for 15 minutes at $37^{\circ} \mathrm{C}$ and then thoroughly washed with RPMI 1640 to remove free calcein-AM. Target cells were co-cultured with peptide specific $\mathrm{CTL}$ at indicated effector-to-target $(\mathrm{E}: \mathrm{T})$ ratios for 4 hours at $37^{\circ} \mathrm{C}$ in 60 well Terasaki plates. Trypan blue was added to each well to stop the reaction. The fluorescence was measured on a CytoFluor II plate reader (Applied Biosystems). The percent cytotoxicity was calculated as follows:

$$
\begin{aligned}
& \left(\left(1-\text { fluoresence }_{\text {target }+ \text { effector }}-\text { fluoresence }_{\text {media }}\right) / \text { fluoresence }_{\text {target alone }}-\right. \\
& \text { fluoresence } \left.\left._{\text {media }}\right)\right) \times 100
\end{aligned}
$$

\subsection{Complement-mediated cytotoxicity assay}

U266 cells ( $1 \times 10^{6}$ cells) were cultured with soluble NE or P3 for 24 hours and then labeled with calcein AM. U266 cells were resuspended in serum-free RPMI 1640 media and treated with anti-PR1/HLA-A2 antibody (8F4) or isotype control antibody for 10 minutes at $37^{\circ} \mathrm{C}$. Standard rabbit complement ( $\left.\mathrm{C}^{\prime}\right)$ (Cedarlane Labs) was added and cells were incubated for 60 minutes at $37^{\circ} \mathrm{C}$. Fluorescence was measured and cytotoxicity was measured as described in section 2.6. 


\subsection{Staining for PR1-CTL in multiple myeloma patient samples}

Patient and HD peripheral blood (PB) samples and $\mathrm{BM}$ aspirates were collected after informed consent to participate in an MD Anderson Cancer Center institutional review board-approved study. PBMC were isolated using Histopaque 1077 gradient centrifugation (Sigma-Aldrich). PBMC were stained using the following fluorescent antibodies: CD8 APC-H7 (BD), CD3 FITC (BD), PEconjugated PR1/HLA-A2-dextramer (Immudex) or tetramer (Baylor College of Medicine MHC Tetramer Core (Houston, TX) and the following pacific blue conjugated lineage antibodies: CD4 (BD), CD14 (BD), CD16 (BD) and CD19 (Biolegend). Samples were fixed with $4 \%$ paraformaldehyde. Data were acquired

on a Canto flow cytometer (BD Biosciences) and analyzed using FlowJo software (Tree Star). The frequency of PR1-CTL was determined as the percentage of live cells that were lineage $, \mathrm{CD}^{+}, \mathrm{CD} 8^{+}$and PR1-dextramer ${ }^{+}$or tetramer ${ }^{+}$.

Phenotype of PR1-CTL (PR1/HLA-A2-dextramer ${ }^{+}$) was investigated using CCR7 PE-Cy7 (Biolegend) and CD45RA PerCP-Cy5.5 (Biolegend) staining, and was classified as central memory (CCR7 $\left.{ }^{+} / \mathrm{CD} 45 \mathrm{RA} \mathrm{A}^{-}\right)$, effector memory (CCR7/CD45RA'), naïve $\left(\mathrm{CCR}^{+} / \mathrm{CD}^{-} 5 \mathrm{RA}^{+}\right)$or terminally differentiated (CCR7/CD45RA ${ }^{+}$.

\subsection{Confocal staining and imaging}

Bone marrow smears and U266 cells were fixed with cold acetone and blocked with 5\% normal mouse serum (Jackson ImmunoResearch). Fixed slides were washed with PBS and then double stained with Alexa-647 conjugated 8F4 
antibody and Alexa-488 conjugated mouse anti-human HLA-A2 antibody (Serotec) or Alexa-488 conjugated rabbit anti-CD138 antibody (Bioss). Slides were stained with antibodies for 90 minutes at room temperature. After washing, ProLong Gold antifade reagent with DAPI (Invitrogen) was added. Confocal imaging was performed using Leica Microsystems SP2 SE confocal microscope with $10 x / 25$ air, $63 x / 1.4$ oil objectives. Leica LCS software (version 2.61) was used for image analysis.

\subsection{U266 myeloma xenograft animal model}

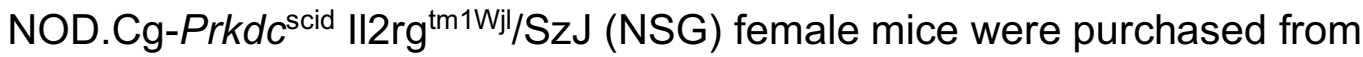
Jackson Laboratory and housed at MDACC following International Animal Care and Use Committee-approved protocol. Mice received sublethal irradiation 24 hours prior to intravenous injection with $2 \times 10^{6}$ U266 cells ${ }^{54}$. U266 engraftment and disease burden was measured by quantifying blood human IgE level by ELISA (Bethyl Laboratories). Mice were treated intravenously with $0.5 \times 10^{6}$ PR1CTL, irrelevant peptide (CG1, E75)-CTL or left untreated (PBS-treated) 14 days after U266 engraftment. Mice were treated intravenously with 8F4 antibody $(10 \mathrm{mg} / \mathrm{kg})$ or $\operatorname{lgG} 1$ isotype control (Herceptin, $10 \mathrm{mg} / \mathrm{kg}$ ) three-times per week beginning on day 28 for a total of 10 injections. ${ }^{52}$ Mice that received bortezomib (Millennium Pharmaceuticals) or carfilzomib (Sellek Chemicals) received four weekly intravenous injections of the respective proteasome inhibitor $(0.025$ $\mathrm{mg} / \mathrm{kg}$ ). Mice were sacrificed 35 days after CTL infusion or 3-4 days after last antibody treatment, and bone marrow was harvested, stained with mouse CD45, 
human (h) CD45, hCD138, and HLA-A2 fluorescently conjugated antibodies and then analyzed by flow cytometry.

\subsection{Annexin V apoptosis assay}

U266 cells were co-cultured with irradiated PMN (1:3 ratio) for 24 hours prior to experimentation. The next day, U266 $\left(2 \times 10^{5}\right.$ per well) were seeded into a 96-well plate and resuspended in fresh medium. U937-WT (U937-wild type) and U937-A2 (U937-HLA-A2 transduced) cells were used as negative and positive controls respectively. Cells were treated with either $8 \mathrm{~F} 4$ alone $(2 \mathrm{mg} / \mathrm{mL})$, bortezomib alone (2.5 nM, Millennium Pharmaceuticals) or in combination overnight at $37^{\circ} \mathrm{C}$. Cross-linking antibody Fab fragments were added to cells (Jackson ImmunoResearch) to facilitate 8F4 mediated apoptosis. Cells were then washed in PBS and intracellularly stained for Annexin V (BD Biosciences), propidium iodide (BD Biosciences), and anti-CD138 (Biolegend) to identify U266 cells in a 96 well plate. Cells were analyzed on a LSR Fortessa (BD Biosciences) and FlowJo software (Tree Star). Apoptosis percentages were calculated by adding the Annexin $\mathrm{V}^{+}$and Annexin $\mathrm{V}^{+} \mathrm{PI}^{+}$gates.

\subsection{Statistical analysis}

All statistical analyses were calculated using GraphPad Prism 6.0 and 7.0 software. Significance was determined for $p$ values less than 0.05 . 


\section{Chapter 3: Specific Aims}

\section{Aim 1: Determine the extent of NE and P3 uptake and PR1 cross- presentation by multiple myeloma}

Cross-presentation of extracellular proteins has been demonstrated in several other tumor types (breast cancer, melanoma, lung cancer). ${ }^{40-44}$ Since multiple myeloma differentiates from the B cell, a known APC, we hypothesize that multiple myeloma has the capability to take up NE and P3 and subsequently cross-present the PR1 peptide on the cell surface. Lack of endogenous transcript and protein expression of NE and P3 will be determined in multiple myeloma cell lines by RT-PCR and western blot analysis respectively. The ability of multiple myeloma cell lines to take up NE and P3 from soluble and cellular sources will be determined using flow-based assays by staining for internalized NE and P3 protein. Cross-presentation of PR1 by multiple myeloma will be measured by staining for PR1/HLA-A2 surface expression using the 8F4 antibody.

\section{Aim 2: Evaluate the susceptibility of multiple myeloma to PR1-targeting immunotherapies}

PR1-targeting immunotherapies have been utilized with great success for the treatment of myeloid malignancies. ${ }^{40,49,50,52,55-58}$ We hypothesize that crosspresentation of PR1 will render multiple myeloma susceptible to immunotherapies targeting PR1. Susceptibility to PR1-targeting immunotherapies 
will be investigated using PR1-specific CTL and 8F4 antibody. The HLA-A2 ${ }^{+}$ U266 multiple myeloma cell line will be co-cultured with NE and P3 to facilitate cross-presentation of PR1 and will be co-cultured with PR1-CTL or 8F4 antibody. Cytotoxicity to PR1-targeting immunotherapies will be measured in vitro using calcein-AM cytotoxicity assays. The therapeutic ability of $8 \mathrm{~F} 4$ antibody to eliminate human multiple myeloma in vivo will be investigated using a human multiple myeloma xenograft mouse model. Human U266 multiple myeloma cells will be transplanted into NSG mice. Mice will be treated with PR1-CTL or 8F4 antibody. Bioluminescent imaging and ELISA will be used to measure disease progression and assess the therapeutic potential of $8 \mathrm{~F} 4$ in multiple myeloma. Additionally, we will investigate how $8 \mathrm{~F} 4$ can be integrated into standard of care therapies for multiple myeloma. Xenograft mice will be treated with 8F4 in combination with bortezomib, a standard of care multiple myeloma chemotherapy, to determine whether efficacy can be further increased with a combinatorial therapy approach. 


\section{Chapter 4: Determine the extent of NE and P3 uptake and}

\section{PR1 cross-presentation by multiple myeloma}

The following chapter is based on my publication, used with permission granted by AACR Journals.

Alatrash, G. ${ }^{1}$, Perakis, A.A. ${ }^{1}$, Kerros, C., Peters, H.L., Sukhumalchandra, P., Zhang, M., Jakher, H., Zope, M., Patenia, R., Sergeeva, A., Yi, S., Young, K.H., Philips, A.V., Cernosek, A.M., Garber, H.R., Qiao, N., Weng, J., St John, L.S., Lu, S., Clise-Dwyer, K., Mittendorf, E.A., Ma, Q. \& Molldrem, J.J. Targeting the Leukemia Antigen PR1 with Immunotherapy for the Treatment of Multiple Myeloma. Clin Cancer Res 24, 3386-3396 (2018).

\subsection{Multiple myeloma cells lacking endogenous NE and P3 internalize exogenous proteins}

To determine if multiple myeloma cells express NE and P3, a panel of multiple myeloma cell lines was analyzed for endogenous expression of these PGP. Our data indicate that NE and P3 are absent in multiple myeloma cells at the transcript and protein levels (Figure $2 A$ and $B$ ). These findings are in agreement with reports from the Cancer Cell Line Encyclopedia, ${ }^{59}$ which confirm the lack of NE and P3 in multiple myeloma (Figure 3A and B). The absence of NE and P3 in multiple myeloma is expected since multiple myeloma is of lymphoid origin, which is known to be deficient in myeloid primary granule proteins. ${ }^{60}$ The myelomonocytic U937 leukemia cell line that endogenously expresses NE and P3 was used as a positive control in these experiments. 
Figure 2. Multiple myeloma cells lack endogenous NE and P3.

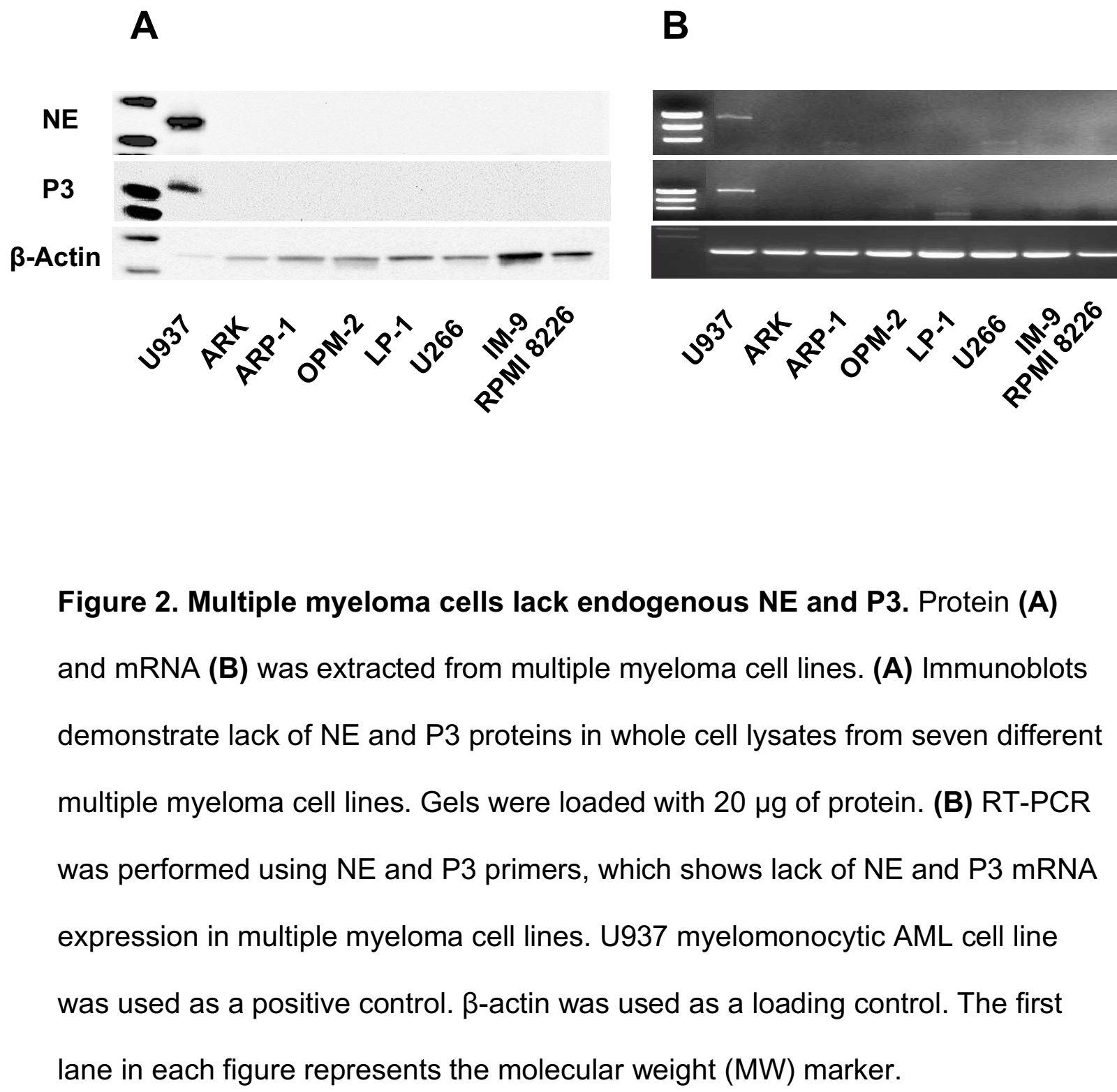


Figure 3. Transcript expression of NE and P3 genes in Cancer Cell Line Encyclopedia (CCLE).

A

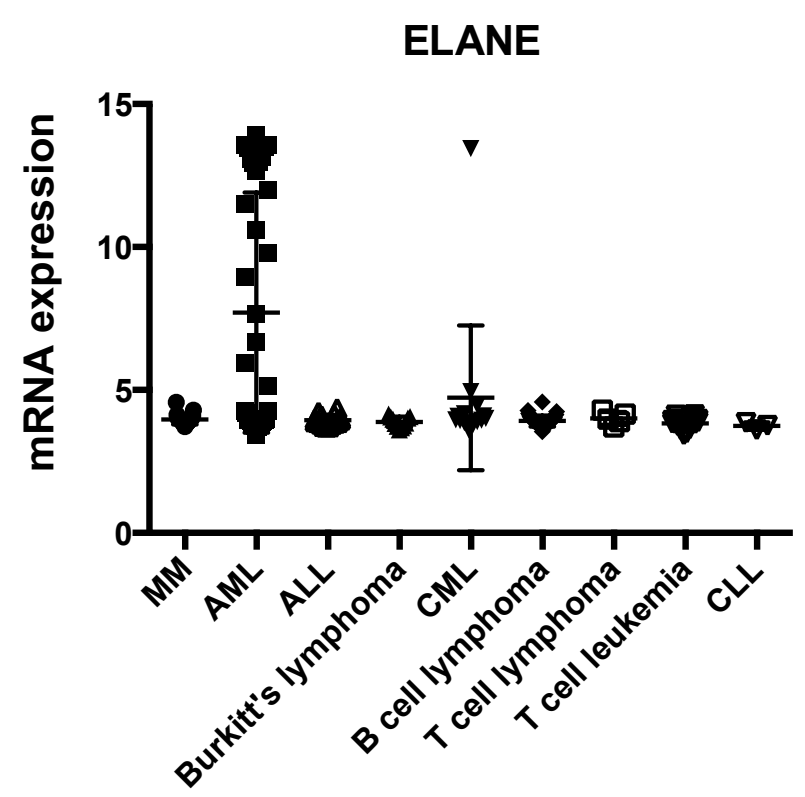

B

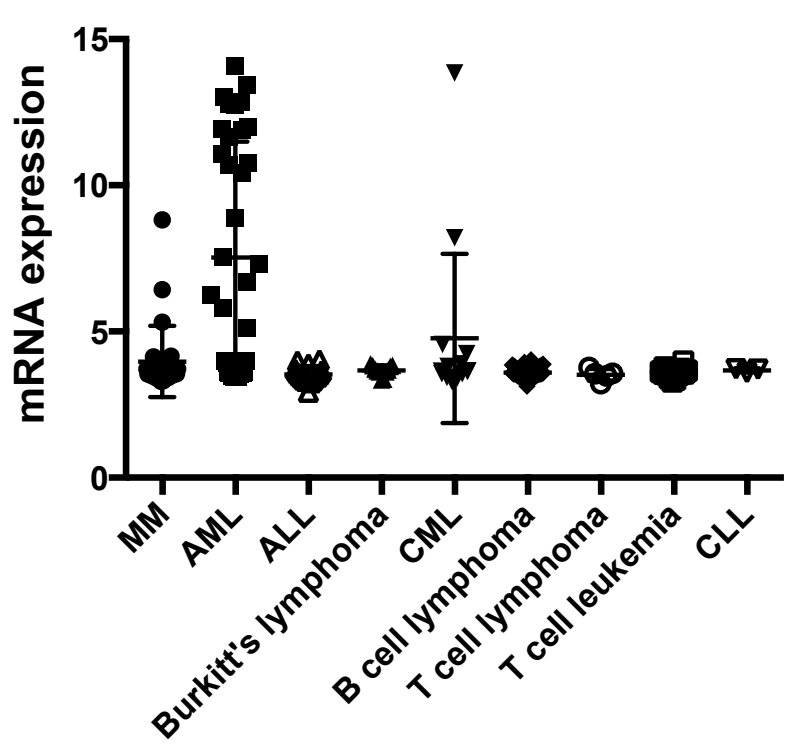

Figure 3. Transcript expression of NE and P3 genes in Cancer Cell Line Encyclopedia (CCLE). Transcript expression of ELANE (A) and PRNT3 (B) from cell lines was accessed from the CCLE for the following hematological malignancies: multiple myeloma, (MM, 25), acute myeloid leukemia (AML, 36), acute lymphoblastic leukemia (ALL, 16), Burkett's lymphoma (11), chronic myelogenous leukemia (CML, 14), B cell lymphoma (42), T cell lymphoma (5), T cell leukemia (16), chronic lymphocytic leukemia (CLL, 3). Parentheses indicate the number of cell lines. 


\subsection{HLA-A2 ${ }^{+}$multiple myeloma cells take up and cross-present NE and P3 from soluble and cell-associated sources}

Since we previously showed that solid tumors and B cells take up NE and $\mathrm{P} 3,40,41,43,53,61,62$ we next tested whether multiple myeloma cells take up NE and P3, the first step in antigen cross-presentation. We co-cultured a number of multiple myeloma cell lines with soluble NE or P3 over increasing durations. Flow cytometry analysis of intracellular NE and P3 staining demonstrates that NE and P3 are taken up by multiple myeloma (Figure 4A and B). Time-dependent internalization of NE and P3 is observed, with a marked difference in the magnitude of uptake between NE and P3. Our data indicates that soluble P3 is more efficiently internalized compared to NE by multiple myeloma cell lines. The uptake of both proteins is sustained throughout the duration of the cultures. The kinetics and degree of NE and P3 uptake by the multiple myeloma cell lines closely resembles the pattern of NE and P3 uptake observed in breast cancer and melanoma. 
Figure 4. Multiple myeloma cells internalize exogenous NE and P3.

A Intracellular NE

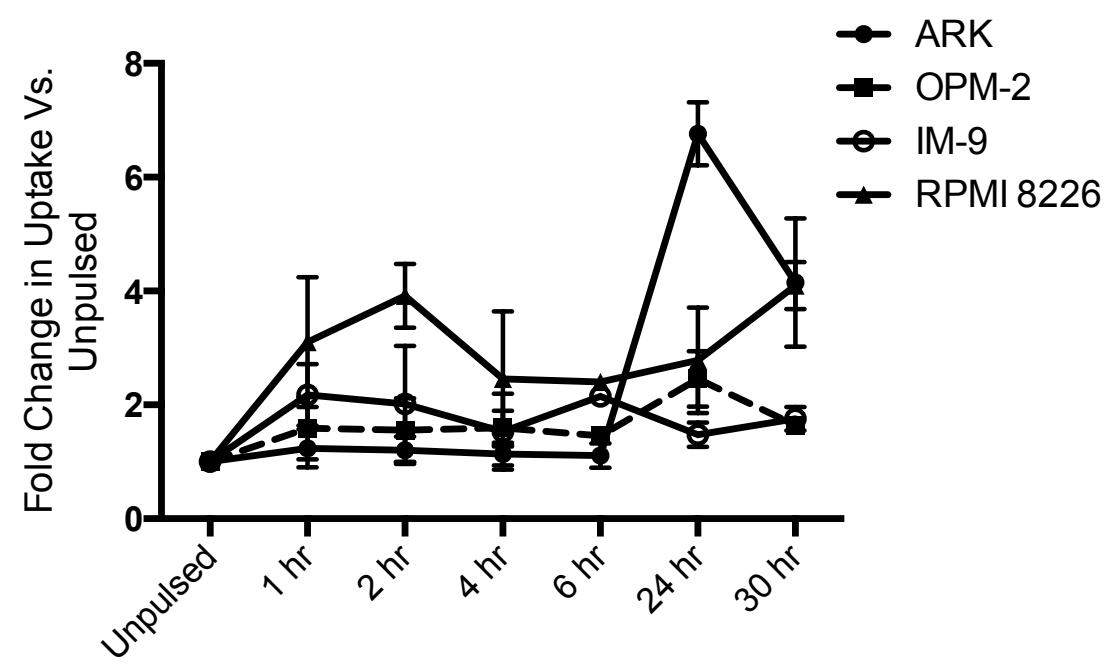

Time (hrs)

\section{B Intracellular P3}

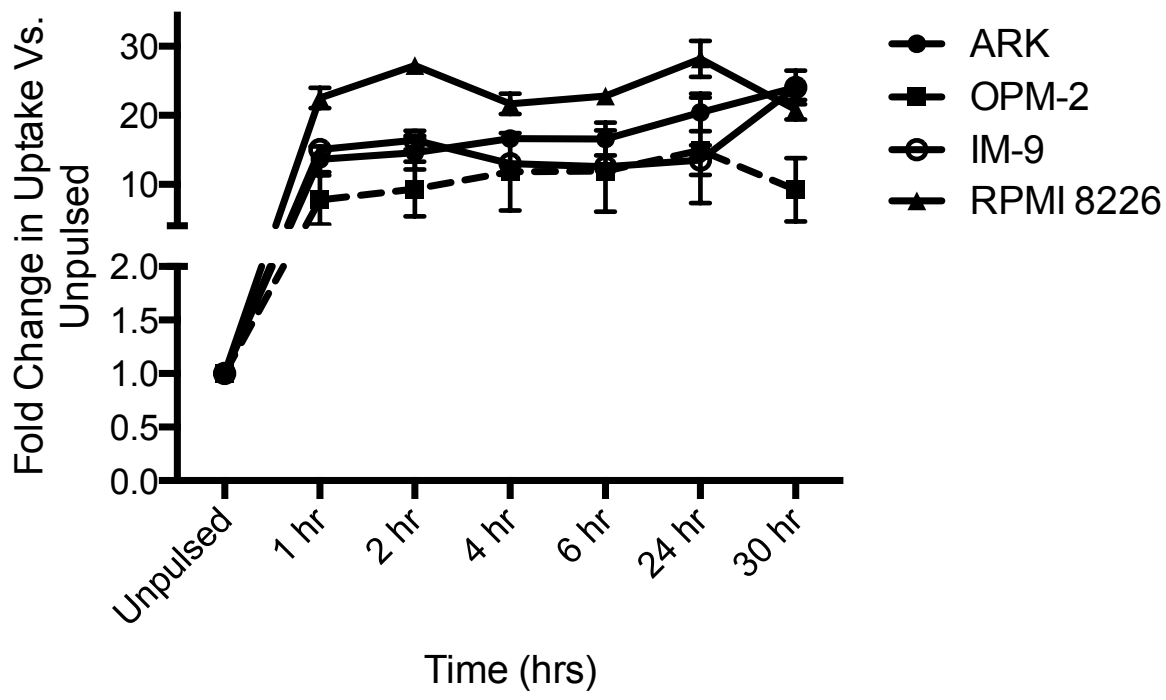


Figure 4. Multiple myeloma cells internalize exogenous NE and P3.

Flow cytometry detected intracellular NE and P3 in multiple myeloma cell lines following culture with NE $(10 \mu \mathrm{g} / \mathrm{mL})$ (A) or P3 $(10 \mu \mathrm{g} / \mathrm{mL})$ (B). After incubation, cells were permeabilized and stained with either anti-NE or anti-P3 antibodies. Graphs display the mean \pm SEM fold increase in median fluorescence intensity (MFI) over untreated cells from triplicate wells from six independent experiments. 
Since PR1 is an HLA-A2-restricted epitope, we focused our crosspresentation studies on the HLA-A2+ (i.e., HLA-A*0201) U266 multiple myeloma cell line. In agreement with the panel of multiple myeloma cell lines we examined (Figure 4A and B) we confirmed the internalization of soluble and PMNassociated NE and P3 by U266 cells (Figure 5A and B; Figure 6A and B). Previously, we demonstrated that solid tumor cells can cross-present PR1 from both soluble and cell-associated sources of NE and P3. ${ }^{40,43}$ These findings were recapitulated in the U266 multiple myeloma cells that were co-cultured with soluble NE, P3 or irradiated HLA-A2- PMN, the latter serving as the cellular source of NE and P3 that lack endogenous PR1 (Figure 7A and B).

As shown previously, cross-presentation was demonstrated by staining cells with anti-PR1/HLA-A2 Alexa-647-conjugated antibody. ${ }^{40}$ Due to the unique and inherent HLA-binding properties of TCR-like antibodies, we have observed low-affinity binding of $8 \mathrm{~F} 4$ to HLA-A2 in other cell lines, ${ }^{40,50}$ and also observed low background 8F4 staining in non-pulsed U266 cells. However, to highlight the specificity of $8 \mathrm{~F} 4$ for PR1/HLA-A2 complex, the HLA-A2- multiple myeloma cell lines, OPM-2 and RPMI 8226 were also co-cultured with HLA-A2- PMN and examined for cross-presentation (Figure 8). Thus, these data confirm NE and P3 internalization by $U 266$ multiple myeloma cells. Notably, there were differences in the magnitude of internalization between NE or P3 based on the protein source: PMN-derived NE achieved higher intracellular levels than soluble NE and was comparable to the uptake of PMN-derived P3. 
Figure 5. HLA-A2 ${ }^{+}$U266 cell line takes up NE and P3 from soluble and cellassociated sources.

\section{A Soluble NE and P3}

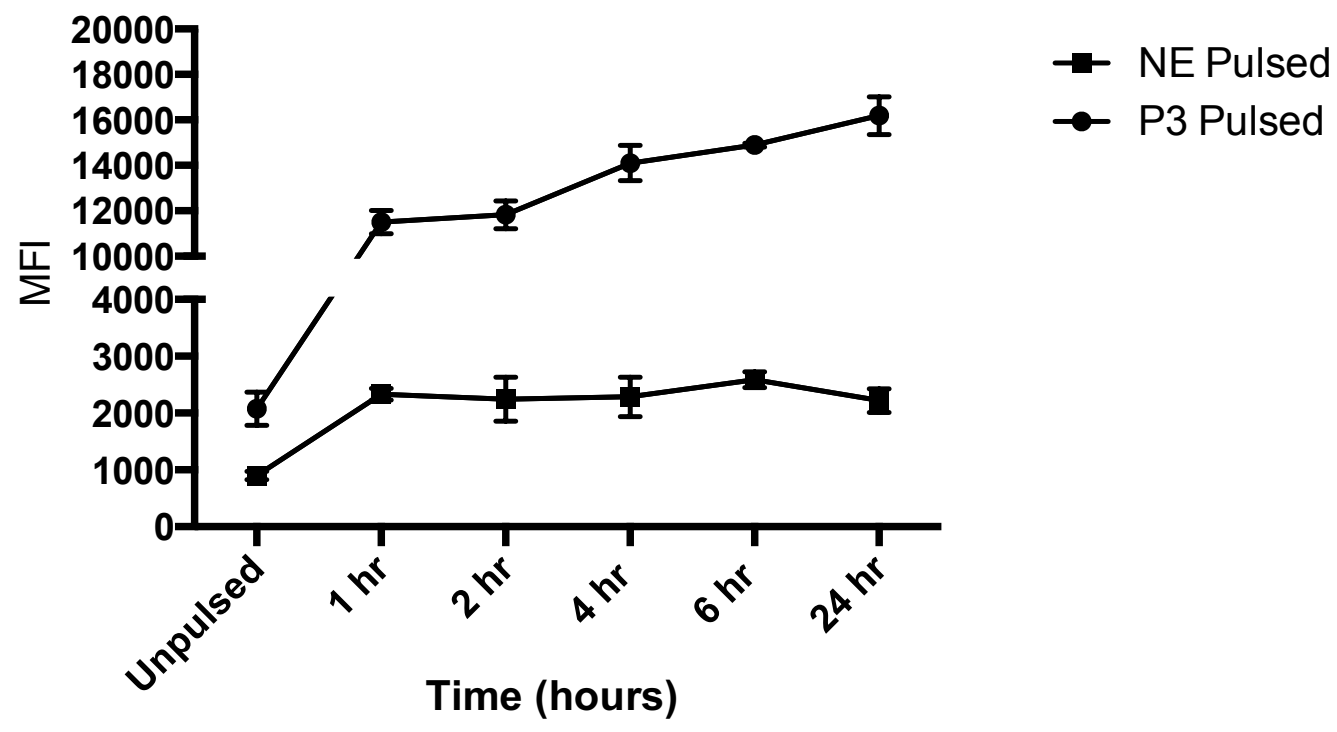

B Cell-Associated NE and P3

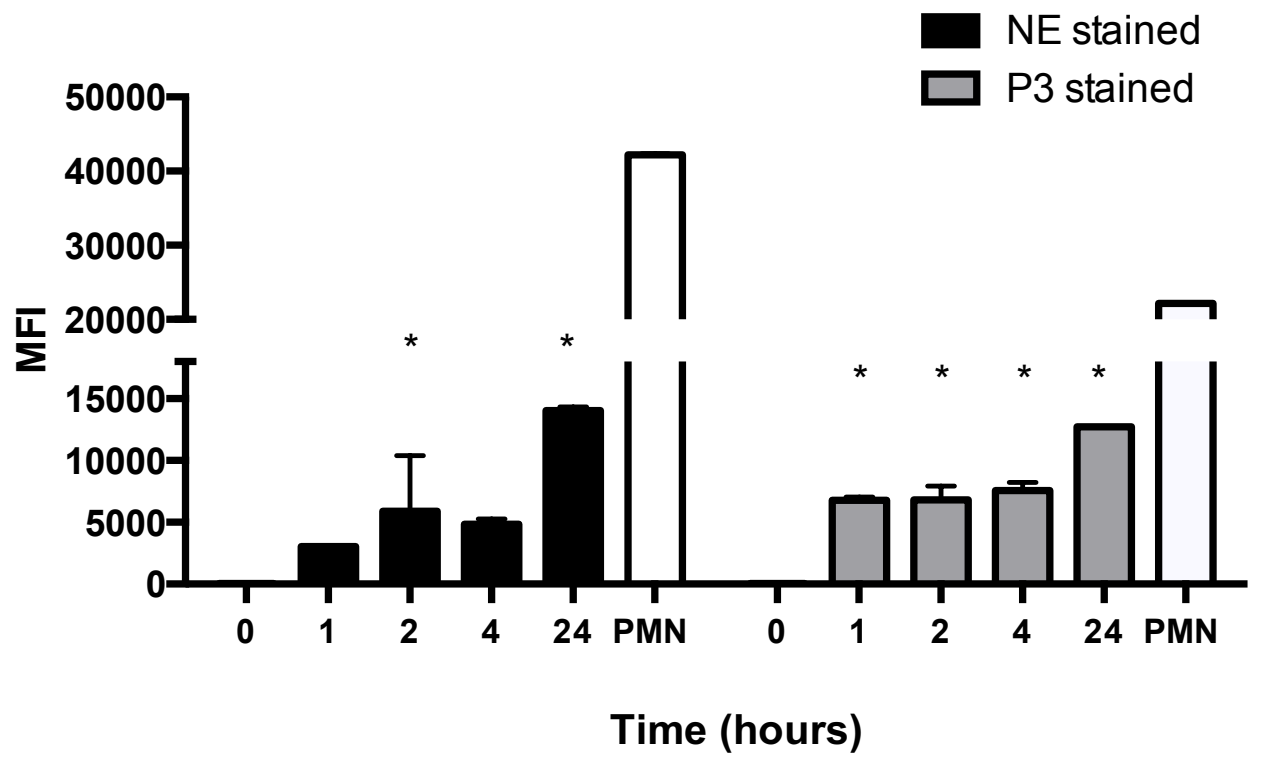


Figure 5. HLA-A2 ${ }^{+}$U266 cell line takes up NE and P3 from soluble and cellassociated sources. HLA-A2 ${ }^{+}$U266 multiple myeloma cell line was cultured with soluble NE $(10 \mu \mathrm{g} / \mathrm{mL})$ or P3 $(10 \mu \mathrm{g} / \mathrm{mL})(\mathbf{A})$, or irradiated HLA-A2- PMN (B) at the indicated time points. Cells were permeabilized, stained with anti-NE or anti-P3 antibody and analyzed by flow cytometry. For cell-associated uptake, light scatter and HLA-A2 staining seen on flow cytometry provided a clear distinction between PMN and U266 cells. PMN alone were used as a positive control for NE and P3 (white bars). ANOVA was performed using Prism 6.0 software $\left({ }^{*} P<0.05\right)$. Data are means \pm SEM from triplicate experiments. 
Figure 6. NE and P3 localize within the cell following co-culture with U266 multiple cells.

A

NE

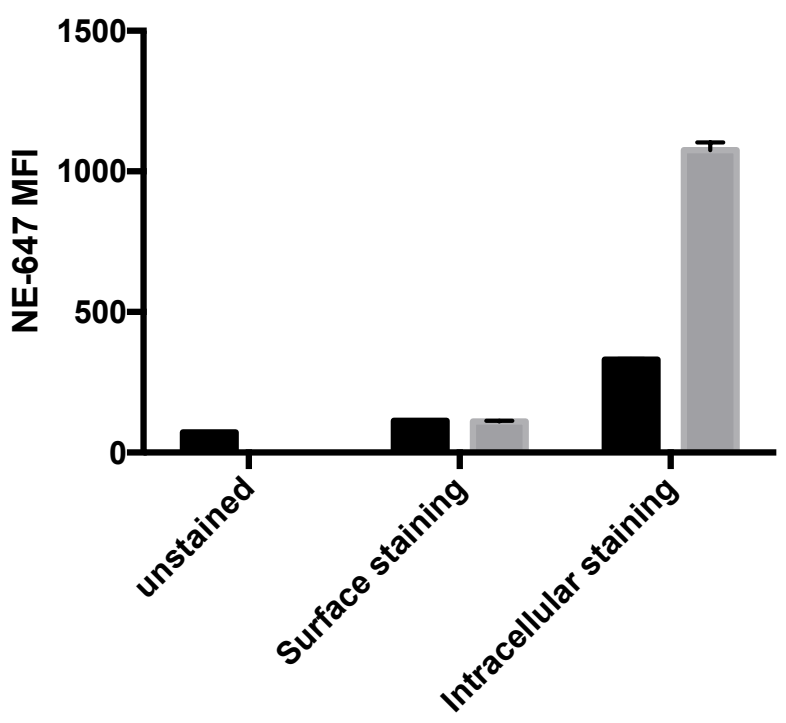

B

P3

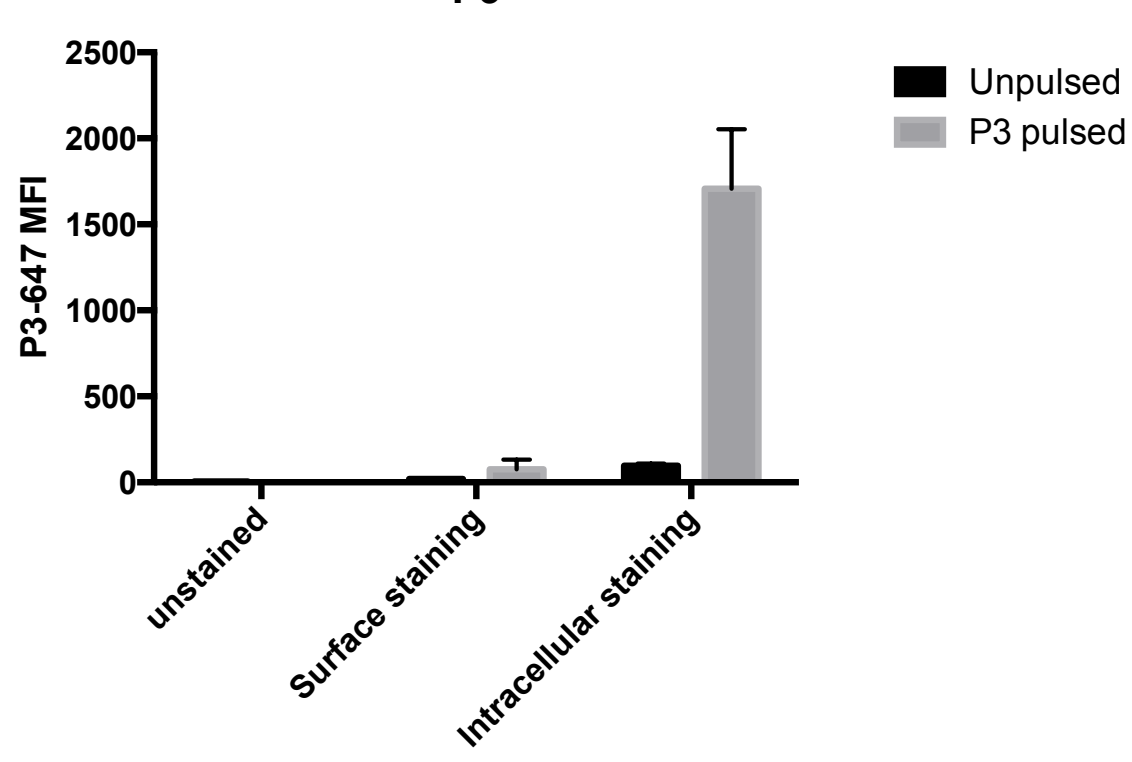

Unpulsed

P3 pulsed

Unpulsed

NE pulsed

B 
Figure 6. NE and P3 localize within the cell following co-culture with U266 multiple cells. HLA-A2+ U266 multiple myeloma cell line was cultured with soluble NE $(10 \mu \mathrm{g} / \mathrm{mL})(A)$ or P3 $(10 \mu \mathrm{g} / \mathrm{mL})$ (B). Cells were either surface stained or were permeabilized and then stained with anti-NE or anti-P3 antibody and analyzed by flow cytometry to confirm uptake. 
Figure 7. HLA-A2+ U266 cell cross-presents NE and P3 from soluble and cell-associated sources.

\section{A Soluble NE and P3}

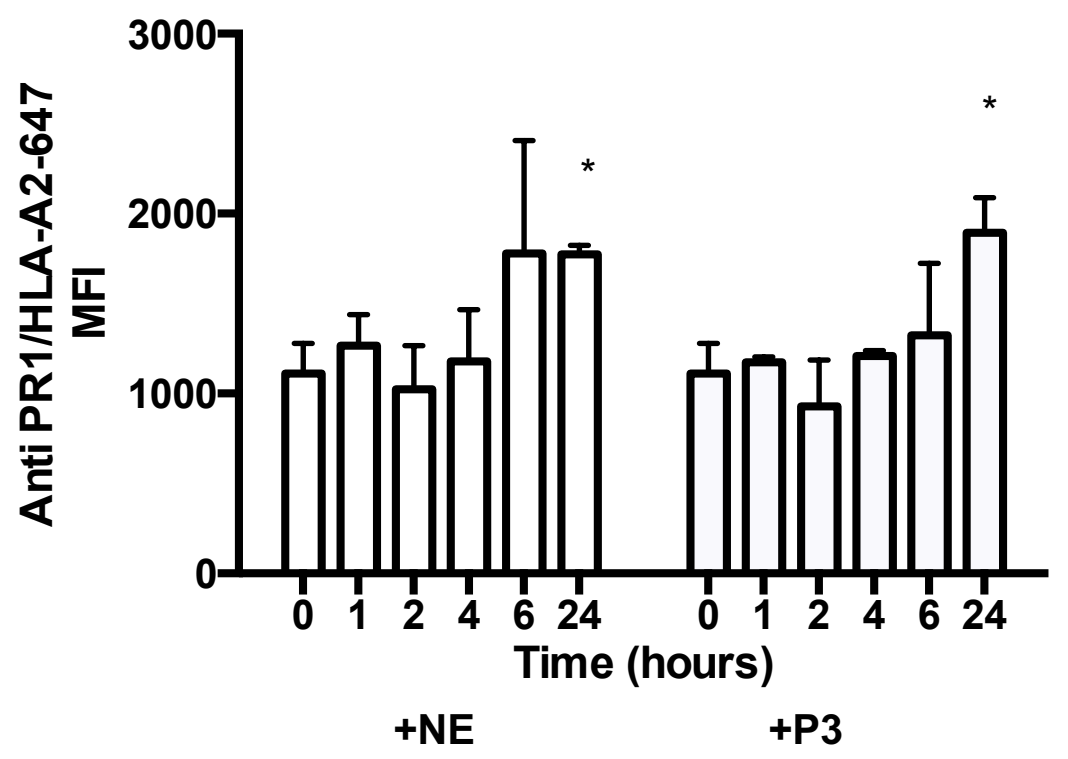

B Cell-Associated NE and P3

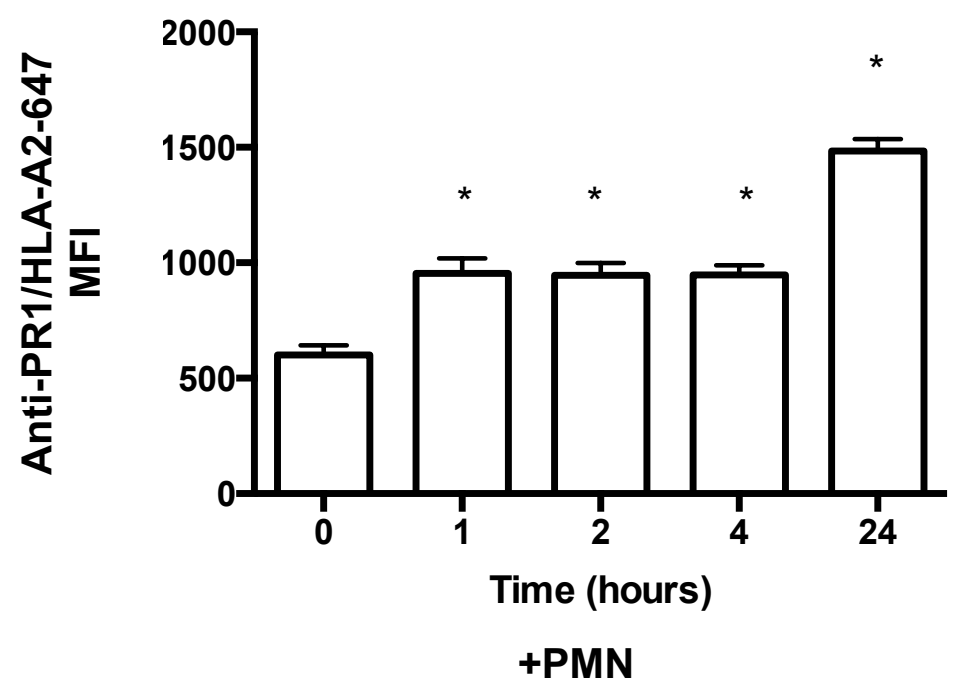


Figure 7. HLA-A2 ${ }^{+}$U266 cell cross-presents NE and P3 from soluble and cell-associated sources. U266 cells were cultured with soluble NE or P3 (10 $\mu \mathrm{g} / \mathrm{mL}$ ) (A) or irradiated PMN (B) then analyzed for expression of surface PR1/HLA-A2 using 8F4-Alexa 647. Mean \pm SEM fold increase of the median fluorescence intensity (MFI) of PR1/HLA-A2 is shown from duplicate experiments. Time "0" indicates untreated cells. ANOVA test was performed using Prism 6.0 software $\left({ }^{*} P<0.05\right)$. 
Figure 8. Absence of PR1 cross-presentation in HLA-A2 negative multiple myeloma cell lines.

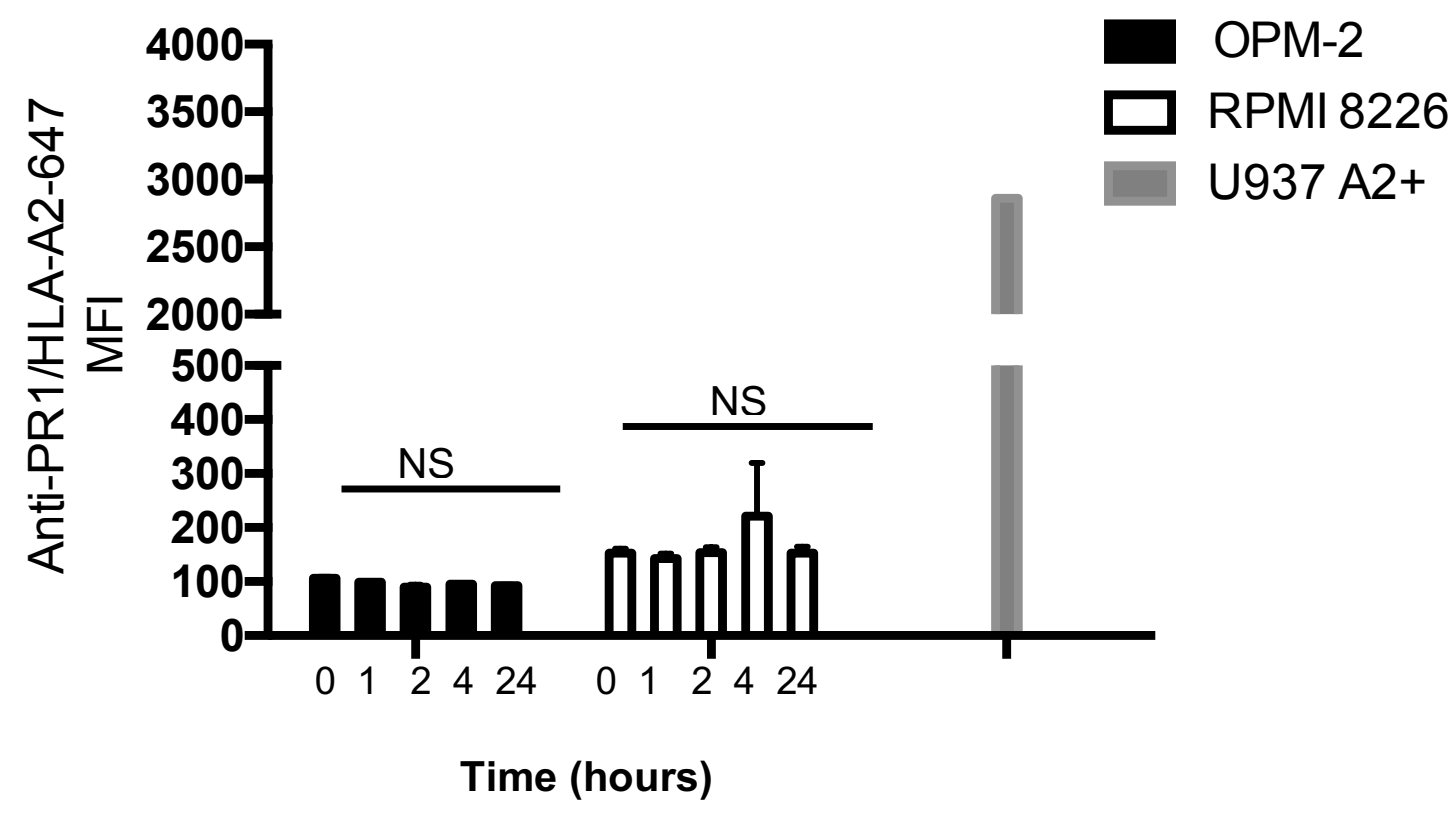

Figure 8. Absence of PR1 cross-presentation in HLA-A2 negative multiple myeloma cell lines. HLA-A2 negative OPM-2 and RPMI 8226 multiple myeloma cell lines were cultured with irradiated HLA-A2 negative PMN at the indicated time points then analyzed for expression of surface PR1/HLA-A2 using 8F4-Alex 647. Mean \pm SEM of the median fluorescence intensity (MFI) of PR1/HLA-A2 is shown from a representative experiment that was performed in duplicate. Time " 0 " indicates untreated cells. U937-A2 ${ }^{+}$cell line was used as a positive control for PR1/HLA-A2. ${ }^{50}$ ANOVA test was performed using Prism 6.0 software. NS, not significant. 


\subsection{Cross-presentation by multiple myeloma involves the proteasome and Golgi/ER.}

Cross-presentation involves distinct, well-defined pathways that utilize the proteasome and ER/Golgi. ${ }^{63-65}$ The proteasome plays an important role in antigen cross-presentation by cleaving intracellular proteins into small, 8-11 amino acid peptides, which are transported into the ER by the TAP1/2 complex. In the ER, peptides are loaded onto HLA class I molecules using components of the antigen processing machinery and are then exported to the cell surface via the Golgi. We hypothesized that NE and P3 cross-presentation involves proteasome and ER/Golgi shuttling, as previously shown for NE and P3 crosspresentation by solid tumors and APCs. ${ }^{40,41}$ Our data confirm that multiple myeloma utilizes the ER/Golgi and proteasome for NE and P3 crosspresentation, since incubation of cells with lactacystin, a proteasome inhibitor, and brefeldin A, which inhibits ER to Golgi antegrade transport, both decreased PR1/HLA-A2 expression (Figure 9A and B). 
Figure 9. Proteasome and ER/Golgi are involved in the cross-presentation of NE and P3 by U266 multiple myeloma cells.
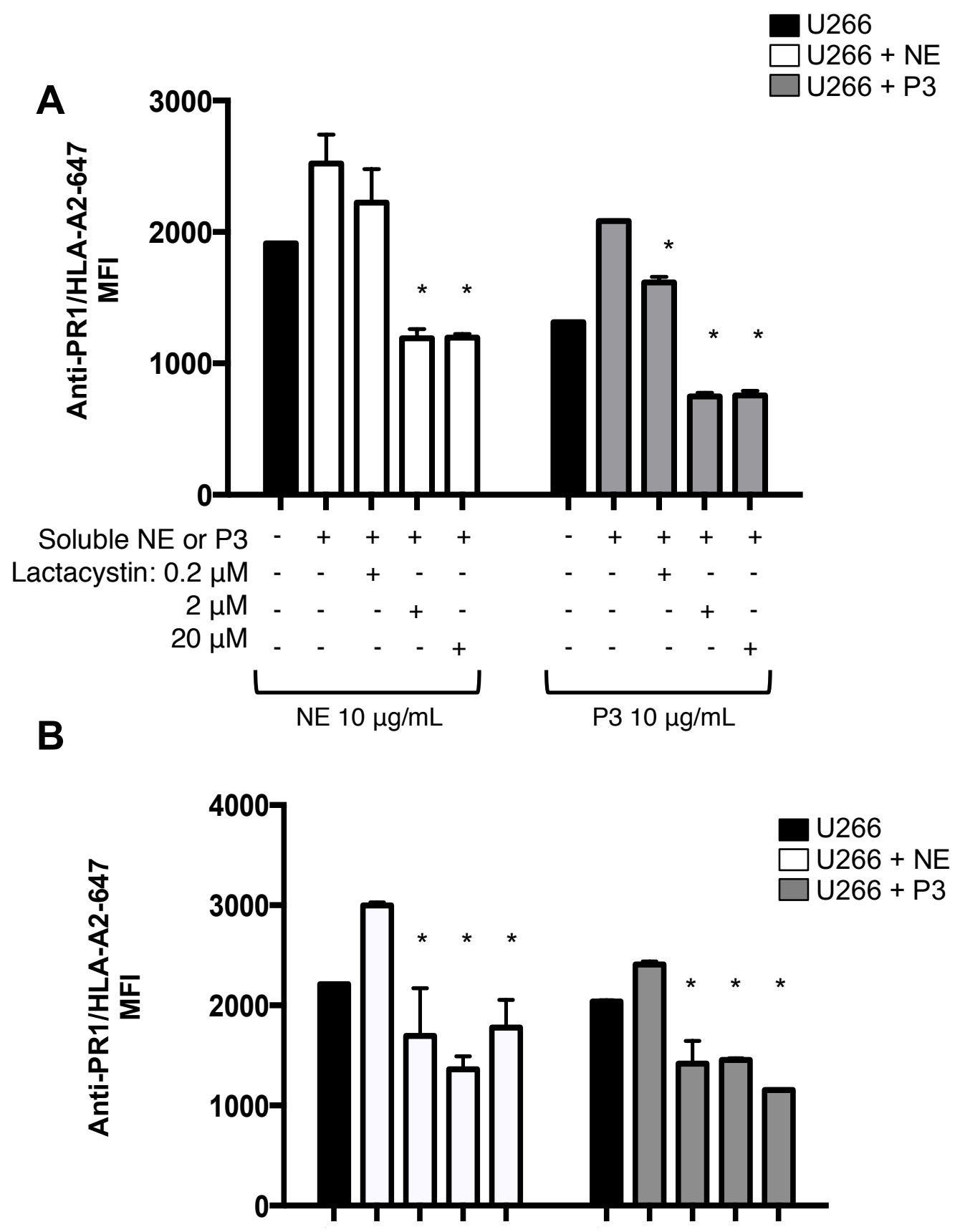

Soluble NE or P3 - $++++\quad-\quad++++$

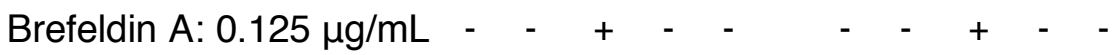

$1.25 \mu \mathrm{g} / \mathrm{mL} \quad-\quad-\quad-\quad+-\quad-\quad-\quad-+$

$2.5 \mu \mathrm{g} / \mathrm{mL} \quad-\quad-\quad-\quad-\quad+\quad-\quad-\quad-\quad-+$

NE $10 \mu \mathrm{g} / \mathrm{mL} \quad$ P3 $10 \mu \mathrm{g} / \mathrm{mL}$ 
Figure 9. Proteasome and ER/Golgi are involved in the cross-presentation of NE and P3 by U266 multiple myeloma cells. U266 multiple myeloma cells were cultured for 24 hours in media containing NE or P3 $(10 \mu \mathrm{g} / \mathrm{mL})$ and the antigen processing machinery inhibitors lactacystin (proteasome inhibitor) or brefeldin A (ER/Golgi transport inhibitor) (A, B). PBMC were cultured with U266 to serve as a negative control, since PBMC lack NE and P3. Cells were then analyzed using flow cytometry for expression of PR1/HLA-A2 after surface staining with 8F4-Alexa-647. Mean \pm SEM of the median fluorescence intensity (MFI) of PR1/HLA-A2 is shown from duplicate wells from 3 separate experiments. ANOVA test was performed using Prism 6.0 software $\left({ }^{*} P<0.05\right)$. 
Next, we translated our in vitro studies into a clinically relevant system by examining PMN as the source for NE and P3 and using bortezomib, a proteasome inhibitor approved for treatment of multiple myeloma. In agreement with our findings with lactacystin, bortezomib reduced PR1/HLA-A2 on the U266 myeloma cell surface (Figure 10). Additionally, the IMiD lenalidomide did not alter surface levels of PR1/HLA-A2 on U266 after co-culture with PMN (Figure 11). We also observed a consistent decrease in the surface expression of overall HLA-A2 due to inhibition of the proteasome after treatment with both lactacystin and bortezomib (Figure 12A and B). However, surface HLA-A2 expression was unaffected by brefeldin A or lenalidomide (Figure 13A and B). Since conventional antigen cross-presentation mechanisms employ proteasome,${ }^{37}$ it is not surprising that overall expression of HLA-A2 decreases with the use of lactacystin and bortezomib, and highlights the role of the proteasome in PR1 cross-presentation. 
Figure 10. Bortezomib reduces PR1/HLA-A2 expression on the cell surface of U266 multiple myeloma cells.

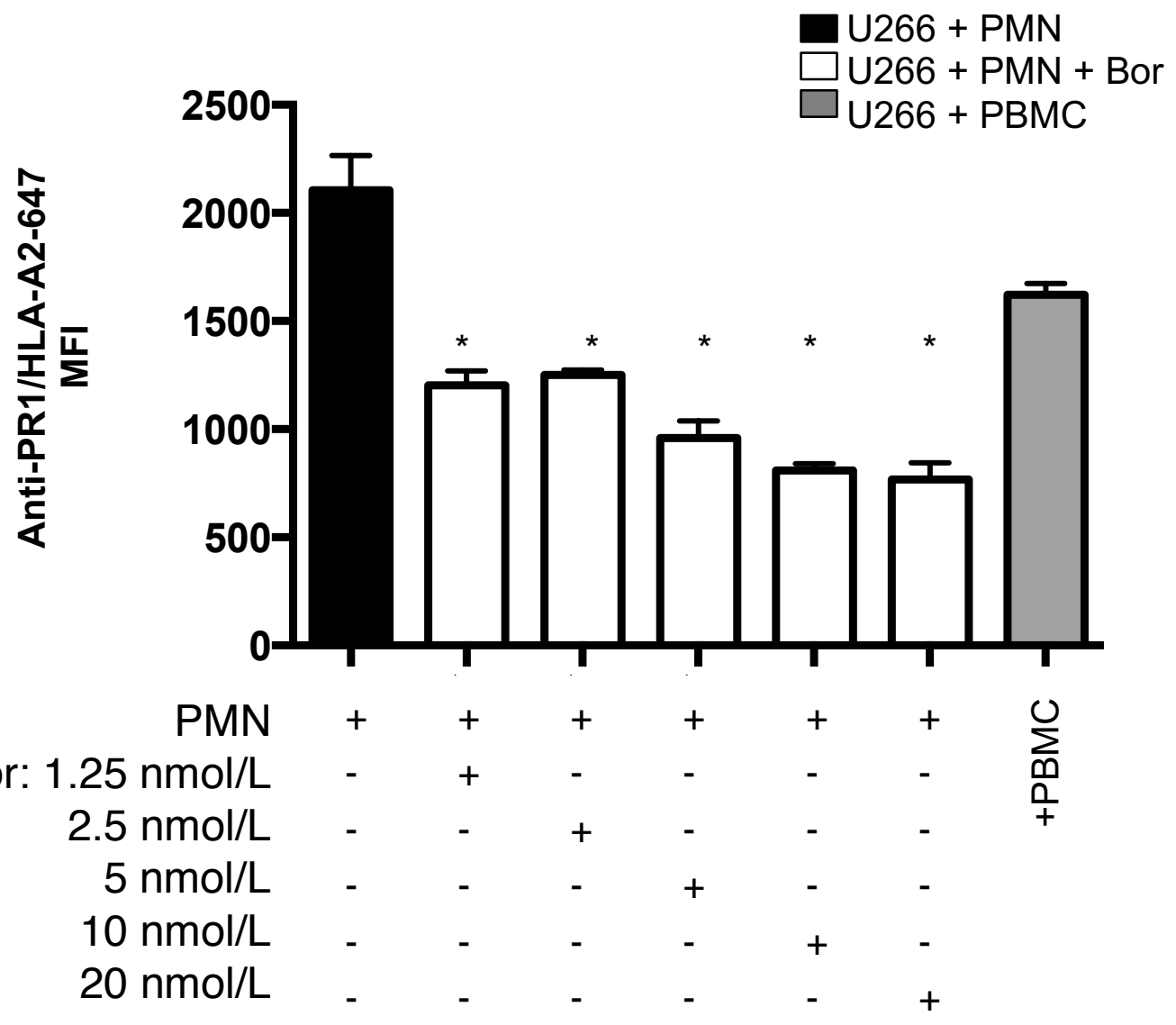


Figure 10. Bortezomib reduces PR1/HLA-A2 expression on the cell surface of U266 multiple myeloma cells. U266 multiple myeloma cells were cultured for 24 hours with PMN at a 1:1 U266:PMN ratio in media containing bortezomib (Bor). PBMC were cultured with U266 to serve as a negative control, since PBMC lack NE and P3. Cells were then analyzed using flow cytometry for expression of PR1/HLA-A2 after surface staining with 8F4-Alexa-647. Mean \pm SEM of the median fluorescence intensity (MFI) of PR1/HLA-A2 is shown from duplicate wells from 3 separate experiments. ANOVA test was performed using Prism 6.0 software $\left({ }^{*} P<0.05\right)$. 
Figure 11. Lenalidomide does not reduce PR1/HLA-A2 expression on the cell surface of U266 multiple myeloma cells.

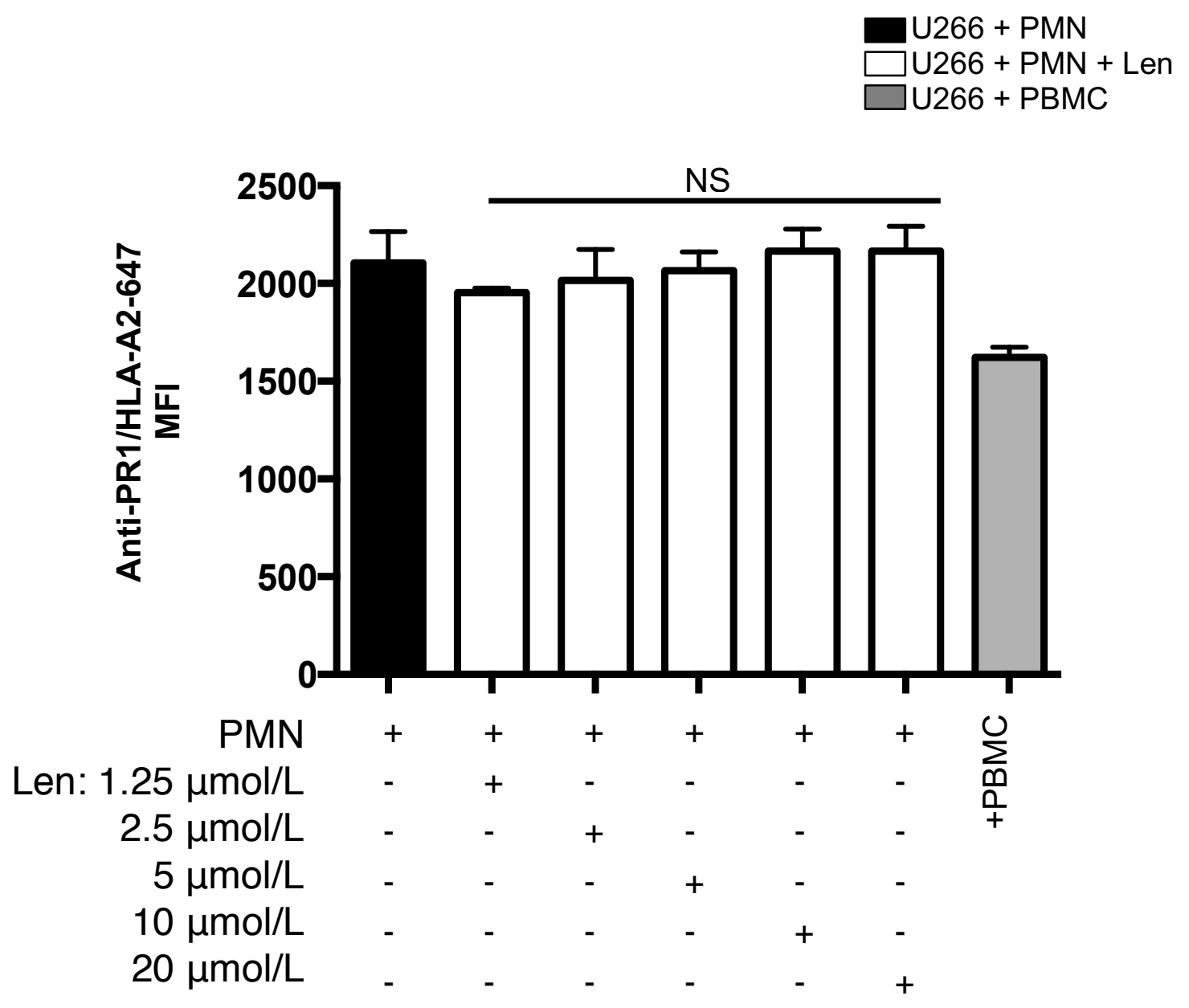


Figure 11. Lenalidomide does not reduce PR1/HLA-A2 expression on the cell surface of U266 multiple myeloma cells. U266 multiple myeloma cells were cultured for 24 hours with PMN at a 1:1 U266:PMN ratio in media containing lenalidomide (Len). PBMC were cultured with U266 to serve as a negative control, since PBMC lack NE and P3. Cells were then analyzed using flow cytometry for expression of PR1/HLA-A2 after surface staining with 8F4Alexa-647. Mean \pm SEM of the median fluorescence intensity (MFI) of PR1/HLAA2 is shown from duplicate wells from 3 separate experiments. ANOVA test was performed using Prism 6.0 software. NS, not significant. 
Figure 12. HLA-A2 expression on U266 is reduced after addition of proteasome inhibitors.

A

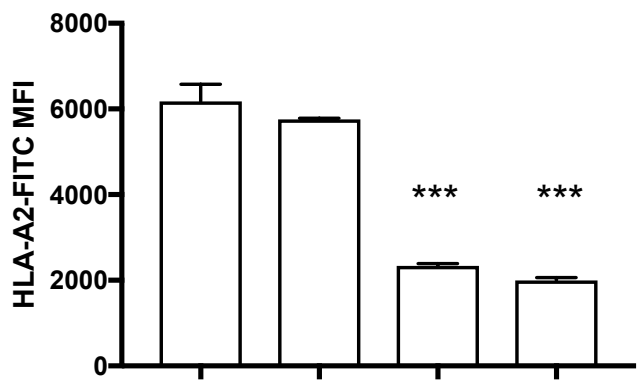

Soluble NE or P3 Lactacystin: $0.2 \mu \mathrm{M}$ $2 \mu \mathrm{M}$ $20 \mu \mathrm{M}$

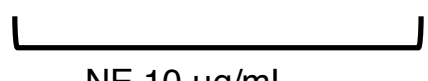

$\mathrm{NE} 10 \mu \mathrm{g} / \mathrm{mL}$

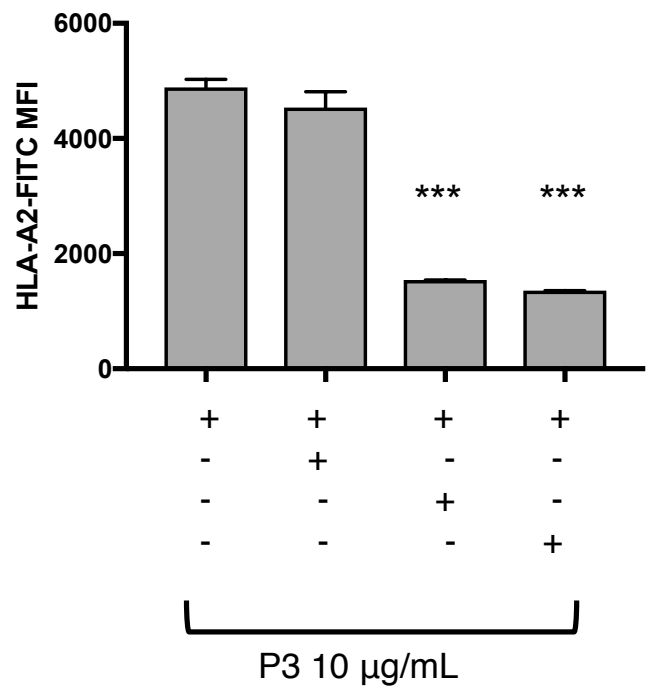

P3 $10 \mu \mathrm{g} / \mathrm{mL}$

B

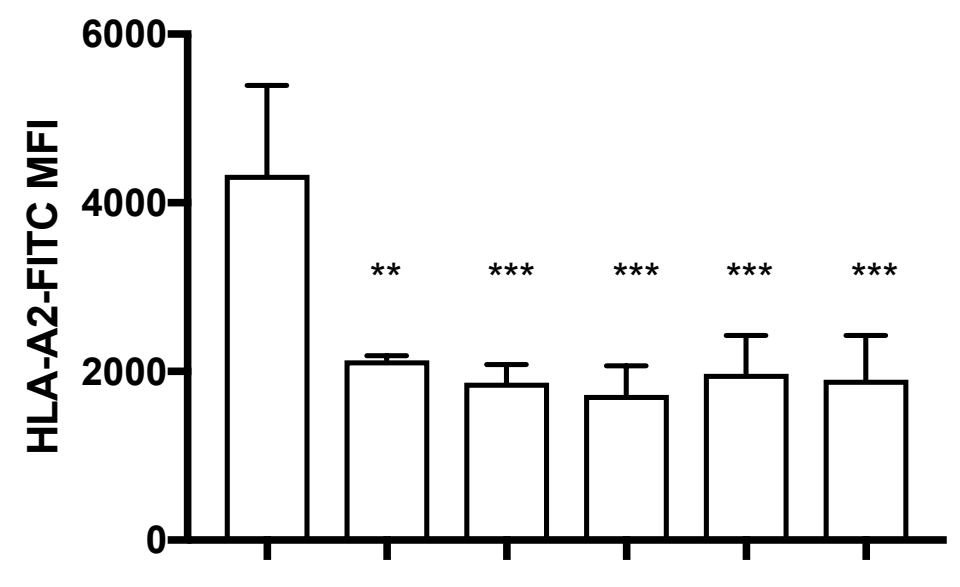

Bor: $1.25 \mathrm{nmol} / \mathrm{L}$

$2.5 \mathrm{nmol} / \mathrm{L}$

$5 \mathrm{nmol} / \mathrm{L}$

$10 \mathrm{nmol} / \mathrm{L}$

$20 \mathrm{nmol} / \mathrm{L}$ 
Figure 12. HLA-A2 expression on U266 is reduced after addition of proteasome inhibitors. U266 multiple myeloma cells were cultured for 24 hours in media containing NE or P3 $(10 \mu \mathrm{g} / \mathrm{mL})$ and lactacystin (proteasome inhibitor) (A) or were cultured for 24 hours with PMN at a 1:1 U266:PMN ratio in media containing bortezomib (Bor) (B). Cells were then analyzed using flow cytometry for expression of HLA-A2. Mean \pm SEM of the median fluorescence intensity (MFI) of HLA-A2 is shown from duplicate wells from 3 separate experiments. ANOVA test was performed using Prism 6.0 software $\left({ }^{* *} P<0.01\right.$, $\left.{ }^{* * *} P<0.001\right)$. 
Figure 13. HLA-A2 expression on U266 is not reduced after addition of brefeldin A or lenalidomide.

A

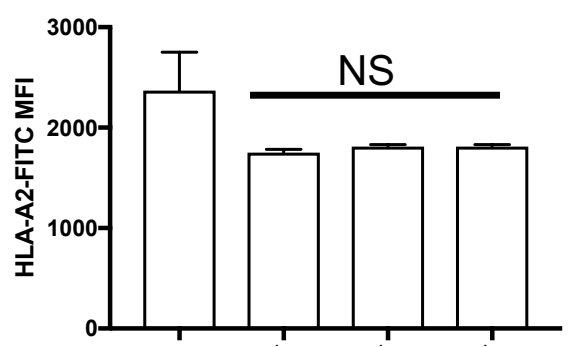

Soluble NE or P3 + Brefeldin A: $0.125 \mu \mathrm{g} / \mathrm{mL}$

$1.25 \mu \mathrm{g} / \mathrm{mL}$

$2.5 \mu \mathrm{g} / \mathrm{mL}$
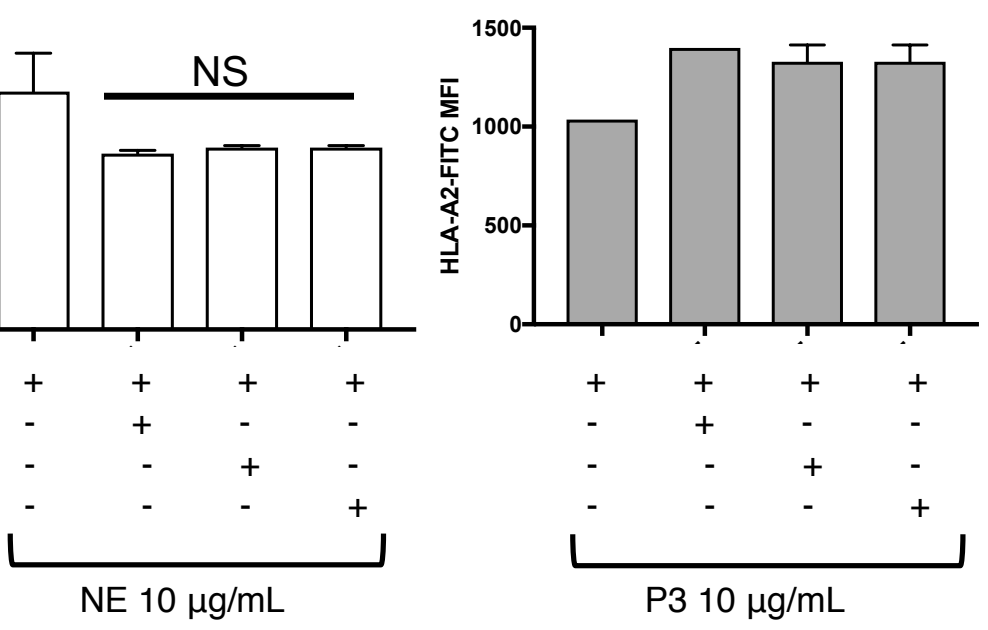

B

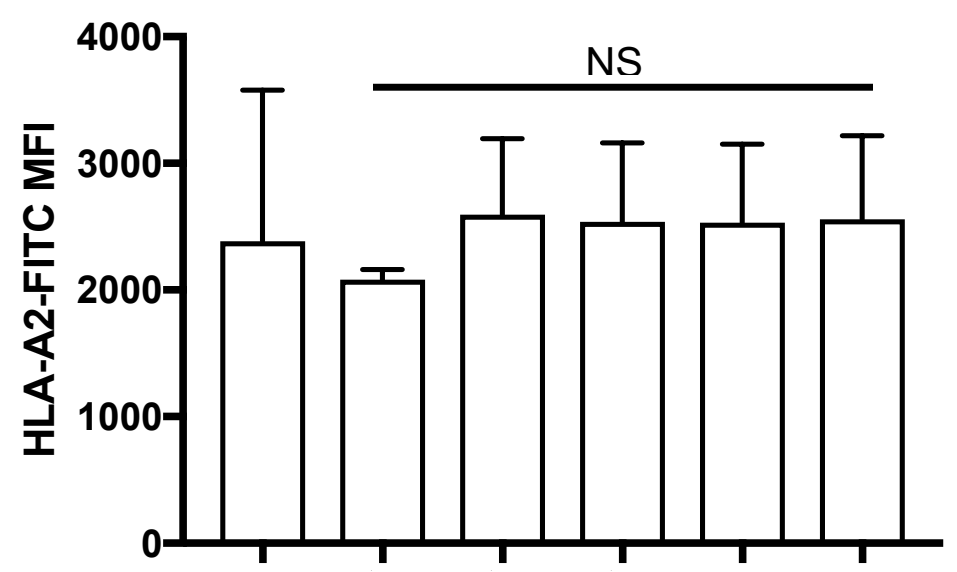

Len: $1.25 \mu \mathrm{mol} / \mathrm{L}$

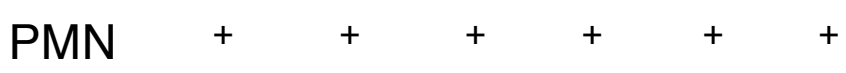

$2.5 \mu \mathrm{mol} / \mathrm{L}$

$5 \mu \mathrm{mol} / \mathrm{L}$

$10 \mu \mathrm{mol} / \mathrm{L}$

$20 \mu \mathrm{mol} / \mathrm{L}$ 
Figure 13. HLA-A2 expression on U266 is not reduced after addition of brefeldin A or lenalidomide. U266 multiple myeloma cells were cultured for 24 hours in media containing NE or P3 $(10 \mu \mathrm{g} / \mathrm{mL})$ and brefeldin A (ER/Golgi transport inhibitor) (A) or were cultured for 24 hours with PMN at a 1:1 U266:PMN ratio in media containing lenalidomide (Len) (B). Cells were then analyzed using flow cytometry for expression of HLA-A2. Mean \pm SEM of the median fluorescence intensity (MFI) of HLA-A2 is shown from duplicate wells from 3 separate experiments. ANOVA test was performed using Prism 6.0 software. NS, not significant. 


\subsection{PR1 cross-presentation by multiple myeloma is TAP-dependent}

Since we previously demonstrated that PR1 cross-presentation utilizes the proteasome and ER/Golgi, conventional components of antigen presentation, we further investigated the role of the other antigen presentation machinery proteins (APM). As discussed earlier in this work, cross-presentation can utilize TAP to transport peptides from the cytosol into the ER for HLA loading. However, there also are other described peptide loading mechanisms that are TAP-independent. To characterize the role of TAP in multiple myeloma cross-presentation, we knocked-down TAP expression in U266 cells by siRNA. si-TAP U266 cells were then cultured in media supplemented with soluble NE to facilitate crosspresentation. Calcein-AM cytotoxicity assays show significant reduction in PR1CTL recognition at 5:1 E:T ratios. This indicates that si-TAP U266 cells pulsed with NE were less susceptible to killing by PR1-CTL than si-control U266 cells (Figure 14A). These data suggest that TAP is required for cross-presentation of NE and PR1/HLA-A2 expression by U266. Sufficient TAP knock-down in U266 was confirmed by western blot (Figure 14B). Additionally, the total HLA expression was unaffected by siRNA further suggesting these observations are not due to global change in peptide HLA expression (Figure 14B). 
Figure 14. TAP knock-down in U266 results in decreased PR1/HLA-A2 expression and PR1-mediated killing.

A

$\rightarrow$ si-control U266+NE

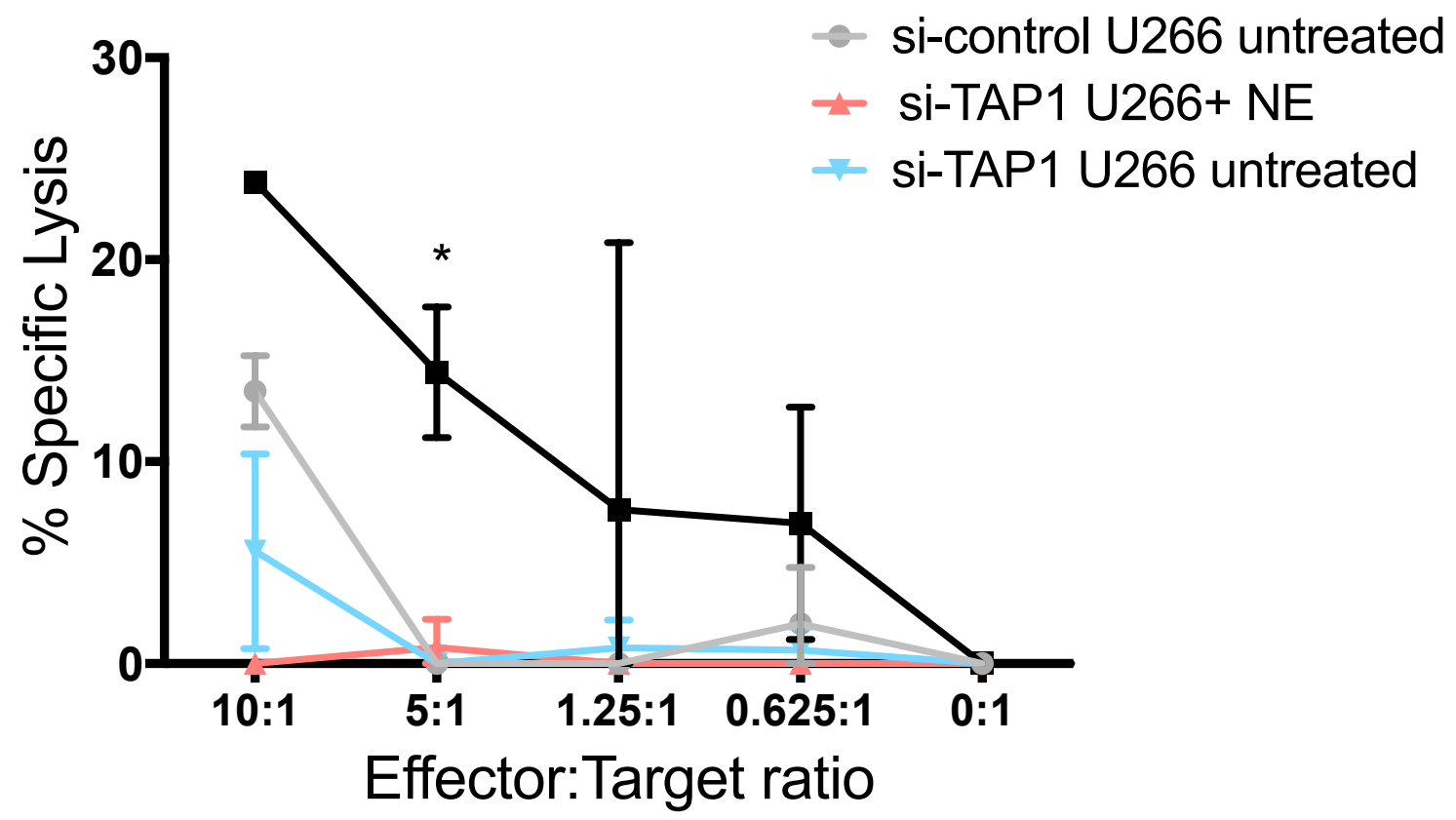

B

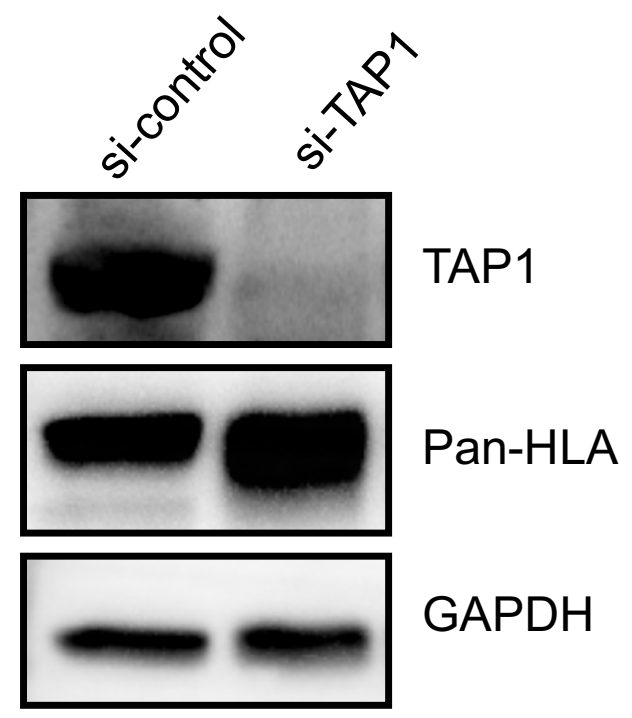


Figure 14. TAP knock-down in U266 results in decreased PR1/HLA-A2 expression and PR1-mediated killing. (A) TAP1 was knocked-down in U266 cells using siRNA for TAP1. After 48 hours, U266 cells we cultured with or without soluble NE $(10 \mu \mathrm{g} / \mathrm{mL})$ supplemented media for 24 hours. Calcein AM cytotoxicity assays using PR1-specific CTL were then performed at varying effetor:target (E:T) ratios. Significantly higher killing of target cells was seen at the 5:1 E:T ratios for the si-control U266 +NE group compared to both si-TAP1 U266+NE and si-control U266 untreated group. $\left({ }^{\star} P<0.05\right)$. Statistical analysis was performed using ANOVA test. (B) Western blot analysis confirms sufficient knock-down of TAP1 was achieved in U266 cells when treated with si-TAP1. GAPDH was used as a loading control. 
Additionally, we sought to investigate whether the other APM proteins were differentially expressed in multiple myeloma patients. To this end, we analyzed the expression of the core components of the APM: calnexin, TAP, tapasin, LMP2/7 in patient multiple myeloma cells using western blot (Figure 15). Expression among the APM proteins, LMP7, LMP2, and tapasin were shown to be upregulated in multiple myeloma patients when compared to healthy donor B cells. We did not observe protein expression differences in the other APM components, calnexin and HLA. However, we did observe a lower expressed isoform of calnexin in Patient 3 who also showed decreased HLA expression. We further investigated the expression of calnexin and HLA among 10 other multiple myeloma patients by western blot (Figure 16). Our data show that two prominent isoforms of calnexin are expressed in multiple myeloma patient cells and this is correlated with lower HLA expression in patients that express the lower molecular weight isoform of calnexin. Interestingly, the lower HLA expression pattern observed in these multiple myeloma patients resembled a similar doubleband phenotype of immature HLA molecules when retained in the ER. ${ }^{66}$ This expression pattern may suggest a potential dominant negative effect mediated through improper chaperone functions by an alternative calnexin isoform where immature HLA molecules are retained in the ER and prevented from proper folding and egress to the cell surface. 
Figure 15. APM proteins LMP7, LMP2, and tapasin are upregulated in multiple myeloma patients.

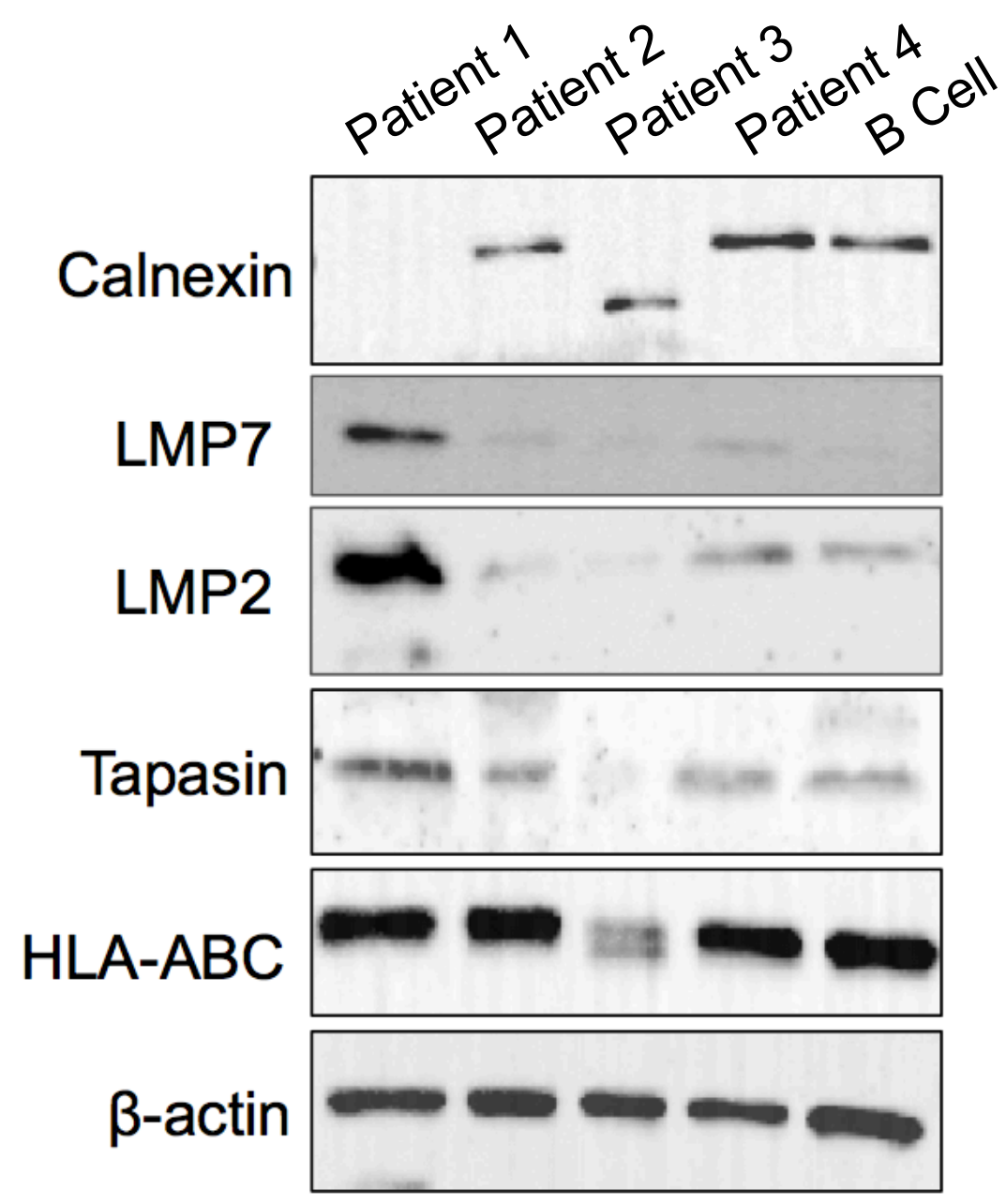


Figure 15. APM proteins LMP7, LMP2, and tapasin are upregulated in multiple myeloma patients. The expression of the major proteins that comprise the antigen presentation machinery (APM) were analyzed via western blotting. Whole cell lysates were generated from $\mathrm{CD} 138^{+}$enriched cells from the bone marrow of newly diagnosed multiple myeloma patients. B cells sorted from healthy donor peripheral blood monocytes were used as a control for nonmalignant protein expression levels. Patient whole cell lysates were loaded and separated by gel electrophoresis under reducing conditions. $\beta$-actin was used as a loading control. Expression among the APM proteins and across patients appears variable, with expression observed to be upregulated in some proteins (LMP7, LMP2, Tapasin) or unchanged (HLA, Calnexin). 
Figure 16. Multiple myeloma patients express different isoforms of calnexin.

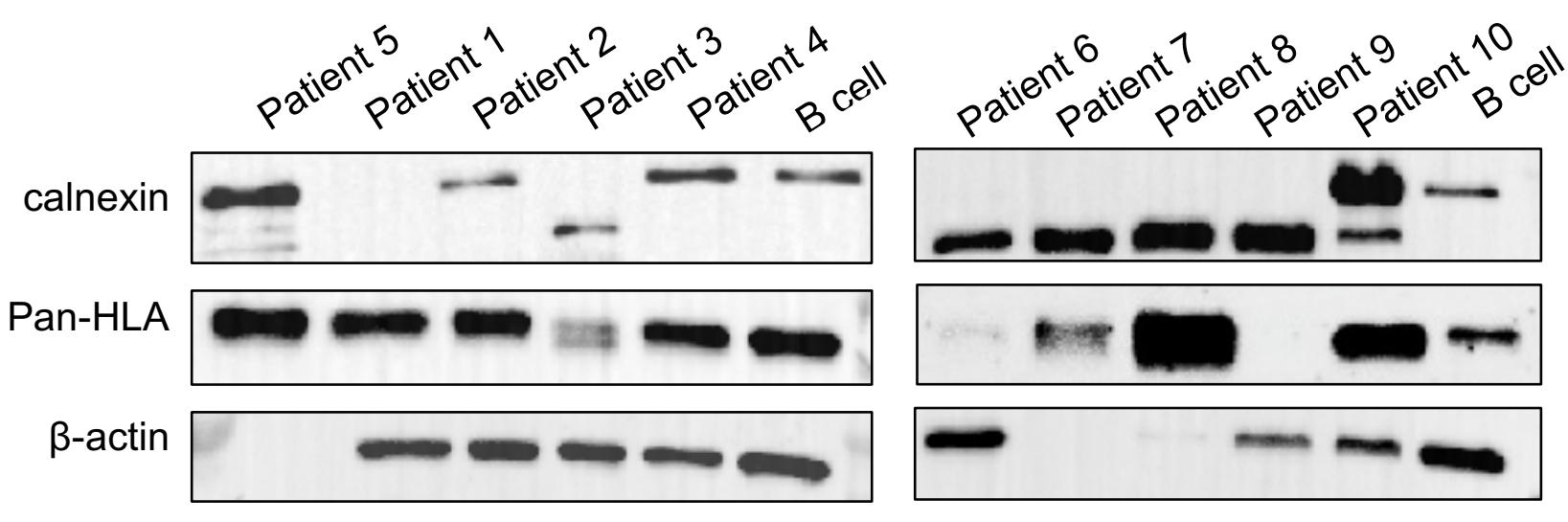

Figure 16. Multiple myeloma patients express different isoforms of calnexin. Whole cell protein lysates were generated from $\mathrm{CD} 138^{+}$cells of 10 newly diagnosed multiple myeloma patients as previously described in Figure 15. Western blotting was performed using antibodies against calnexin, HLA-ABC and $\beta$-actin as a loading control. Western blot analysis indicates two isoforms of calnexin expressed in multiple myeloma patients, with the lower molecular weight isoform correlating to a decreased expression of Pan-HLA. 


\subsection{Chapter 4 Summary}

Collectively our data demonstrates that multiple myeloma is capable of cross-presenting exogenous antigens. We have modeled this phenomenon by tracking the expression of two myeloid-restricted serine proteases, NE and P3. Multiple myeloma cells do not endogenously express NE or P3, but are capable of taking up these proteins from the extracellular environment and internalizing them in the cytoplasm. Furthermore, NE and P3 can be processed in a proteasome-dependent manner, leading to the expression of the PR1 antigen in the context of HLA-A2 on the multiple myeloma cell surface.

Importantly, multiple myeloma relies on the proteasome for effective cross-presentation of NE and $\mathrm{P} 3$, as use of proteasome inhibitors, lactacystin and bortezomib, muted the expression of PR1/HLA-A2 in U266 multiple myeloma cells. Additionally, use of Golgi transport inhibitor, brefeldin A, revealed that multiple myeloma cross-presentation also relies on the canonical ER to Golgi shuttling pathway for PR1/HLA-A2 surface expression. Our data also supports a TAP-dependent mechanism for PR1 cross-presentation as silencing TAP in U266 resulted in decreased PR1/HLA-A2 and PR1-CTL recognition. Treatment with the IMiD lenalidomide did not affect the ability of U266 cells to cross-present $\mathrm{NE}$ and $\mathrm{P} 3$ as there was no observed decrease in PR1/HLA-A2 expression.

Overall our data thus far have revealed a unique mechanism by which multiple myeloma cells can express a novel antigen, PR1, via cross-presentation. 


\section{Chapter 5: Evaluate the susceptibility of multiple myeloma to PR1-targeting immunotherapies}

The following chapter is based on my publication, used with permission granted by AACR Journals.

Alatrash, G. ${ }^{1}$, Perakis, A.A. ${ }^{1}$, Kerros, C., Peters, H.L., Sukhumalchandra, P., Zhang, M., Jakher, H., Zope, M., Patenia, R., Sergeeva, A., Yi, S., Young, K.H., Philips, A.V., Cernosek, A.M., Garber, H.R., Qiao, N., Weng, J., St John, L.S., Lu, S., Clise-Dwyer, K., Mittendorf, E.A., Ma, Q. \& Molldrem, J.J. Targeting the Leukemia Antigen PR1 with Immunotherapy for the Treatment of Multiple Myeloma. Clin Cancer Res 24, 3386-3396 (2018).

\subsection{PR1 cross-presentation increases the susceptibility of multiple myeloma to PR1-CTL and 8F4 antibody}

Immunotherapy targeting PR1 has shown promising efficacy in the treatment of myeloid malignancies. ${ }^{49,50,67,68}$ Thus, we investigated whether PR1 cross-presentation by multiple myeloma cells could lead to their lysis by PR1CTL and the complement-fixing, anti-PR1/HLA-A2 8F4 antibody. Calcein-AM cytotoxicity assays demonstrate that cross-presentation of NE and P3 by U266 cells renders them susceptible to killing by PR1-CTL and 8F4 antibody in a dosedependent manner (Figure 17). Specifically, NE enhanced the killing of U266 cells by PR1-CTL at 10:1 and 5:1 effector: target (E:T) ratios, when compared to untreated or ova-supplemented U266 cells (Figure 17A). The efficacy of PR1CTL in eliminating PR1-cross-presenting multiple myeloma cells was further 
validated by U266 cells that were supplemented with P3, where killing was demonstrated at the 10:1, 5:1 and 2.5:1 E:T ratios (Figure 17A).

Since we have previously shown that the anti-PR1/HLA-A2 antibody (8F4) Iyses malignant cells via $\mathrm{CDC},{ }^{40,50}$ we tested whether multiple myeloma could be killed by $8 \mathrm{~F} 4$. In a standard CDC assay, we demonstrate significantly higher 8F4mediated killing of U266 target cells that were cultured with NE and P3 (Figure 17B). Combined, these studies show that multiple myeloma is rendered susceptible to killing by PR1-targeting immunotherapies and further confirm PR1 cross-presentation by multiple myeloma. 
Figure 17. PR1 cross-presentation increases the susceptibility of multiple myeloma to PR1-CTL and anti-PR1/HLA-A2 antibody.

A

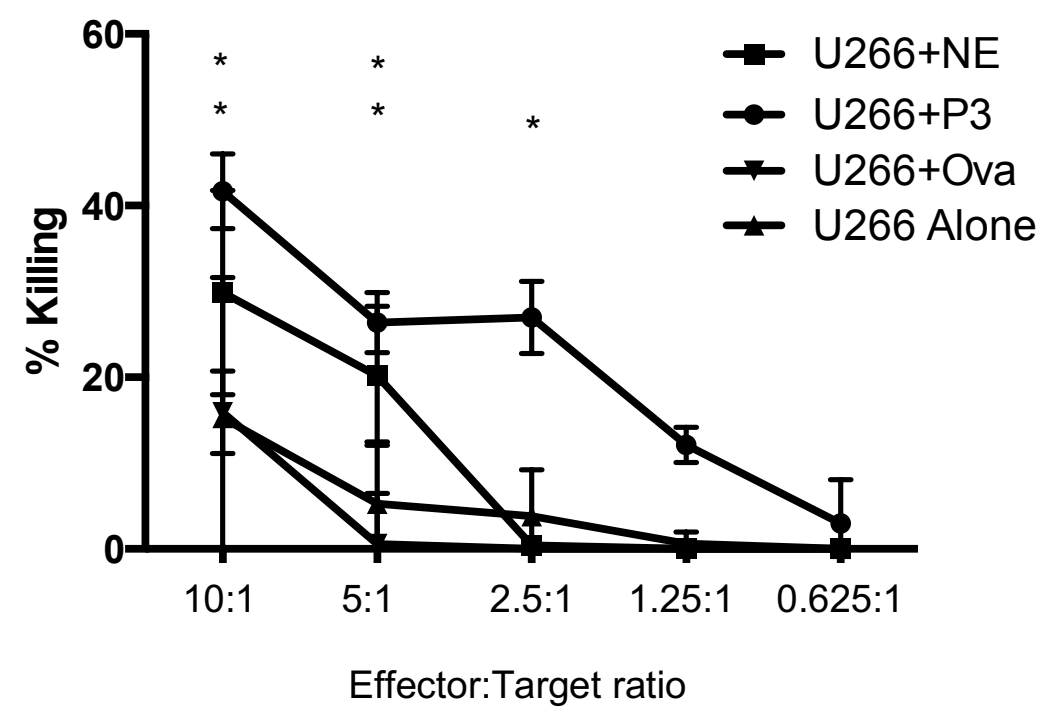

B

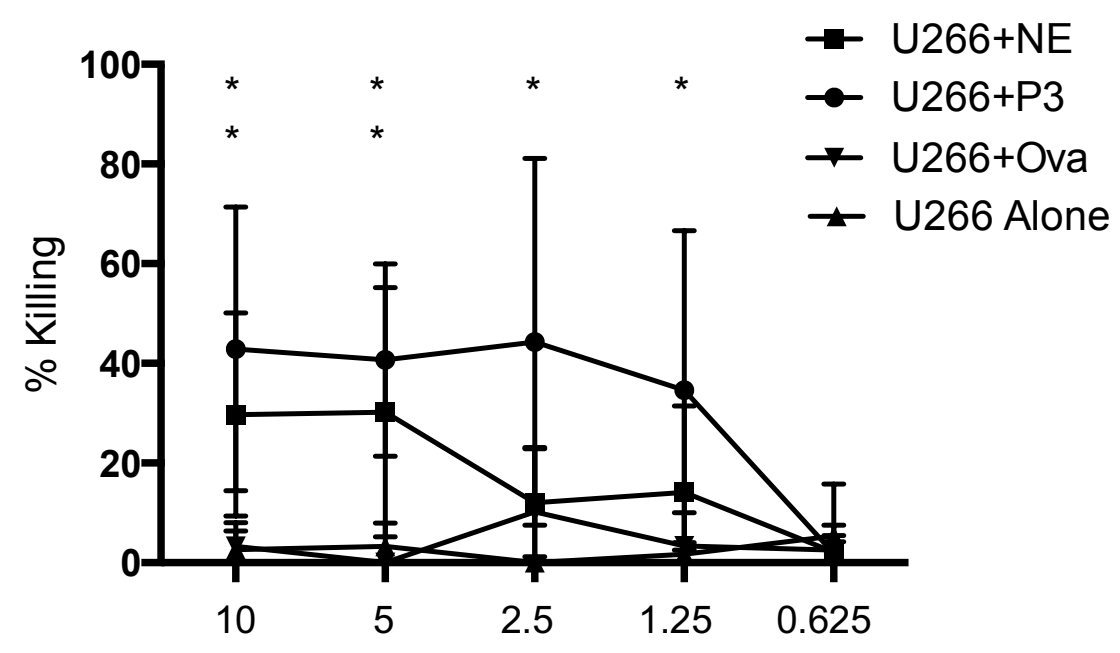

$8 \mathrm{~F} 4$ concentration $(\mu \mathrm{g} / \mathrm{mL})$ 
Figure 17. PR1 cross-presentation increases the susceptibility of multiple myeloma to PR1-CTL and anti-PR1/HLA-A2 antibody. (A) When comparing PR1-CTL killing of target U266 cells supplemented with NE or P3 to ovasupplemented U266 and U266 cells alone, significantly higher killing of target cells was seen at the 10:1 and 5:1 E:T ratios for the U266 + NE group and 10:1, 5:1 and 2.5:1 E:T ratios for the U266 + P3 group $\left({ }^{\star} P<0.05\right)$. (B) Similarly, when comparing 8F4 killing of NE-supplemented and P3-supplemented U266 target cells to ova-supplemented U266 and U266 cells alone, significantly higher killing of target cells was seen at the $10 \mu \mathrm{g} / \mathrm{mL}$ and $5 \mu \mathrm{g} / \mathrm{mL} 8 \mathrm{~F} 4$ concentrations for the NE-supplemented group and at the $10 \mu \mathrm{g} / \mathrm{mL}, 5 \mu \mathrm{g} / \mathrm{mL}$ and $2.5 \mu \mathrm{g} / \mathrm{mL} 8 \mathrm{~F} 4$ concentrations for the P3-supplemented group $\left({ }^{*} P<0.05\right)$. Significantly higher killing was also demonstrated when comparing P3-supplemented U266 to ovasupplemented U266 at the $1.25 \mu \mathrm{g} / \mathrm{mL} 8 \mathrm{~F} 4$ concentration. Statistical analysis was performed using ANOVA test. 


\subsection{PR1-CTL and 8F4 antibody reduce multiple myeloma burden in xenograft mice}

Since PR1 cross-presentation by U266 increases susceptibility to PR1 targeting immunotherapy in vitro, we next investigated whether PR1 can be targeted in vivo using a multiple myeloma U266 xenograft mouse model. ${ }^{54}$ To

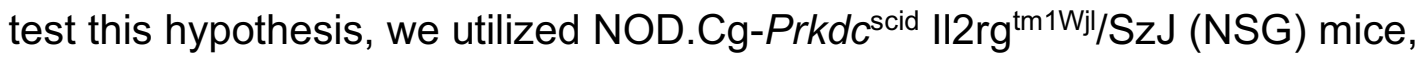
which contain bone marrow resident PMN, providing an available source of $\mathrm{NE}$ and P3 for cross-presentation. Additionally, the murine PR1 sequence is homologous to the human sequence (VLQELNVTV), and murine CTL can recognize PR1/HLA-A2 and are able to be expanded after vaccination. ${ }^{69}$ In this xenograft model, GFP and luciferase transduced U266 cells home to various bone marrow tissues such as the tibia, fibula, spine, and skull of NSG mice within two weeks of engraftment. Multiple myeloma progression can be monitored by IgE ELISA, as U266 is an IgE secreting multiple myeloma subtype, and by bioluminescent imaging (BLI, Figure 18).

After confirming U266 engraftment in the bone marrow and following treatment with $8 \mathrm{~F} 4$, we demonstrate a significantly decreased U266 multiple myeloma burden, as shown by a decrease in the concentration of human IgE in mouse serum in comparison with isotype and untreated groups (Figure 19A). Furthermore, $8 \mathrm{~F} 4$ treatment also noticeably reduced the percent of multiple myeloma cells in mouse bone marrow in comparison to mice treated with isotype and untreated mice (Figure 19B). Similar results were also seen using PR1-CTL 
(Figure 19C). U266 multiple myeloma cells were identified as human CD45 and mouse CD45 cells (Figure 20A and B). Additionally, BLI measurements corroborated these findings (Figure 21). We also observed significant prolonged survival in mice treated with $8 \mathrm{~F} 4$ compared to mice that received isotype antibody (Figure 22). Together, these data suggest that 8F4 and PR1-CTL are a feasible and effective therapy for HLA-A2 ${ }^{+}$multiple myeloma. 
Figure 18. U266 multiple myeloma xenograft model and 8F4 treatment schema.

A

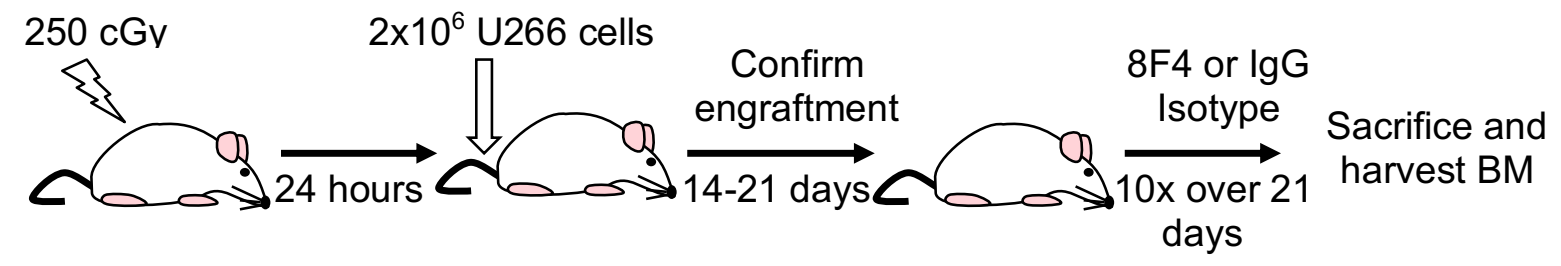

B

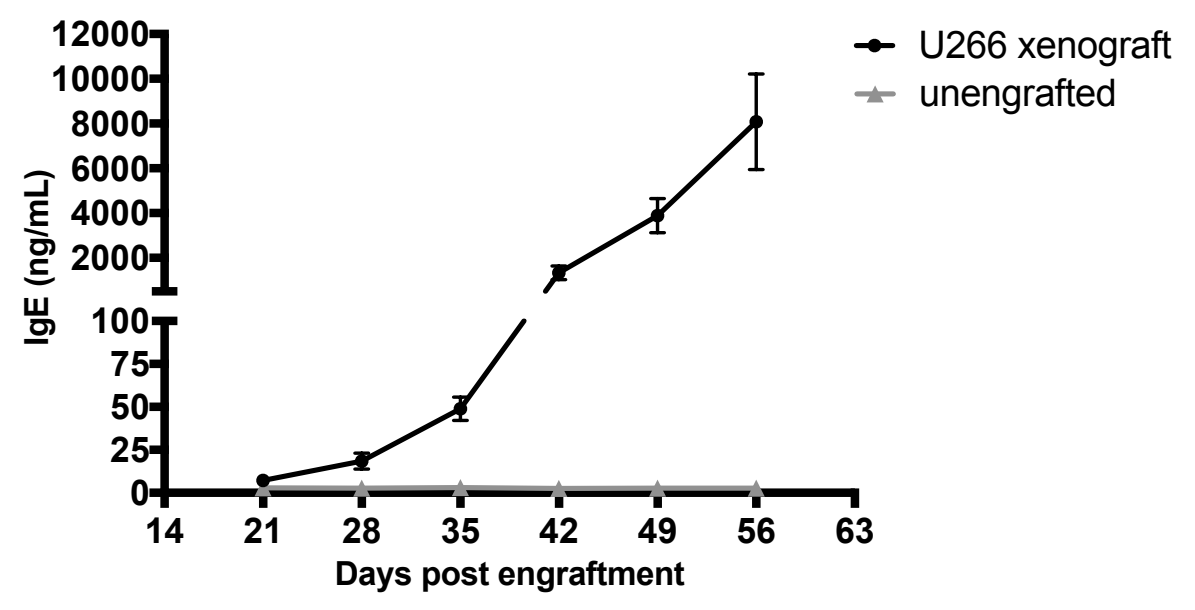

C
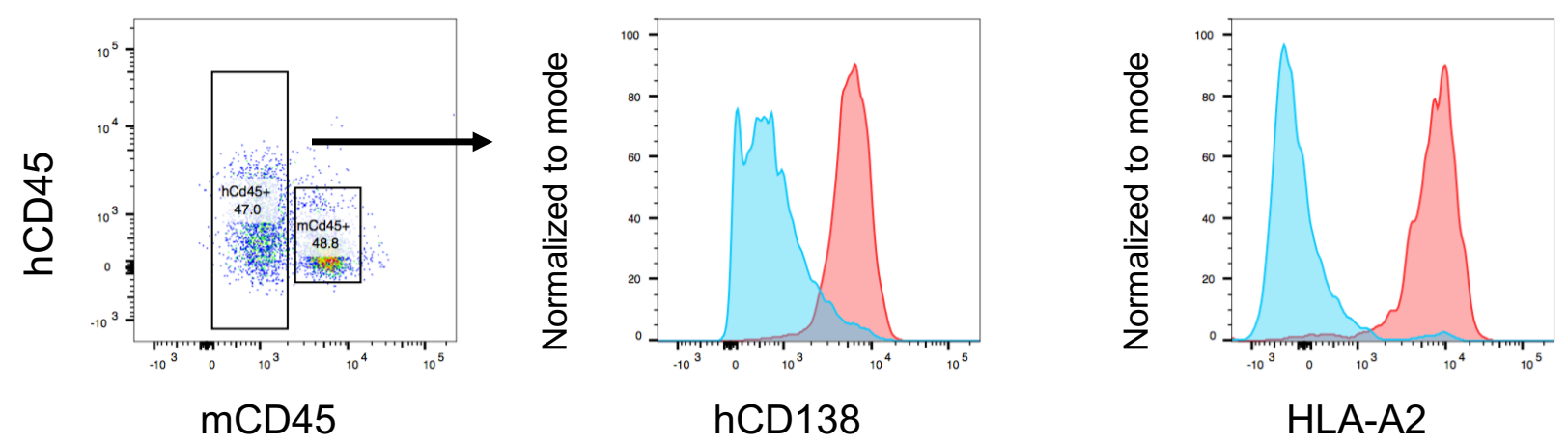
Figure 18. U266 multiple myeloma xenograft model and 8F4 treatment schema. (A) Starting on day 0, female NSG mice were sub-lethally irradiated (250 cGy) prior to U266 engraftment. After 24 hours, on day 1, NSG mice were engrafted with $2 \times 10^{6}$ U266 cells via tail vein injection. Engraftment was monitored and confirmed on days $14-21$ by both IgE ELISA and bioluminescent imaging (BLI, IVIS100). Mice were then received 10 total injections over 21 days of 8F4 antibody or IgG isotype control (Herceptin). Disease progression and treatment effects were monitored by ELISA and BLI. Mice were sacrificed and bone marrow harvested by day 56. (B) Human IgE concentrations in mouse serum were analyzed by ELISA. Mice that were engrafted with U266 cells show increasing accumulation of human IgE over time compared to mice who were unengrafted. (C) U266 cells recovered from mouse bone marrow can be identified from mouse bone marrow by mouse CD45 (mCD45)-negative, human CD45 (hCD45)-positive, human CD138 (hCD138)-positive, or HLA-A2-positive staining. 
Figure 19. 8F4 antibody and PR1-CTL reduce multiple myeloma in U266 xenograft mice.

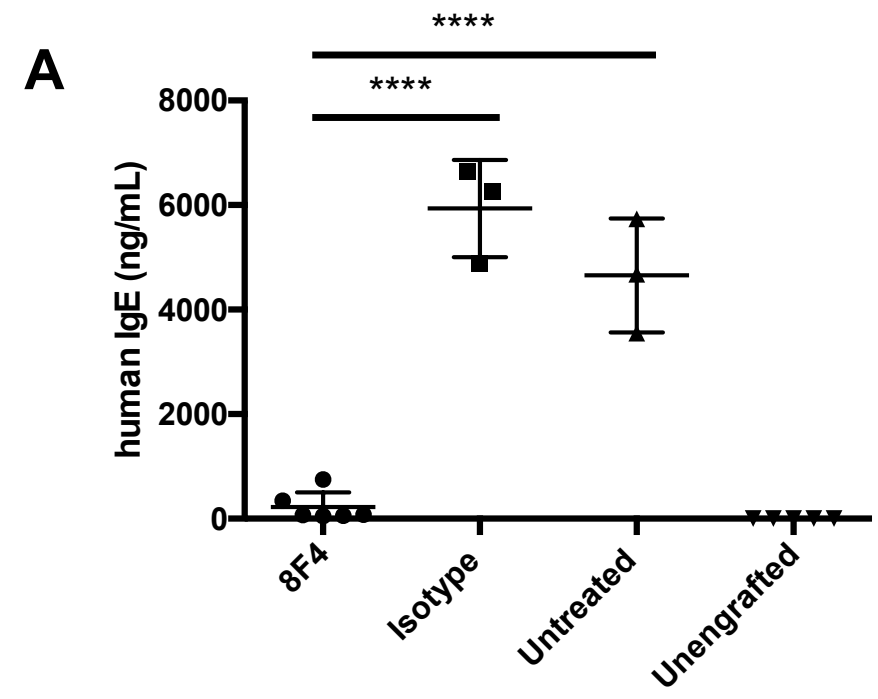

B
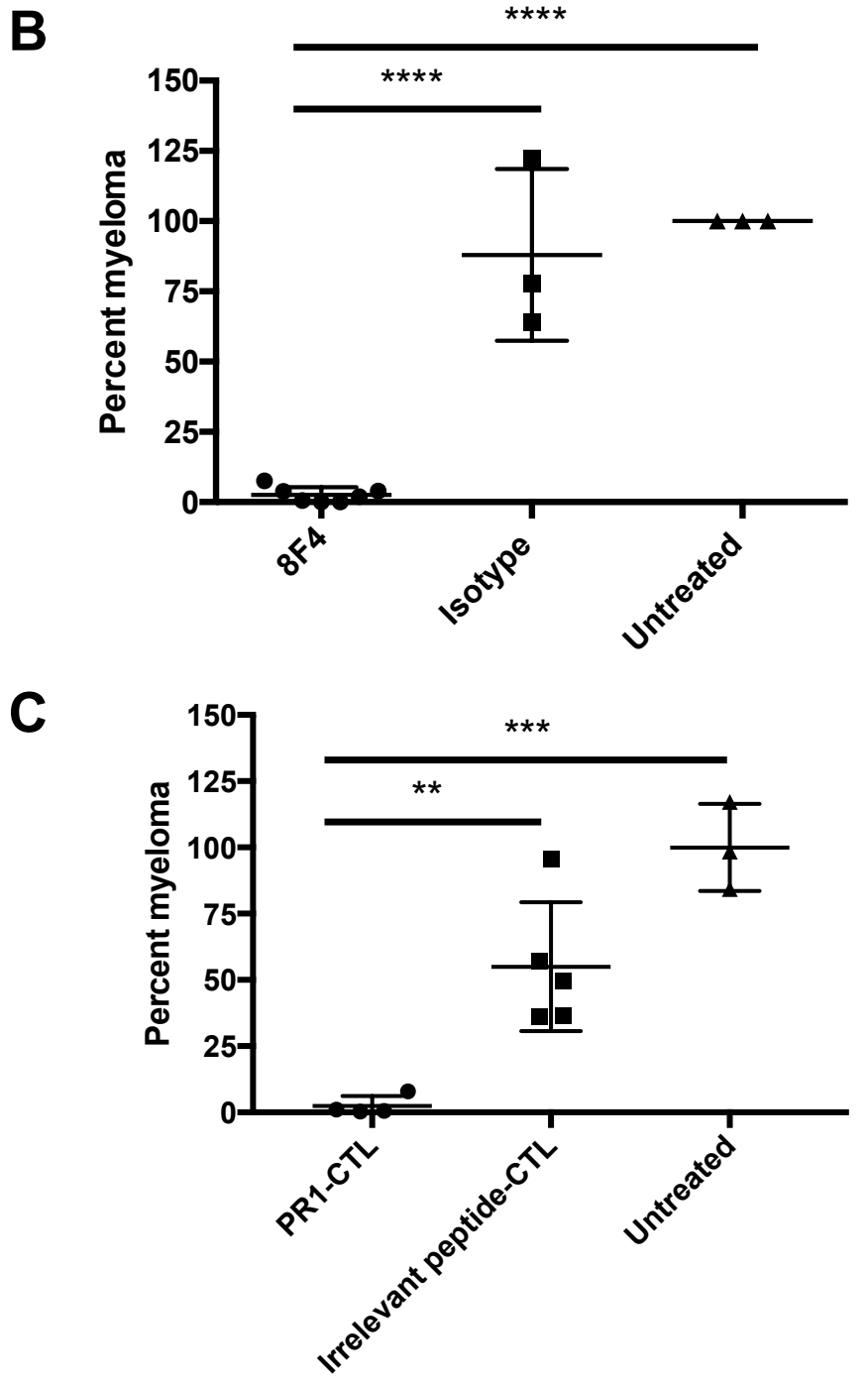
Figure 19. 8F4 antibody and PR1-CTL reduce multiple myeloma in U266 xenograft mice. U266 multiple myeloma xenograft mice were established and treated with (A, B) 8F4 antibody, IgG isotype control, or (C) PR1-CTL. (A) Mouse serum was collected by tail bleeding and separated by centrifugation. Human IgE was measured using ELISA. 8F4 treated mice show reduced circulating human IgE levels compared to isotype and untreated mice $\left({ }^{* \star \star *} P<0.0001\right)$. (B) At the end of treatment mice were sacrificed and bone marrow was harvested, a single cell suspension of bone marrow was generated and analyzed by flow cytometry for residual U266 cells. 8F4 treated mice show reduced percentage of U266 cells in the bone marrow compared to isotype and untreated mice $\left({ }^{* * *} P<0.0001\right)$. Percent of myeloma cells were normalized to untreated mouse bone marrow for each experiment. (C) Mouse bone marrow was analyzed by flow cytometry after treatment with PR1-CTL. PR1-CTL-treated mice show reduced percentage of U266 cells in the bone marrow compared to mice treated with irrelevant peptideCTL $\left({ }^{* *} P<0.01\right)$ or untreated mice $\left({ }^{* * *} \mathrm{P}<0.001\right)$. Statistical analysis was performed using ANOVA test. 
Figure 20. Gating strategy for identifying U266 cells in mouse bone marrow.

\section{A Untreated mouse}

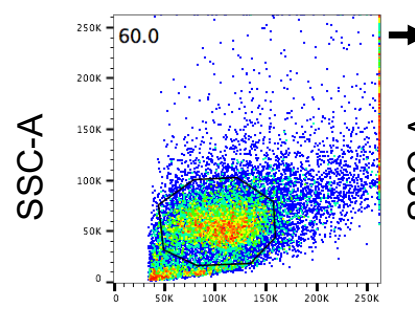

FSC-A

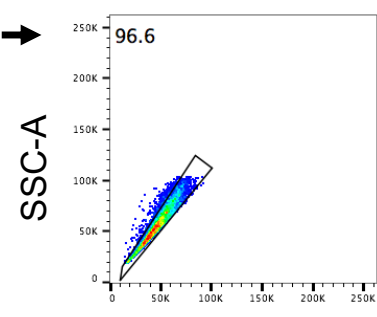

SSC-H

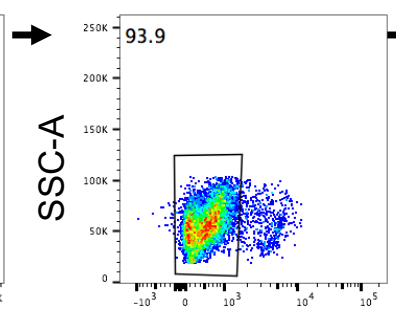

Live/Dead

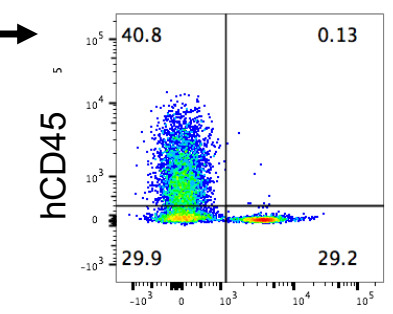

mCD45

\section{B $\quad 8 F 4$ treated mouse}

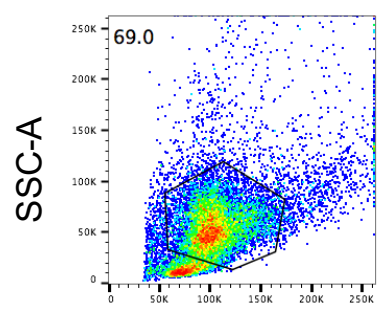

FSC-A

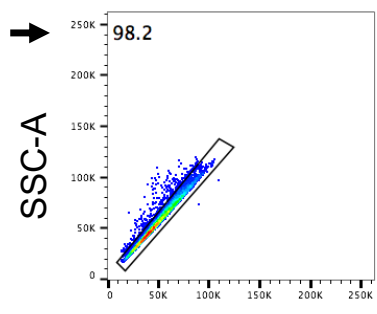

SSC-H

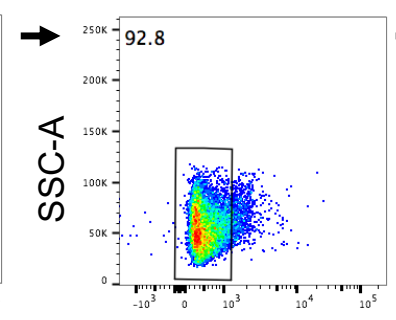

Live/Dead

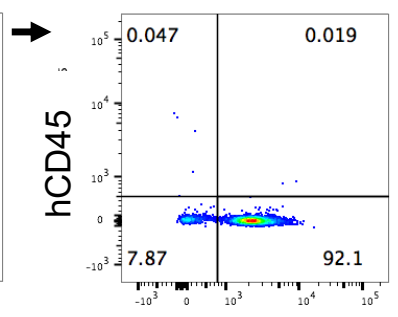

$\mathrm{mCD} 45$

Figure 20. Gating strategy for identifying U266 cells in mouse bone marrow. Gating strategy utilized to identify U266 cells in mouse bone marrow. U266 cells were defined as human $\mathrm{CD} 45^{+}$mouse $\mathrm{CD} 45^{-}$. Bone marrow of a representative mouse that was untreated (A, PBS) shows a robust multiple myeloma burden compared to the bone marrow of an $8 \mathrm{~F} 4$ treated mouse (B). 
Figure 21. 8F4 antibody impedes multiple myeloma growth in U266 xenograft mice.

A
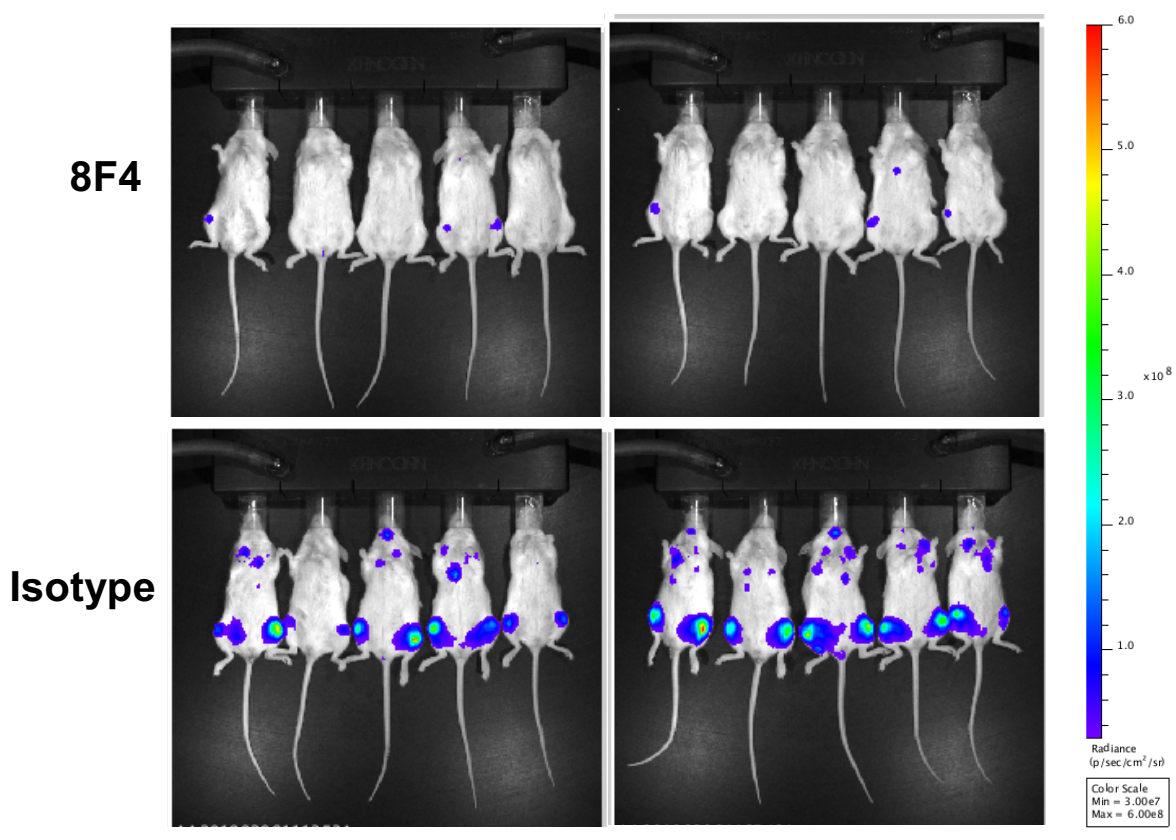

B

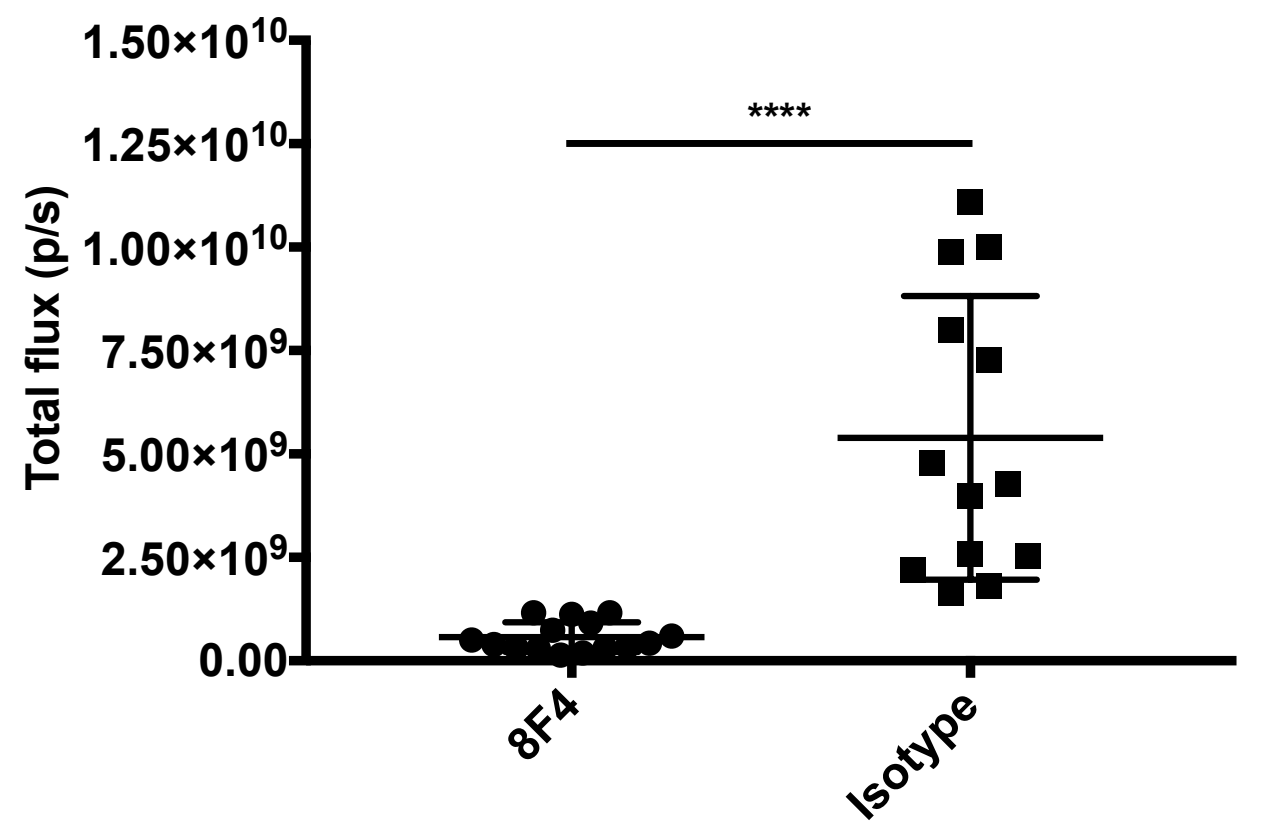


Figure 21. 8F4 antibody impedes multiple myeloma growth in U266 xenograft mice. $8 \mathrm{~F} 4$ antibody treatment reduced multiple myeloma burden and growth. BLI images were captured using an IVIS 100 at the Small Animal Imaging Facility at MD Anderson Cancer center. Bioluminescent images were analyzed using Living Image software (PerkinElmer). All images were set to the same scale before analysis. (A) Representative images of mice treated with either 8F4 antibody or isotype control. (B) BLI images were quantified by measuring total flux $(\mathrm{p} / \mathrm{s})$ from ventral images of mice treated with $8 \mathrm{~F} 4$ antibody or isotype control. $8 \mathrm{~F} 4$ treated mice showed reduced multiple myeloma growth compared to isotype treated mice $\left({ }^{* * * *} P<0.0001\right)$. Statistical analysis was performed using two-tailed T test. 
Figure 22. 8F4 antibody extends survival in U266 xenograft mice

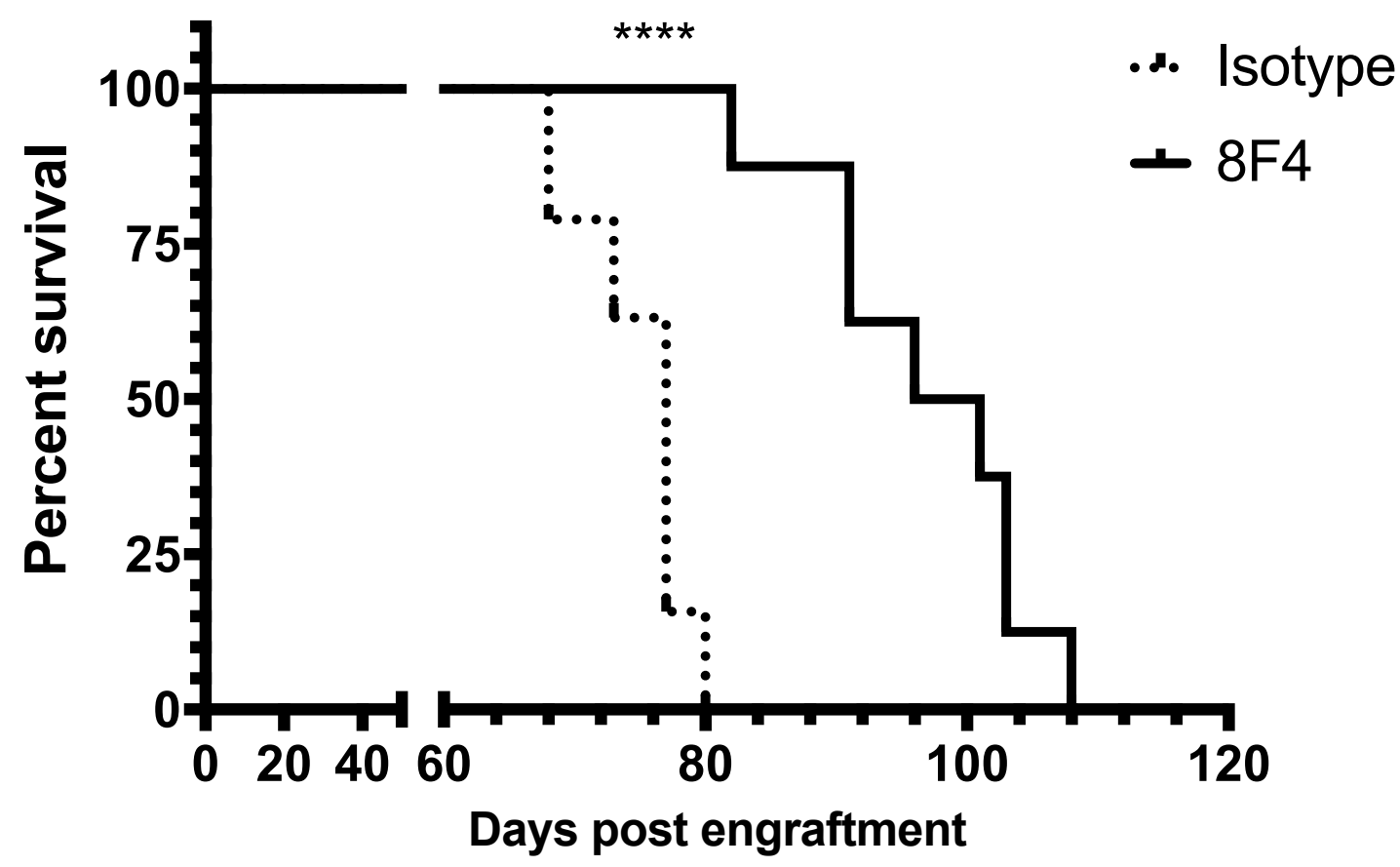

Figure 22. 8F4 antibody extends survival in U266 xenograft mice. U266 xenograft mice were established and treated as previously described in Figure 18 and Figure 19. Time of death for each animal was determined when the mouse became moribund. Kaplan-Meier curves show longer median survival for mice treated with $8 \mathrm{~F} 4$ antibody (median 99 days, $\mathrm{n}=10$ ) compared to mice that received IgG isotype control antibody (Herceptin; median 77 days, $n=20$ ) $\left({ }^{* * *} P<0.0001\right)$. 


\subsection{F4 antibody and bortezomib combination therapy results in an increased anti-myeloma effect}

As combination therapy for multiple myeloma is commonplace already, and new multiple myeloma therapies are frequently being used in combination, we aimed to further our understanding of how $8 \mathrm{~F} 4$ could be integrated into standard of care treatments. To this end, we sought to investigate the interaction between $8 \mathrm{~F} 4$ and bortezomib and identify any potential cooperative effects for treating multiple myeloma. First we observed an additive anti-myeloma effect when 8F4 is combined with bortezomib using an Annexin $\mathrm{V}$ flow-based apoptosis assay in vitro (Figure 23). Greater cell death was noted in U266 cells that were co-cultured with PMN and received combination therapy compared to cells that received either $8 \mathrm{~F} 4$ or bortezomib single agent therapy alone.

Furthermore, we translated our findings using our U266 xenograft model and found that combination therapy of $8 \mathrm{~F} 4$ and bortezomib more effectively reduces the myeloma burden in xenograft mice as well as prolongs survival (Figure 24). As multiple myeloma therapies continue to improve, we also investigated whether improved beneficiary effects could be achieved with nextgeneration proteasome inhibitors, such as carfilzomib. While not significant at this time, mice that received $8 \mathrm{~F} 4$ in combination with carfilzomib appear to have a longer survival rate than those that received bortezomib (Figure 24B). 
Figure 23. 8F4 and bortezomib combination has additive anti-myeloma activity.

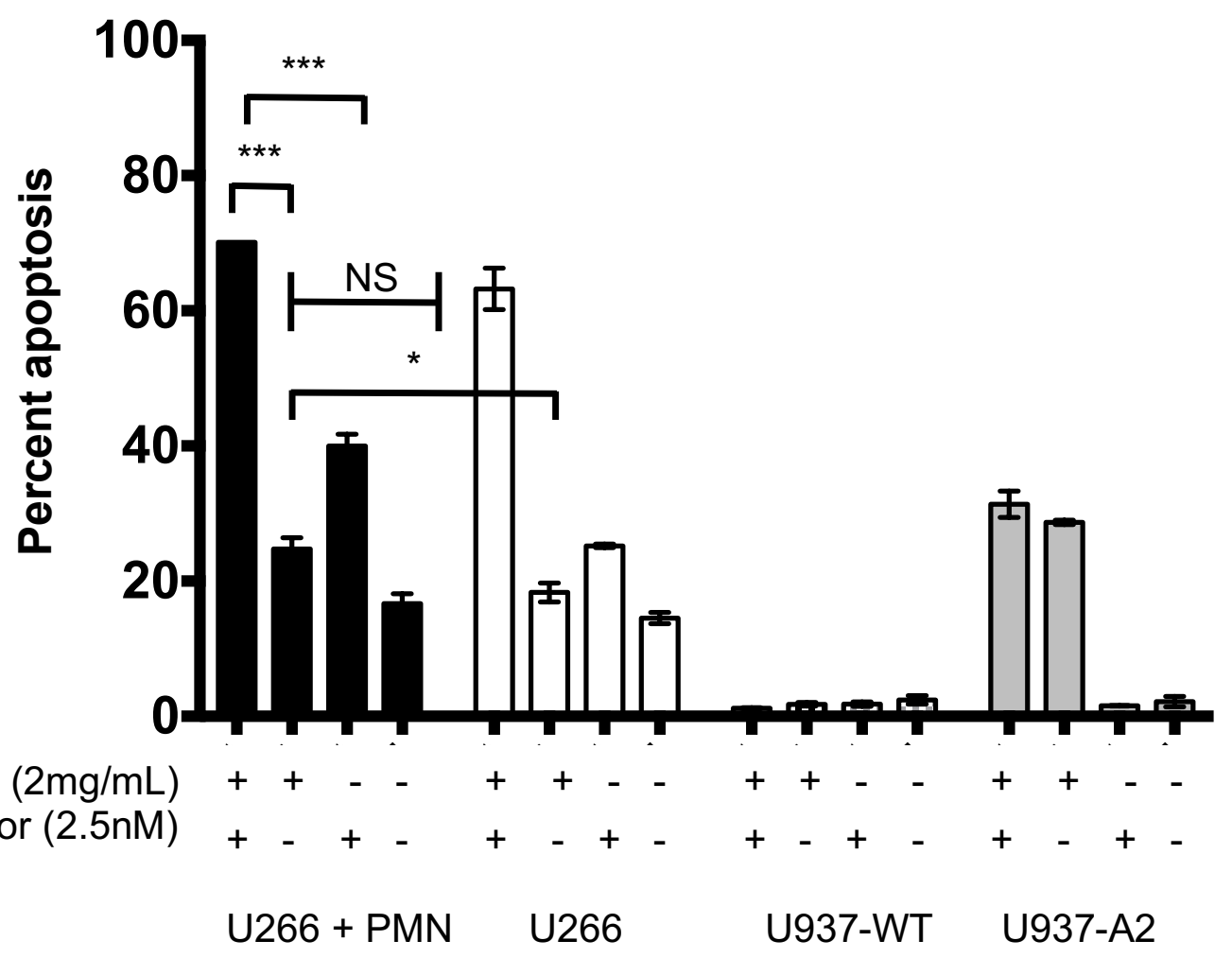


Figure 23. 8F4 and bortezomib combination has an additive anti-myeloma activity. U266 cells were co-cultured with irradiated PMN 24 hours prior to drug exposure to facilitate PR1 cross-presentation (U266 +PMN) or unpulsed as control. U937-WT and U937-A2 AML cell lines were used as negative and positive controls for $8 \mathrm{~F} 4$ mediated lysis respectively. U266 + PMN cells that received $8 \mathrm{~F} 4$ and bortezomib combination therapy showed increased apoptosis via Annexin V and propidium iodide staining compared to U266 + PMN cells that received $8 \mathrm{~F} 4$ alone $\left({ }^{* * *} P<0.001\right)$ or bortezomib alone $\left({ }^{* * *} P<0.001\right)$. Mean \pm SEM of the sum percent of Annexin $\mathrm{V}^{+}$and Annexin $\mathrm{V}^{+}$propidium iodide ${ }^{+}$is shown from triplicate wells. Figure is representative of three independent experiments. Statistical analysis was performed using ANOVA test. NS, not significant. 
Figure 24. 8F4 and bortezomib combination therapy more effectively reduces the multiple myeloma burden in xenograft mice and prolongs survival.

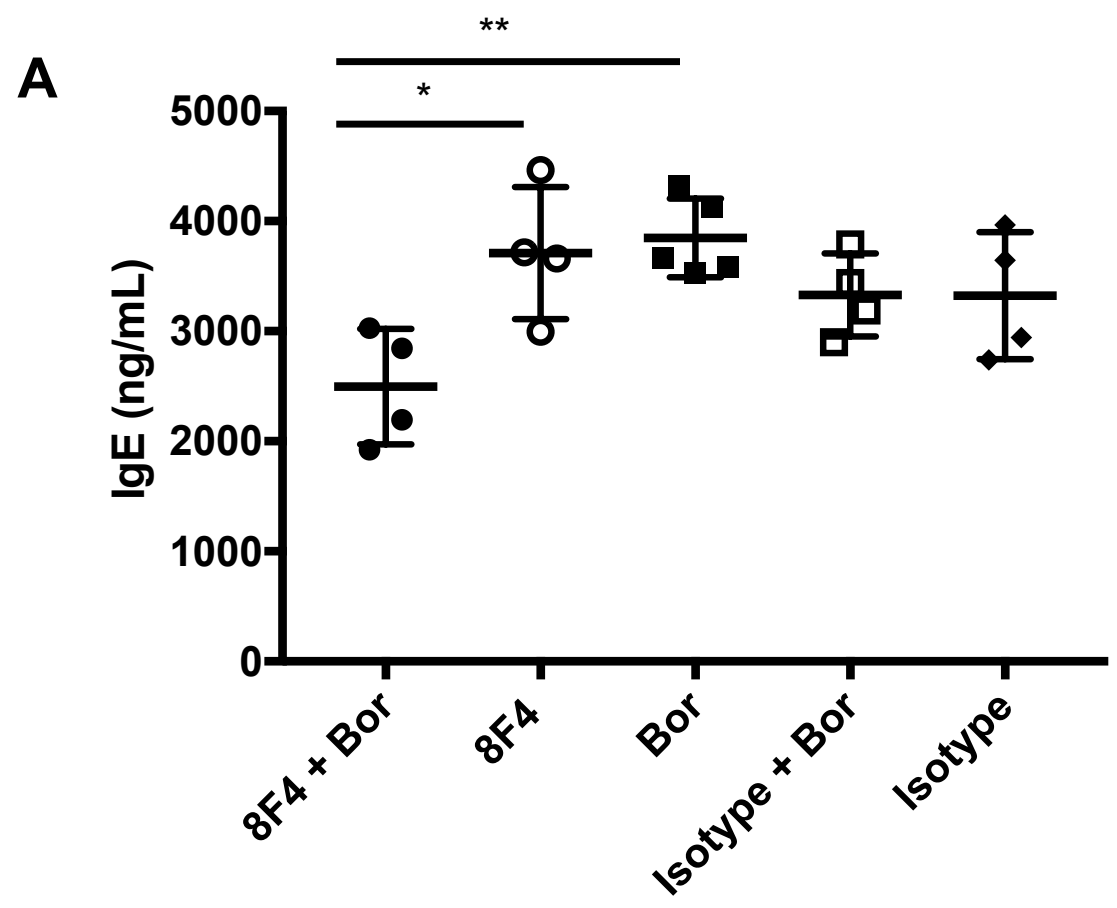

B

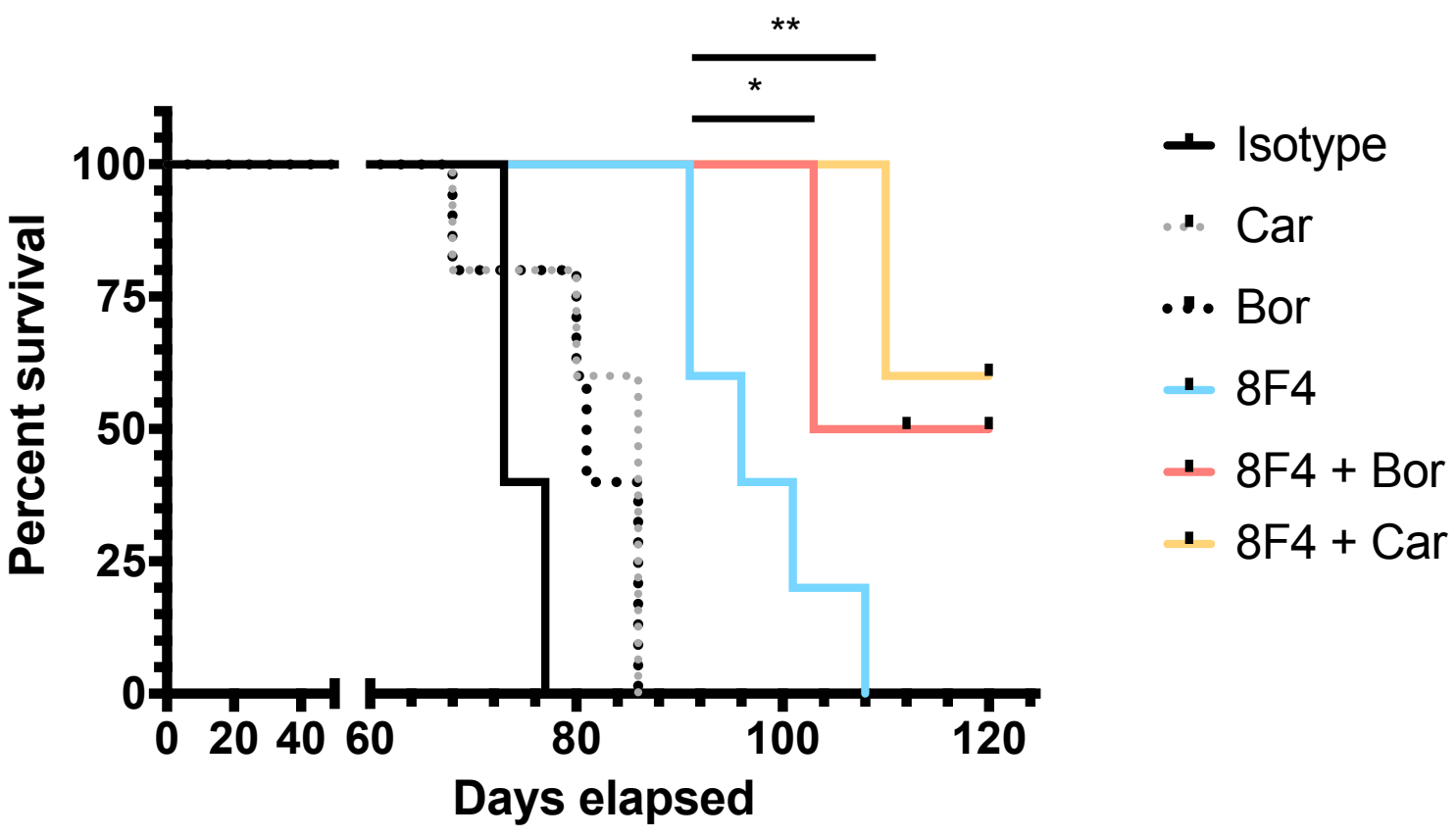


Figure 24. 8F4 and bortezomib combination therapy more effectively reduces the multiple myeloma burden in xenograft mice and prolongs survival. U266 xenograft mice were established as previously described in Figure 18 and Figure 19. (A) Mice were treated with low dose 8F4 antibody (0.05 $\mathrm{mg} / \mathrm{kg}$ ) alone or in combination with bortezomib (Bor, $0.025 \mathrm{mg} / \mathrm{kg}$ ) or isotype control antibody $(1 \mathrm{mg} / \mathrm{kg})$ alone or in combination with bortezomib $(0.025$ $\mathrm{mg} / \mathrm{kg}$ ). Combination therapy of $8 \mathrm{~F} 4$ and bortezomib showed improved reduction in human IgE serum levels in mice compared to those that received single agent treatment of $8 \mathrm{~F} 4\left({ }^{*} P<0.05\right)$ and single agent treatment of bortezomib $\left({ }^{* *} P<0.01\right)$. Statistical analysis was performed using ANOVA test. (B) Prolonged survival was measured up to 120 days in xenograft mice that received combination therapy. Mice were treated with $8 \mathrm{~F} 4$ antibody $(10 \mathrm{mg} / \mathrm{kg})$ alone or in combination with bortezomib $(0.025 \mathrm{mg} / \mathrm{kg})$. Mice were also treated with 8F4 in combination with next generation proteasome inhibitor, carfilzomib (Car, $0.025 \mathrm{mg} / \mathrm{kg}$ ) respectively. Time of death for each animal was determined when the mouse became moribund. Kaplan-Meier curves show longer median survival for mice treated with $8 \mathrm{~F} 4$ antibody in combination with bortezomib (median 111.5 days, $\mathrm{n}=5,{ }^{*} P<0.05$ ) or carfilzomib (median undefined, $n=5,{ }^{* *} P<0.01$ ) compared to mice that received $8 \mathrm{~F} 4$ antibody alone (median 96 days, $\mathrm{n}=5$ ). 


\subsection{PR1/HLA-A2 and PR1-CTL are detected in multiple myeloma patients}

We next investigated whether PR1 could be detected in the bone marrow (BM) from patients with multiple myeloma, and if immunity to PR1 (i.e., PR1-CTL) could be detected in peripheral blood (PB) from patients with multiple myeloma following allo-SCT. We were able to detect PR1/HLA-A2 on the surface of 4 of 8 HLA-A2 ${ }^{+}$patients with multiple myeloma (Table 1; Figure 25). To highlight the low background staining and high specificity for the PR1/HLA-A2 combined epitope of the $8 \mathrm{~F} 4$ antibody, control staining and confocal imaging is was performed on U266 cells pulsed or non pulsed with PMN (Figure 26). 
Table 1. Confocal imaging of PR1/HLA-A2 in multiple myeloma patients.

\begin{tabular}{|c|c|c|c|c|c|c|}
\hline Patient & $\begin{array}{c}\text { MM } \\
\text { subtype }\end{array}$ & $\begin{array}{l}\text { Durie } \\
\text { Salmon } \\
\text { Stage }\end{array}$ & Cytogenetics & $\begin{array}{l}\text { History of } \\
\text { relapse or } \\
\text { progression }\end{array}$ & $\begin{array}{l}\text { Number of } \\
\text { treatments* }\end{array}$ & $\begin{array}{c}\text { PR1/HLA- } \\
\mathrm{A} 2 \\
\text { reactivity }\end{array}$ \\
\hline UPN1 & $\begin{array}{c}\lg \mathrm{A} \\
\text { lambda }\end{array}$ & Stage II & Normal diploid & Yes & 6 & - \\
\hline UPN2 & $\begin{array}{c}\lg G \\
\text { lambda }\end{array}$ & Stage II & Del 13 & Yes & 6 & - \\
\hline UPN3 & $\begin{array}{l}\lg G \\
\text { kappa }\end{array}$ & Stage I & Normal diploid & Yes & 4 & - \\
\hline UPN4 & $\begin{array}{l}\text { lgG } \\
\text { lambda }\end{array}$ & Stage III & Del 17p13 & Yes & 1 & - \\
\hline UPN5 & $\begin{array}{c}\lg A \\
\text { kappa }\end{array}$ & Stage II & Del 13 & Yes & 0 & + \\
\hline UPN6 & $\begin{array}{c}\lg G \\
\text { kappa }\end{array}$ & Stage III & Normal diploid & Yes & 0 & + \\
\hline UPN7 & $\begin{array}{c}\lg G \\
\text { lambda }\end{array}$ & Stage III & Del 13; t(4;14) & Yes & 1 & + \\
\hline UPN8 & $\begin{array}{c}\lg A \\
\text { kappa }\end{array}$ & Stage II & $\begin{array}{c}\text { Del 13; Del } \\
17 p 13 \\
\end{array}$ & Yes & 5 & + \\
\hline
\end{tabular}

*Number of treatments prior to sample acquisition.

Abbreviations: UPN, unique patient number; MM, multiple myeloma; Del, deletion; N/A, not available; $t$, translocation. 
Figure 25. PR1/HLA-A2 is presented on the surface of patient multiple myeloma cells.
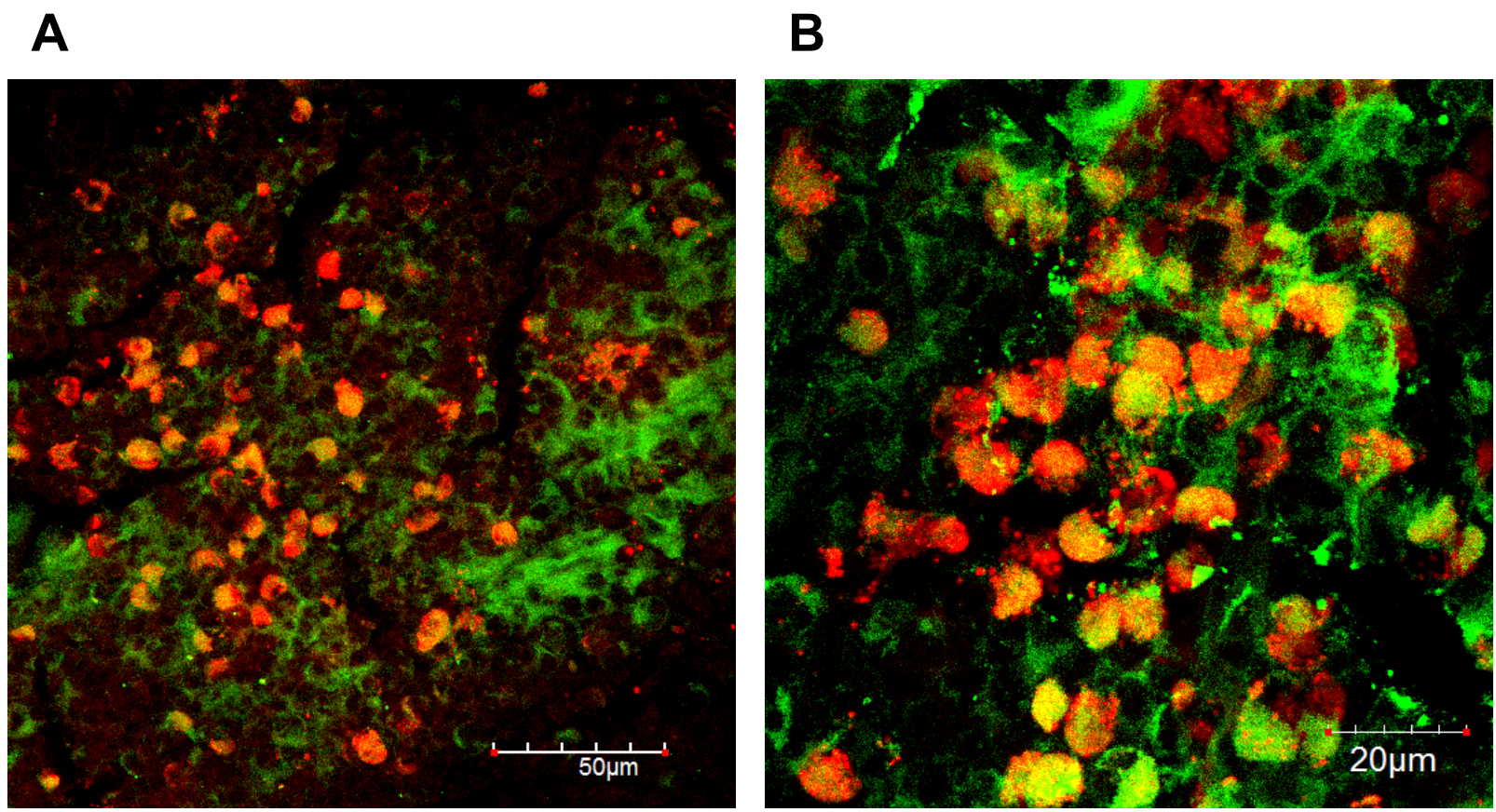

Figure 25. PR1/HLA-A2 is presented on the surface of patient multiple myeloma cells. Bone marrow smears from 2 patients with multiple myeloma were stained with (A) anti-PR1/HLA-A2 (8F4)-647 (red) and anti-HLA-A2-Alex488 (green) or (B) anti-PR1/HLA-A2 (8F4)-647 (red) and anti-CD138-Alex-488 (green) and then imaged using confocal laser microscopy. PR1/HLA-A2 is expressed by multiple myeloma cells as shown by the co-staining of $8 \mathrm{~F} 4$ with HLA-A2 or CD138. 
Figure 26. U266 cross-presents PR1 after exposure to NE/P3 from PMN coculture.

A

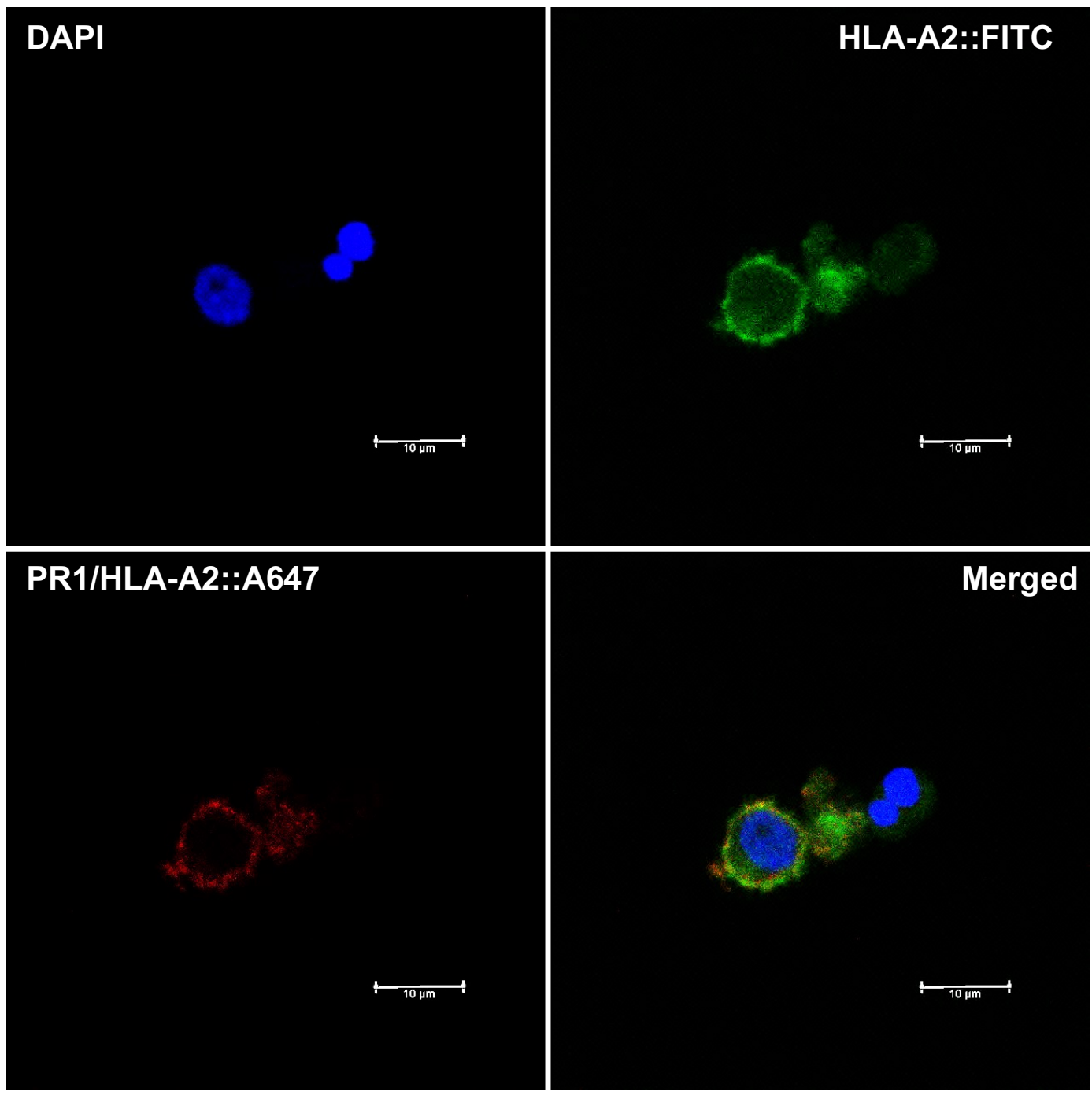


Figure 26. U266 cross-presents PR1 after exposure to NE/P3 from PMN coculture.

B

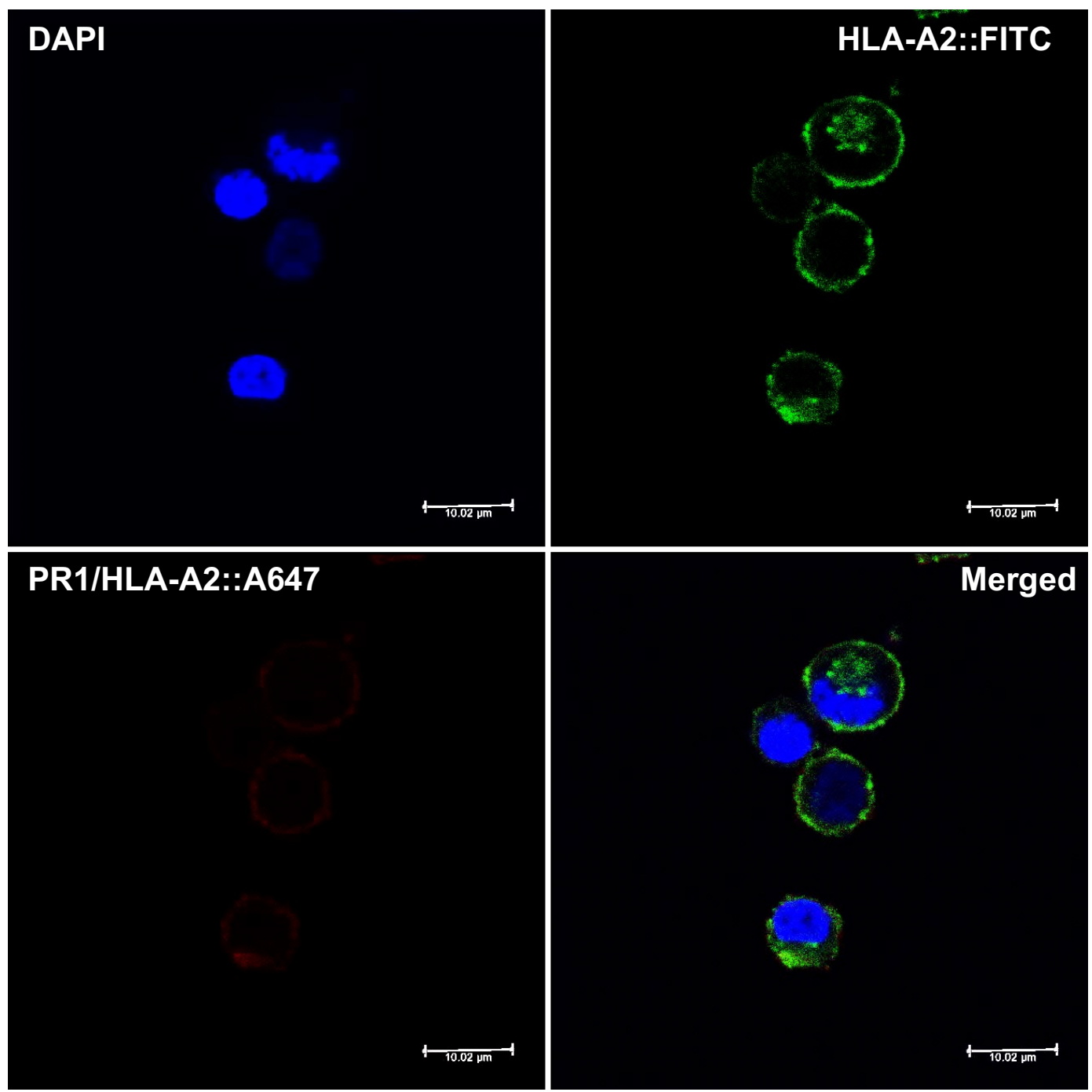

Figure 26. U266 cross-presents PR1 after exposure to NE/P3 from PMN coculture. Control staining of U266 cell line (A) co-cultured with PMN or (B) untreated was evaluated by confocal microscopy. Immunofluorescence staining for Alexa 647-8F4 (red), FITC-HLA-A2 (green) and DAPI stained nuclei (blue) is shown. Data are representative images from three independent experiments. 
To determine whether PR1-CTL could be detected in PB from HLA-A2 ${ }^{+}$ patients with multiple myeloma, we used PR1/HLA-A2 dextramer staining and showed PR1-CTL in the PB from 10 of 14 multiple myeloma patients who received allo-SCT (Table 2; Figure 27 and Figure 28). The median frequency of PR1-CTL in these multiple myeloma patients was $0.053 \%$ of $\mathrm{CD}^{+} \mathrm{T}$ cells (Range, $0 \%-1.67 \%$ ), which is higher than the frequency of PR1-CTL reported in HLA-A2 ${ }^{+}$healthy donors. ${ }^{70}$ 
Table 2. PR1-CTL frequency in HLA-A2 ${ }^{+}$multiple myeloma patients

following allogeneic stem cell transplantation.

\begin{tabular}{c|c|c|c|c}
\hline Patient & MM subtype & $\begin{array}{c}\text { Days post } \\
\text { allo-SCT }\end{array}$ & Alive status & \% dextramer staining \\
\hline UPN4 & IgG lambda & 716 & No & 0.00282 \\
UPN8 & IgA kappa & 378 & No & 0.125 \\
UPN9 & IgG lambda & 490 & No & 0.0192 \\
UPN10 & Unspecified & 1089 & No & 0.002 \\
UPN11 & Lambda light chain & 97 & Yes & 0.255 \\
UPN12 & IgG kappa & 266 & Yes & 0.231 \\
UPN13 & IgG kappa & 197 & Yes & 1.668 \\
UPN14 & IgA lambda & 247 & Yes & 0.088 \\
UPN15 & Kappa light chain & 176 & No & 0.0867 \\
UPN16 & Kappa light chain & 77 & No & 0 \\
UPN17 & IgG kappa & 379 & No & 0 \\
UPN18 & IgG kappa & 93 & No & 0 \\
UPN19 & IgA lambda & 718 & Yes & 0.681 \\
UPN20 & IgA kappa & 163 & No & 0 \\
\hline
\end{tabular}

Abbreviations: UPN, unique patient number; MM, multiple myeloma; allo-SCT, allogeneic stem cell transplantation 
Figure 27. Gating strategy used to determine PR1-CTL frequency in peripheral blood of multiple myeloma patients following allogeneic stem cell transplantation.
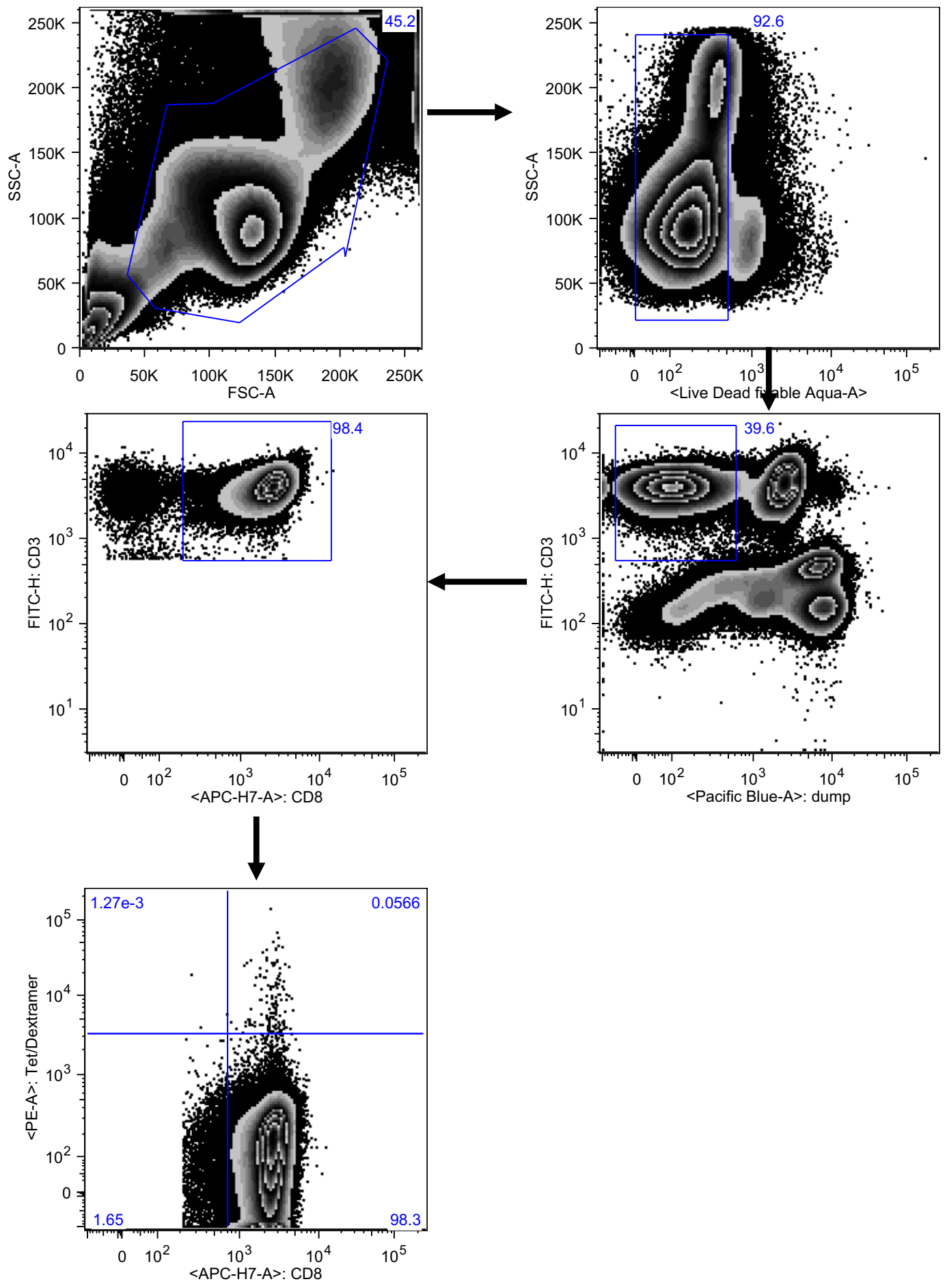
Figure 27. Gating strategy used to determine PR1-CTL frequency in peripheral blood of multiple myeloma patients following allogeneic stem cell transplantation. Cells were stained with CD3, CD8 and PR1/HLA-A2dextramer and the lineage (Lin) markers CD4, CD14, CD16 and CD19. The frequency of PR1/HLA-A2 dextramer ${ }^{+}$cells (i.e. PR1-CTL) was determined as the percent of cells from live, $\mathrm{Lin}^{-}, \mathrm{CD}^{+}, \mathrm{CD} 8^{+}$cells. 
Figure 28. PR1 dextramer plots of multiple myeloma patients following allogeneic stem cell transplantation.

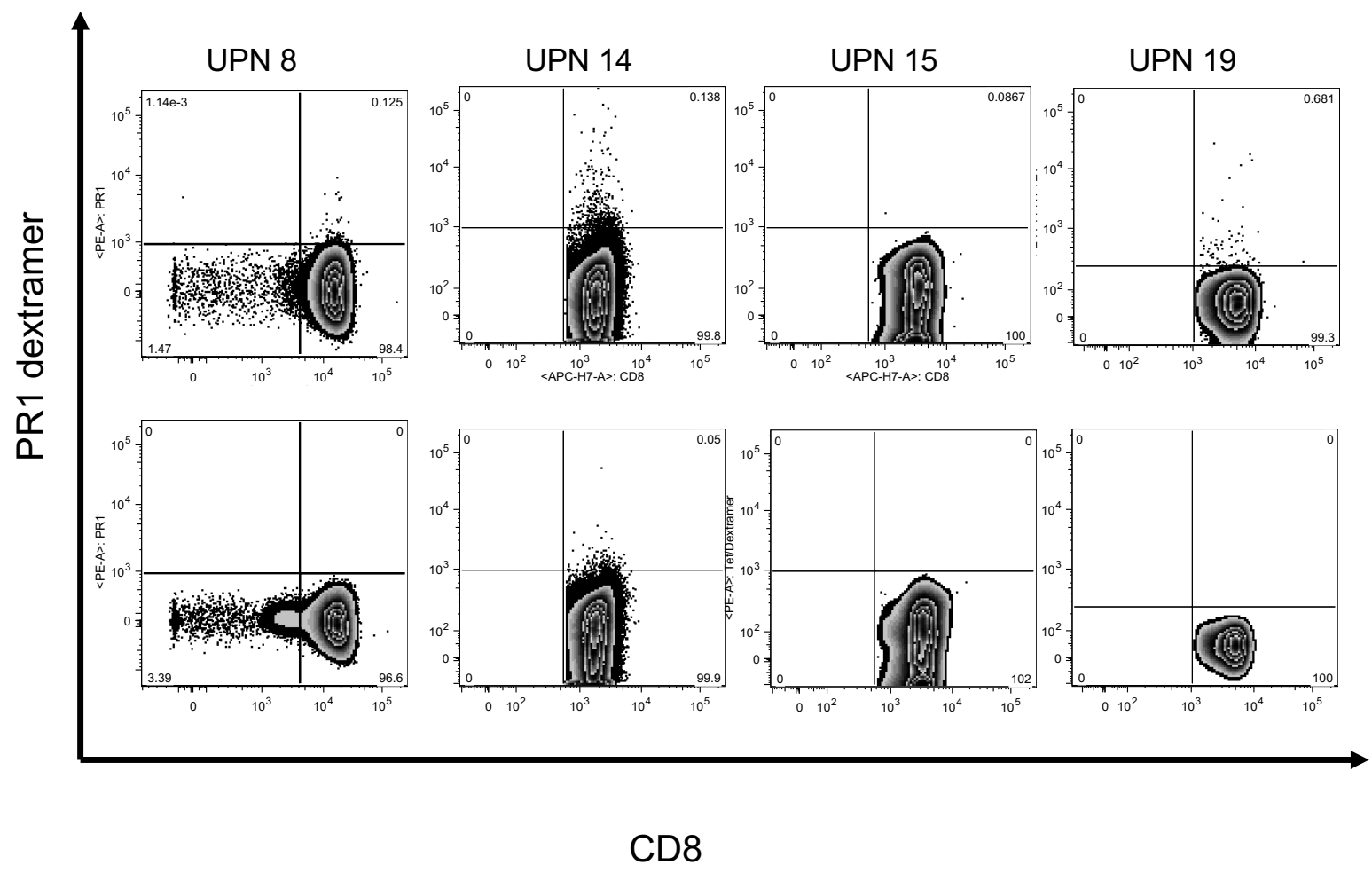

Figure 28. PR1 dextramer plots of multiple myeloma patients following allogeneic stem cell transplantation. Four dextramer plots are representative of the 14 multiple myeloma patients analyzed are shown. Bottom plots represents the FMO staining control for the respective patient plot above. Cells were stained with CD3, CD8 and PR1/HLA-A2-dextramer and the lineage (Lin) markers CD4, CD14, CD16 and CD19. The frequency of PR1/HLA-A2 dextramer ${ }^{+}$cells (i.e. PR1-CTL) was determined as the percent of cells from live, $\mathrm{Lin}^{-}, \mathrm{CD}^{+}, \mathrm{CD} 8^{+}$cells. 
Furthermore, using CD45RA and CCR7 staining, PR1-CTL phenotype was analyzed in 4 of the patients and demonstrated primarily an effector memory phenotype of the PR1-CTL (Figure 29). Additionally, we investigated whether PR1-CTL are present in the PB of HLA-A2 ${ }^{+}$multiple myeloma patients after receiving autologous stem cell transplant (auto-SCT) (Table 3; Figure 30). PR1CTL frequencies were detected by PR1/HLA-A2 tetramer staining in all 18 multiple myeloma patients following auto-SCT. The median frequency of PR1CTL in these patients was $0.205 \%$ (Range, $0.076 \%-1.31 \%$ ). These patient data suggest that bone marrow-derived NE and P3 is taken up and cross-presented by multiple myeloma, and that immunity to PR1 is elicited in multiple myeloma patients following SCT. 
Figure 29. Phenotype of PR1-CTL in patients following allo-SCT.

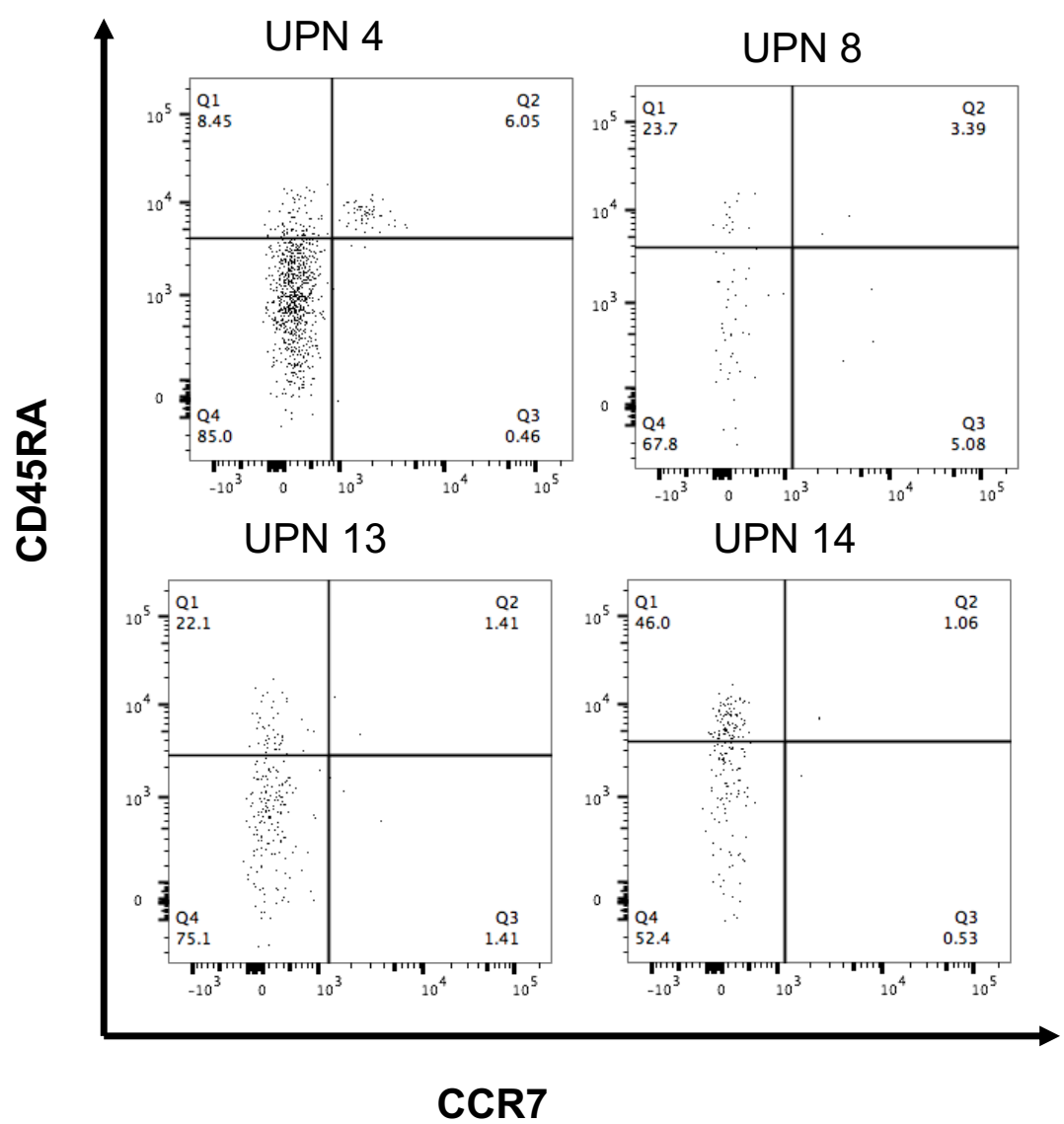

Figure 29. Phenotype of PR1-CTL in patients following allo-SCT. Four multiple myeloma patient samples were stained for PR1-CTL using PR1/HLA-A2 tetramer. Analysis of CCR7 and CD45RA surface expression was performed after gating on the PR1/HLA-A2 dextramer-positive cell population (i.e., PR1CTL). Cells were classified based on the following staining pattern: central memory (CCR7 $\left.{ }^{+} / \mathrm{CD}^{2} 5 \mathrm{RA} \mathrm{A}^{-}\right)$, effector memory (CCR7-/CD45RA'), naïve $\left(\mathrm{CCR}^{+} / \mathrm{CD} 45 \mathrm{RA}{ }^{+}\right)$or terminally differentiated $\left(\mathrm{CCR} 7 / \mathrm{CD}^{2} 5 \mathrm{RA}^{+}\right)$. 
Table 3. PR1-CTL frequency in HLA-A2 ${ }^{+}$multiple myeloma patients

following autologous stem cell transplantation.

\begin{tabular}{c|c|c|c|c}
\hline Patient & MM subtype & $\begin{array}{c}\text { Days post } \\
\text { auto-SCT }\end{array}$ & Alive status & $\begin{array}{c}\% \text { PR1 tetramer } \\
\text { staining }\end{array}$ \\
\hline UPN21 & IgG kappa & 98 & Yes & 0.24 \\
\hline UPN22 & IgG kappa & 154 & Yes & 0.22 \\
\hline UPN23 & IgG kappa & 170 & Yes & 0.29 \\
\hline UPN24 & IgG kappa & 260 & Yes & 0.25 \\
\hline UPN25 & IgG kappa & 1895 & Yes & 0.12 \\
\hline UPN26 & IgG kappa & 32 & Yes & 0.21 \\
\hline UPN27 & IgA kappa & 454 & Yes & 0.081 \\
\hline UPN28 & IgG kappa & 36 & Yes & 0.12 \\
\hline UPN29 & IgG lambda & 962 & Yes & 0.098 \\
\hline UPN30 & Lambda light chain & 104 & Yes & 0.076 \\
\hline UPN31 & Kappa light chain & 392 & Yes & 1.31 \\
\hline UPN32 & IgG lambda & 353 & Yes & 0.28 \\
\hline UPN33 & IgG lambda & 367 & Yes & 0.4 \\
\hline UPN34 & IgG kappa & 301 & Yes & 0.12 \\
\hline UPN35 & IgA kappa & 274 & Yes & 0.13 \\
\hline UPN36 & IgG kappa & 375 & Yes & 0.16 \\
\hline UPN37 & IgG kappa & 351 & Yes & 0.2 \\
\hline UPN38 & IgG kappa & 304 & Yes & 0.48 \\
\hline
\end{tabular}

Abbreviations: UPN, unique patient number; MM, multiple myeloma; auto-SCT, autologous stem cell transplantation 
Figure 30. Gating Strategy used to determine PR1-CTL frequency in peripheral blood of multiple myeloma patients following autologous stem cell transplantation.

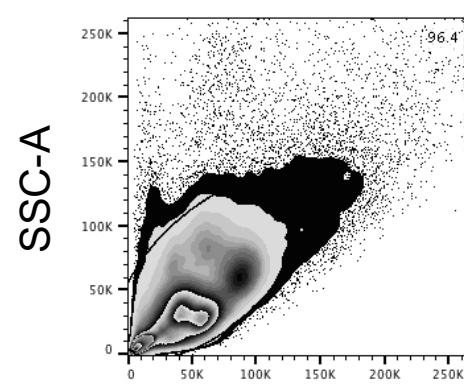

FSC-A

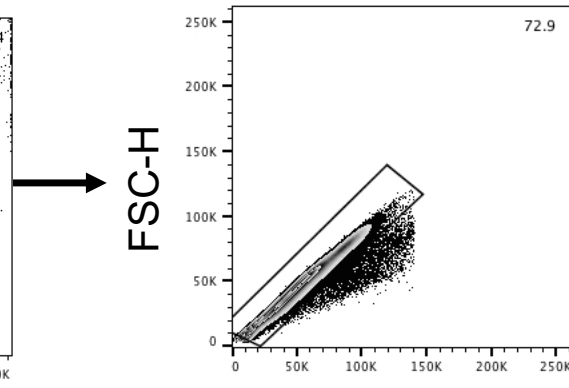

FSC-A
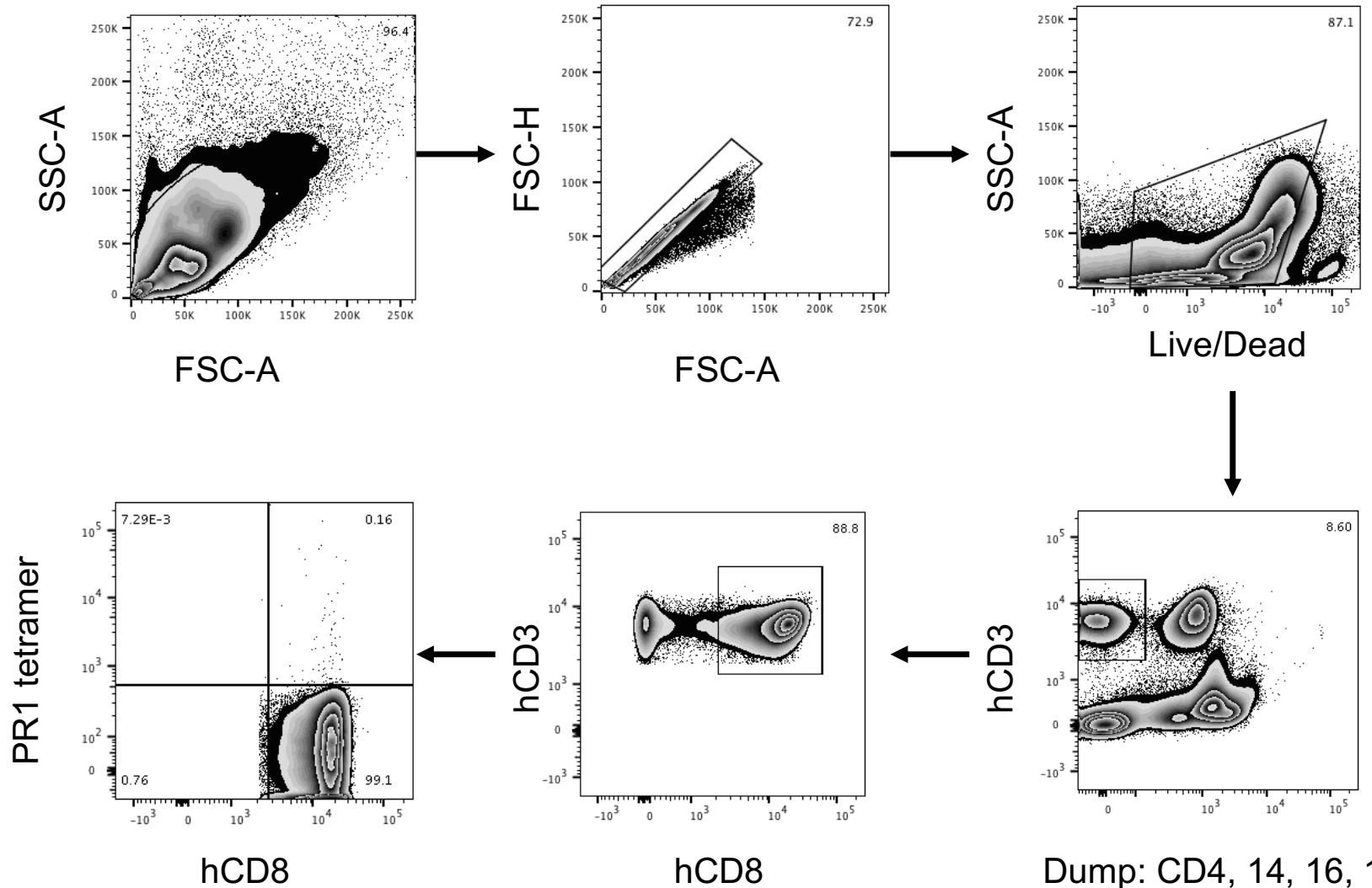

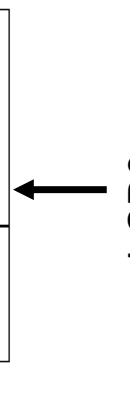

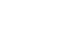
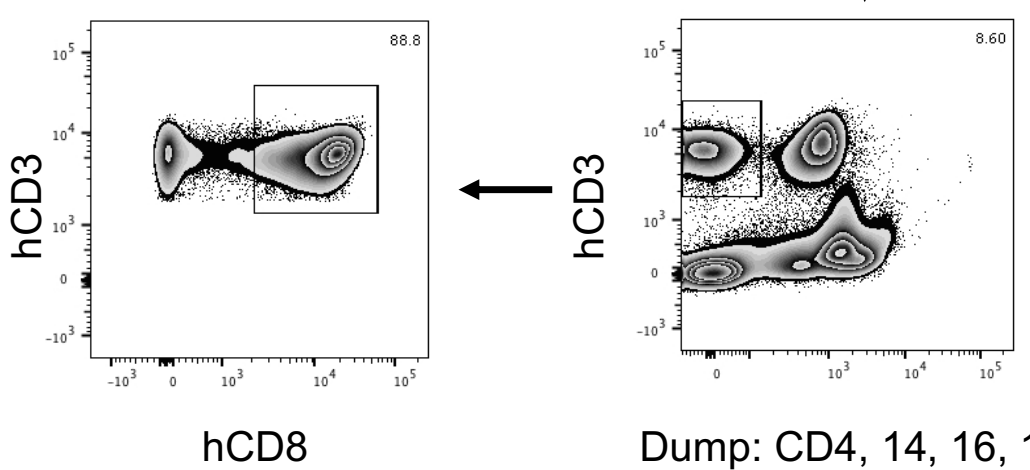

Dump: CD4, 14, 16, 19

Figure 30. Gating Strategy used to determine PR1-CTL frequency in peripheral blood of multiple myeloma patients following autologous stem cell transplantation. Cells were stained with CD3, CD8 and PR1/HLA-A2tetramer and the lineage (Lin) markers CD4, CD14, CD16 and CD19. The frequency of PR1/HLA-A2 tetramer ${ }^{+}$cells (i.e. PR1-CTL) was determined as the percent of cells from live, $\mathrm{Lin}^{-}, \mathrm{CD} 3^{+}, \mathrm{CD} 8^{+}$cells. 
Lastly, we used flow cytometry to study the expression of PR1 on CD138 ${ }^{+}$ multiple myeloma cells from patient bone marrow (to corroborate confocal microscopy data [Table 1; Figure 25]) and to also investigate the expression of co-stimulatory molecules on PR1/CD138 ${ }^{+}$multiple myeloma cells. Our data show surface expression of PR1 and co-stimulatory molecules HLA-DR, CD40, CD80 and CD86 on CD138 multiple myeloma cells in 12 out of 12 total patients (Table 4; Figure 31), further supporting the observation that patient multiple myeloma cells are capable of PR1 cross-presentation, and possibly crosspriming. 
Table 4. Co-stimulatory molecule expression of PR1/HLA-A2+ multiple myeloma patients.

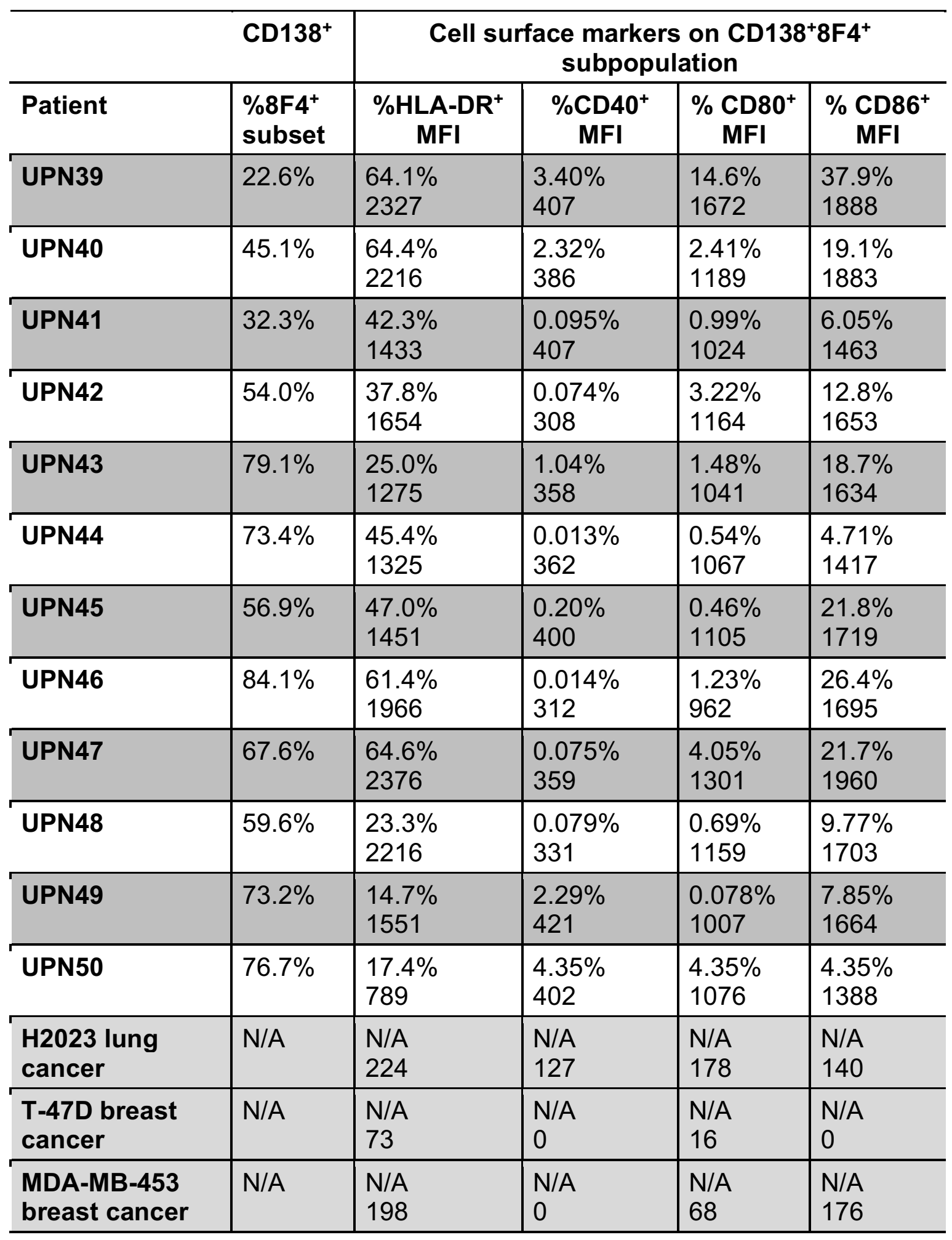


Table 4. Co-stimulatory molecule expression of PR1/HLA-A2 ${ }^{+}$multiple myeloma patients.

Abbreviations: UPN, unique patient number; MFI, median fluorescence intensity;

N/A, not applicable. H2023 lung cancer, T-47D breast cancer, MDA-BM-453

breast cancer cell lines used as negative controls. 
Figure 31. Gating Strategy used to determine PR1 expression and costimulatory molecule expression on patient bone marrow multiple myeloma cells.
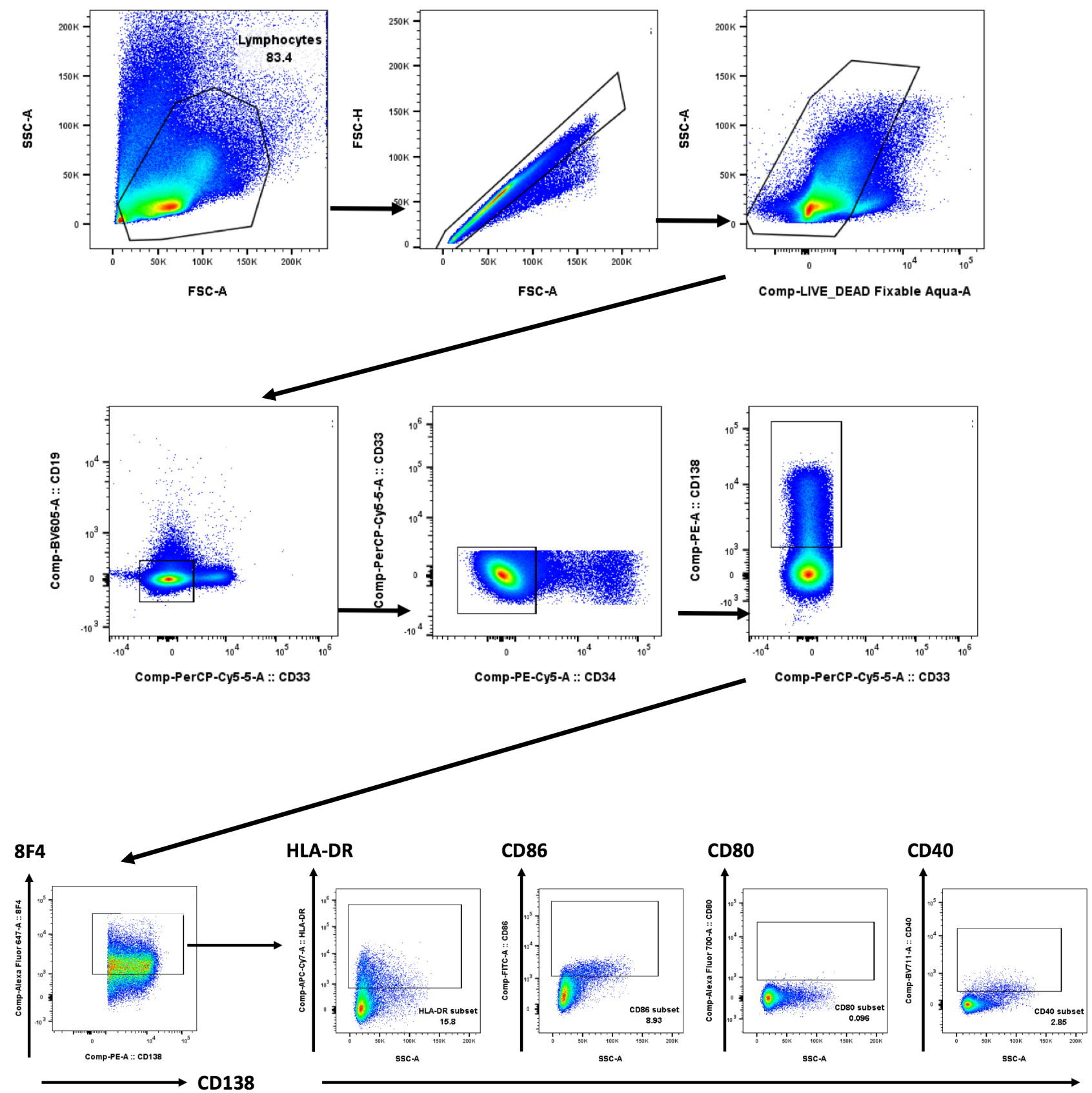
Figure 31. Gating Strategy used to determine PR1 expression and costimulatory molecule expression on patient bone marrow multiple myeloma cells. Cells were stained with CD19, CD33, CD34, CD38, CD138, and HLAA2/PR1 antibody (8F4) to identify PR1 expressing multiple myeloma cells. Analysis of surface expression for CD40, CD80, CD86, and HLA-DR was performed after gating on the PR1/HLA-A2 and CD138 double positive cell population. 


\subsection{Chapter 5 Summary}

This work illustrates that cross-presented antigens can be effectively targeted using immunotherapy in the setting of multiple myeloma. We have demonstrated that multiple myeloma can cross-present PR1, and consequently be targeted using PR1-targeting immunotherapy using PR1-CTL and the 8F4 antibody in vitro and in vivo. Utilizing in vitro cytotoxicity assays, U266 were eliminated after being cultured in media supplemented with soluble NE or P3 by PR1-CTL and 8F4 antibody. Additionally, we utilized a multiple myeloma xenograft mouse model, which illustrated that PR1-CTL and 8F4 antibody treatment can drastically reduce the multiple myeloma burden in mice and extend animal survival.

Additionally, we observed the presence of PR1-CTL in the peripheral blood and bone marrow of multiple myeloma patients. PR1-CTL were detected in the peripheral blood of patients after allo- and auto-SCT and were primarily observed to be effector memory cells. Collectively, this suggests that there is the potential to elicit immunity against PR1 in multiple myeloma patients. Our data also indicate that PR1-expressing multiple myeloma cells from patients express the co-stimulatory molecules HLA-DR, CD40, CD80, and CD86. This finding suggests that multiple myeloma cells could be capable of cross-priming, a potential ancestral role from their B cell origin. 
Overall our work has illustrated that cross-presentation of PR1 renders multiple myeloma susceptible to PR1-targeting immunotherapies. 


\section{Chapter 6: Discussion}

In summary, our work demonstrates the novel ability for multiple myeloma cells to take up and cross-present NE and P3. Multiple myeloma cells lack endogenous NE and P3 expression but possess the ability to internalize these proteins from soluble or cell-associated sources. This internalization leads to the processing of NE and P3 by the proteasome and the subsequent loading of PR1 peptide onto HLA-A2 molecules in a TAP-dependent manner. This process ultimately results in PR1/HLA-A2 expression by multiple myeloma through crosspresentation. Consequently, PR1 cross-presentation leads to the ability to target and eliminate multiple myeloma cells by PR1-targeting immunotherapies such as PR1-CTL or 8F4 antibody (Figure 32). Overall, our work has identified crosspresentation as a novel mechanism by which multiple myeloma expresses tumor antigens and underlines the therapeutic potential of PR1-targeting immunotherapies for the treatment of multiple myeloma. This suggests that the antigen landscape in myeloma is broader than previously believed. This newly appreciated and expanded antigen repertoire may lead to the discovery of a new class of multiple myeloma antigens and the development of new immunotherapies.

The antigenic library of multiple myeloma is rapidly expanding. As briefly mentioned in the introduction of this dissertation, there have been several recent and promising antigens being investigated for the targeting multiple myeloma (CD38, SLAMF7, BCMA). New approaches using immunoprecipitation and mass 
spectrometry are further expanding the potential antigenic profile of multiple myeloma. ${ }^{71}$ However, these endeavors have focused primarily on endogenous peptides and to date have not considered cross-presented peptides as potential immunotherapeutic targets. Our work further expands the potential antigenic repertoire of multiple myeloma, illustrated by the ability to target a crosspresented myeloid leukemia antigen, PR1.

Cross-presentation is an important link between the innate and adaptive immune response. Defects in cross-presentation results in the inability to mount a proper $\mathrm{CD} 8^{+} \mathrm{T}$ cell response against exogenous antigens from viral or tumor sources. ${ }^{72,73}$ Classically, B cells are considered to have cross-presentation capabilities; being able to cross-present antigens and prime CD8 ${ }^{+} \mathrm{T}$ cells. ${ }^{74,75}$ Additionally, plasma cells have also been implicated in priming T cells via crosspresentation. ${ }^{76}$ Our work here is the first to reveal that multiple myeloma is capable of antigen cross-presentation. Interestingly, studies have shown CD38 ${ }^{+}$ plasma cells in multiple myeloma patients expressed a range of co-stimulatory molecules (CD40, CD80, CD86, HLA-DR), although at low levels. ${ }^{76,77}$ In one specific study, Pérez-Andrés and colleagues observed a progressive decrease in the co-stimulatory capacity in malignant plasma cells from MGUS to multiple myeloma. ${ }^{77}$ These observations are corroborated by our own data, which show a small subset of multiple myeloma cells express these co-stimulatory molecules on the cell surface. Together, these data suggest that there may exist 
immunological scenarios where multiple myeloma could function as an APC and prime a $\mathrm{CD} 8^{+} \mathrm{T}$ cell response.

The proteasome plays a prominent role in both antigen presentation and cross-presentation. Our work highlights the importance of the proteasome in PR1 cross-presentation by multiple myeloma. When multiple myeloma cells were treated with the proteasome inhibitors, lactacystin or bortezomib, a significant reduction in cross-presentation of $\mathrm{NE}$ and $\mathrm{P} 3$ was observed, noted by the decrease in PR1/HLA-A2 surface expression. The role of the proteasome in cross-presentation by multiple myeloma is particularly important in the context of bortezomib; a front line therapy administered to newly diagnosed myeloma patients.

Currently, the precise effects of bortezomib and proteasome inhibition on multiple myeloma have yet to be fully elucidated. In our investigations, treating multiple myeloma cells with bortezomib in vitro resulted in decreased PR1 crosspresentation and global HLA-A2 surface expression. However, others have shown that following bortezomib treatment, an increase in antigen presentation was observed. ${ }^{78,79}$ Proteins can be processed by various mechanisms, specifically through cytoplasmic or lysosomal peptidases, bypassing the proteasome. Potentially NE and P3 could also be processed by these peptidases or loaded through the alternative vacuolar pathway, leading to PR1 crosspresentation even in the context of proteasome inhibition. Our own findings have 
also challenged the idea that proteasome inhibition is damaging to antigen presentation. Our in vivo studies, where combination 8F4 antibody and bortezomib were administered to U266 xenograft mice resulted in a more potent anti-myeloma effect by extending animal survival in the U266 xenograft model. Furthermore, when we investigated this cooperative anti-myeloma effect in vitro using flow cytometry based apoptosis assays, we also observed increased cell death when both drugs were used in combination. Since this contradicts our data showing that proteasome inhibition decrease PR1/HLA-A2 expression on the cell surface in vitro, we hypothesize that bortezomib could be suppressing global antigen presentation in multiple myeloma, potentially reducing the background peptide noise on the myeloma cell surface. This could allow for an enrichment of PR1/HLA-A2 expression for targeting by 8F4 antibody. Also, the enhanced killing of multiple myeloma by the combination of $8 \mathrm{~F} 4$ and bortezomib may be due to mechanisms unique to $8 \mathrm{~F} 4$, whereby proteasome inhibition could potentiate the direct mechanism of action of $8 \mathrm{~F} 4$; this work is currently being investigated by our group.

The proteasome is inherently complex. Studies have even reported that peptide generation via proteasome degradation to be mostly a stochastic process. In vitro experiments on the SIINFEKL peptide, derived from ovalbumin, demonstrated that the proteasome cleaves through this peptide $90 \%$ of the time.$^{80}$ This indicates that the proteasome is generally more destructive to peptides than productive. Therefore it could be possible that the low 
concentration of bortezomib may reduce the probability of PR1 peptide destruction in the cells that end up presenting PR1. However, these hypotheses would need to be further validated through experimentation. It is clear that a greater understanding of the effects of proteasome inhibition on cross-

presentation are needed. Specifically, further studies into the mechanistic activity of both $8 \mathrm{~F} 4$ antibody and bortezomib are necessary and warranted, especially in the area of multiple myeloma where combination therapy is commonplace.

The other class of anti-myeloma drugs, IMiDs, does not appear to have any adverse effects on antigen presentation. Our work demonstrated that lenalidomide did not affect the expression of HLA-A2 nor PR1/HLA-A2 expression on the surface of multiple myeloma. While more consideration is needed when potentially combining PR1-targeting immunotherapy with standard of care therapies such as bortezomib, PR1 immunotherapies may be more readily integrated and combined with IMiDs.

The ER appears to be the major site for PR1 loading onto HLA-A2 in multiple myeloma. When treated with an ER to Golgi transport inhibitor, brefeldin $A$, we observed a significant decrease in PR1/HLA-A2 surface expression on multiple myeloma cells. This suggests that PR1 cross-presentation follows the cytosolic pathway model for cross-presentation. Our work has also demonstrated that TAP plays a prominent role in PR1 cross-presentation, as silencing TAP resulted in decreased recognition of PR1/HLA-A2 by PR1-CTLs. These 
observations further support that PR1 cross-presentation follows the cytosolic pathway model. However, we have not conducted the experiments to definitively rule out the roles of the vacuolar or peptide-exchange models in PR1 crosspresentation. It is possible that PR1 can be cross-presented via all three mechanisms at some level of efficiency-a consideration that could aid in explaining the contradictory effects observed with bortezomib proteasome inhibition. Others have reported mechanisms in DCs where the HLA-A2 restricted peptide, gp100, is efficiently cross-presented via the vacuolar pathway in a TAPindependent manner. ${ }^{81}$ Interestingly, it was further demonstrated that the vacuolar pathway for cross-presentation is not exclusively TAP-independent. ${ }^{82}$ While our data illustrate that the cytosolic pathway and the ER and TAP play a prominent role in the cross-presentation of PR1 by multiple myeloma, it is becoming clear that the models of cross-presentation are not mutually exclusive. Cells may utilize a combination or multiple pathways for effective antigen crosspresentation.

Interestingly, our PR1 tetramer findings demonstrate that the TCR repertoire of multiple myeloma patients contains TCRs that recognize PR1 after allo- and auto-SCT. These data further support the feasibility of targeting PR1 in multiple myeloma as there appears to be immunity against PR1 in these patients. The frequency of PR1-CTL in multiple patients also suggests alternative avenues for PR1-based immunotherapies. Natural PR1 immunity could potentially be boosted further using PR1 peptide vaccines. In this study, we found PR1-CTL in 
the peripheral blood and bone marrow, suggesting that these PR1-CTL have the capacity to infiltrate and home to the bone marrow tumor site. In agreement with these observations, we also noted that the majority of PR1 dextramer positive T cells aligned with the effector memory phenotype (CCR7 $/$ /CD45RA-). However, the fate of these PR1-CTL remains unknown. Further experiments are needed to assess their expansion potential and reactivity functions. Effector memory $T$ cells are characterized by their activation status, containing large quantities of perforin and IFN- $\gamma$, and being poised to readily initiate effector functions upon antigenic stimulation. ${ }^{83}$ It is tempting to speculate that the PR1-CTL we observed could function as PR1 marrow-infiltrating lymphocytes (MILs) and could further be expanded ex vivo and transferred using adoptive T cell therapy. Although, it is important to note that adoptive T cell transfer of naïve or central memory $\mathrm{T}$ cell subsets may be more ideal for a long-term anti-myeloma immunity. ${ }^{84}$

While our studies have demonstrated the feasibility of targeting PR1 in multiple myeloma, it is important to recognize potential toxicity concerns. We recognize that since PR1 is a self-antigen, there may be off target effects when utilizing PR1-targeting immunotherapies. PMN and other non-malignant myeloid cells endogenously express NE and P3 and would therefore theoretically express PR1/HLA-A2 through normal antigen presentation. However, the level of expression of PR1 by myeloid precursor cells in the bone marrow was shown to be lower than the aberrant expression by leukemia. ${ }^{50}$ This would suggest a lower density of $8 \mathrm{~F} 4$ on healthy hematopoietic cells that may be insufficient to trigger lysis of normal cells. Additionally, extensive preclinical studies and clinical trials in 
the setting of leukemia have illustrated safety and efficacy of PR1-targeting immunotherapies despite PR1 originating from the normal self antigens NE and P3. $49,50,52,68$

Additionally, the safety of targeting self-antigens such as PR1, have been supported by the ability to target other tumor-associated self antigens, such as Wilm's Tumor-1 (WT1) and NY-ESO1 ${ }^{85,86}$ Similar to PR1, WT1 has HLA-A2 restricted epitopes and is aberrantly expressed in many solid tumor and hematological malignancies, including AML. ${ }^{87}$ Importantly, WT1 has been effectively targeted in AML using WT1-specific immunotherapies, including WT1 TCR-like monoclonal antibody, WT1 CAR-T cells, and WT1 peptide vaccine, without off target toxicities. ${ }^{68,86,88-90} \mathrm{NY}$-ESO1 is another tumor-associated antigen that has been characterized as an attractive immunotherapeutic target in multiple myeloma. Despite its origins as a self-antigen, there has been interest in developing NY-ESO1 immunotherapies, with success. ${ }^{91}$ The feasibility of targeting other self-antigens indicates that targeting PR1 in multiple myeloma may also be a practical strategy.

NE and P3 are primarily localized to the azurophilic granules in mature granulocytes and $\mathrm{PMN},{ }^{92}$ sequestering them from endogenous antigen presentation by the cell. Due to this siloing, the majority of NE and P3 are not accessible for proteasomal degradation and subsequent antigen processing of the PR1 peptide. ${ }^{50}$ Moreover, transcription of NE and P3 are reduced in pro- 
myelocytes and decreases over granulocyte maturation. ${ }^{93,94}$ This spatial and temporal control over NE and P3 expression may result in low endogenous antigen presentation of PR1 by mature granulocytes, which would reduce ontarget off-tumor binding of PR1-targeting immunotherapies. Importantly, targeting PR1 has demonstrated clinical safety in the setting of leukemia. Qazilbash and colleagues conducted a phase I/II clinical trial that not only demonstrated tolerability and safety of PR1 peptide vaccine, but also showed vaccination induced specific immunity that correlated with clinical response. ${ }^{49}$

It is not surprising that we noted some level of binding of $8 \mathrm{~F} 4$ antibody to HLA-A2 molecules alone. This observation is due to the fact that HLA-A2 is part of the combined conformational PR1/HLA-A2 epitope that 8F4 antibody recognizes. We have observed low background binding in the setting of leukemia and lung cancer. ${ }^{40,41,43}$ Additionally, we did observe very low levels of background binding in unpulsed U266 multiple myeloma (Figure 26). In previous work from our group, fine epitope mapping revealed that $8 \mathrm{~F} 4$ antibody has contact residues with the helical domain of HLA-A2 molecules, which may help provide an explanation for the observed background binding by $8 \mathrm{~F} 4$ antibody. However, binding experiments using ELISA with other high affinity HLA-A2 peptides (pp65 and WT1) wherein 8F4 antibody failed to recognize these other peptide complexes, have demonstrated the specificity of $8 \mathrm{~F} 4$ antibody to PR1/HLA-A2. ${ }^{50}$ 
Overall we have described a novel mechanism by which multiple myeloma can express new tumor antigens by cross-presentation. These findings will allow for new immunotherapies to be applied to treating this malignancy. 
Figure 32: Summary of PR1 cross-presentation by multiple myeloma

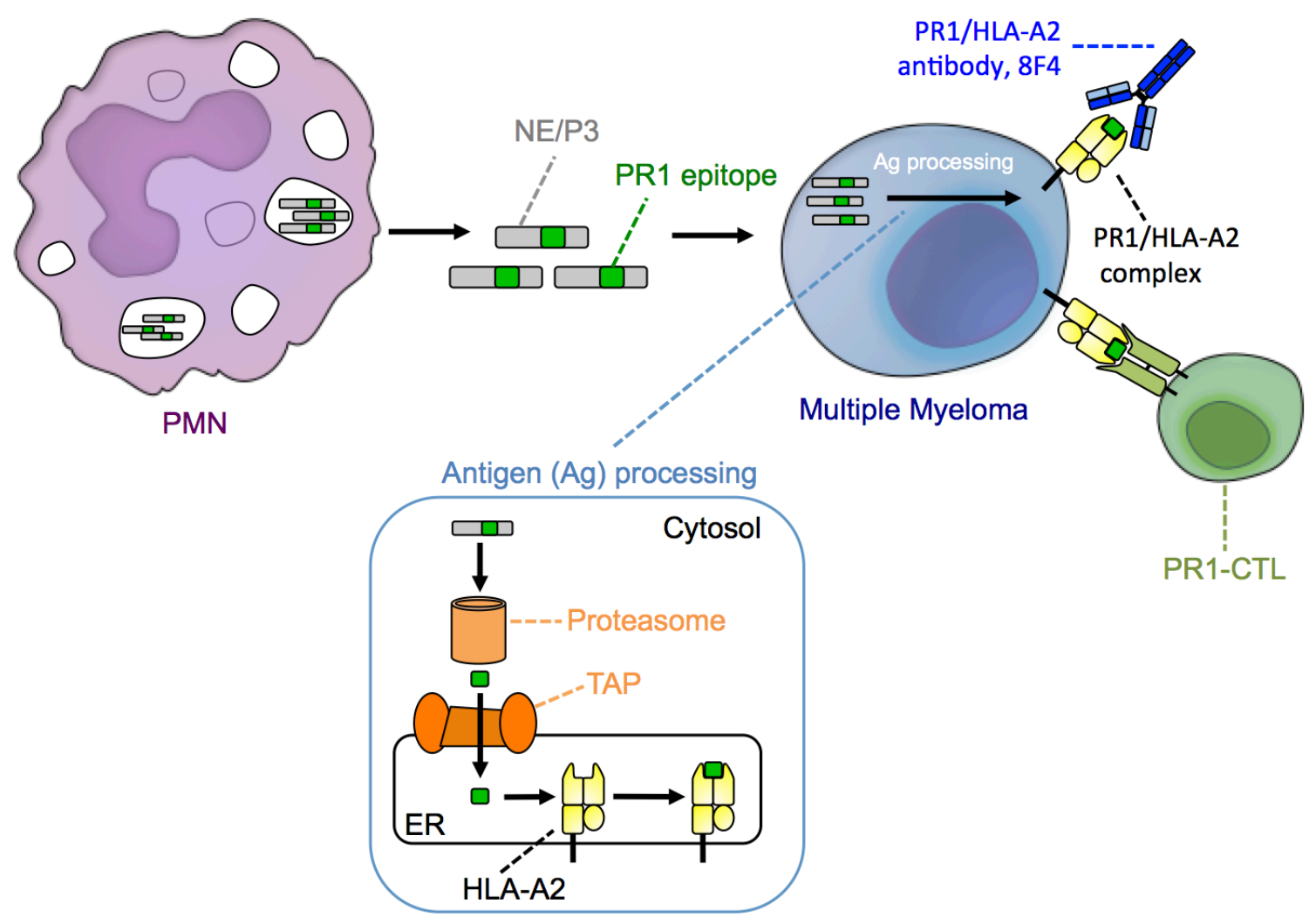

Figure 32: Summary of PR1 cross-presentation by multiple myeloma. In the bone marrow, multiple myeloma cells take up NE and P3 from resident polymorphonuclear cells (PMN). NE and P3 are then processed by the proteasome and the PR1 peptide is loaded onto HLA-A2 molecules in a TAPdependent manner in the ER. PR1/HLA-A2 complexes are then expressed on the multiple myeloma cell surface, rendering multiple myeloma susceptible to PR1-targeting immunotherapies such as $8 \mathrm{~F} 4$ antibody and PR1-CTL. 


\section{Chapter 7: Future Directions}

Our study has provided evidence that multiple myeloma is capable of taking up exogenous antigens and cross-presenting novel peptides that can be targeted by immunotherapy. However, this raises several new questions to be explored. What is the mechanism by which NE and P3 are taken up by multiple myeloma? What clinical impact does cross-presentation hold for future treatment of multiple myeloma?

The mechanism of NE and P3 uptake in hematological malignancies remains an important question. This is due in large part to the ubiquitous expression of NE and P3 in inflamed tissues, including tumors. While our group has led the investigation into the mechanism of NE uptake in solid tumors, we have yet to explore this mechanism in multiple myeloma. Kerros and colleagues have delineated a receptor-mediated mechanism for NE uptake in breast cancer. Their studies, utilizing immunoprecipitation and mass spectrometry, identified that neuropilin-1 (NRP1) is a receptor that is responsible for NE uptake in breast cancer. ${ }^{44}$ Their hypotheses were guided by two important observations. NE uptake by breast cancer was observed to be dose and time dependent and was saturable after long co-cultures. Additionally, NE was observed to localize to the early endosomal compartment at early time points, all of which suggested a receptor-mediated mechanism. ${ }^{53}$ Interestingly, our multiple myeloma uptake studies closely resemble the uptake kinetics seen breast cancer cells, suggesting 
that there may be a receptor responsible for NE uptake by multiple myeloma. However, NRP1 expression is notably absent in U266 multiple myeloma cells indicating there may be another receptor present and responsible. Similar immunoprecipitation and mass spectrometry based approaches could be employed to identify the receptor responsible for NE uptake in multiple myeloma. These findings would be important in furthering our understanding of antigenand cross-presentation by cancer cells, specifically in the setting of multiple myeloma. The mechanism of P3 uptake has yet to fully explored in solid or hematological cancers but would be another area of interesting research.

The antigen presentation machinery were shown to play a critical role in PR1 cross-presentation by multiple myeloma. We specifically investigated the essential contributions of the ER/Golgi, proteasome, and TAP. Our work also illuminated a potentially integral role of calnexin for HLA maturation in multiple myeloma patients. We observed that a lower isoform of calnexin is expressed in multiple myeloma patient cells and was correlated to reduced expression of HLA. The reduced HLA expression was also observed to resemble the immature, double-band, phenotype of HLA molecules that were retained in the ER. ${ }^{66} \mathrm{We}$ speculate that the lower calnexin isoform may function as a dominant negative chaperone, outcompeting canonical calnexin, and preventing proper HLA folding and binding to the PLC. This could be possible as there are three described isoforms of calnexin that are produced through alternative mRNA splicing, as notated in the UniProt protein database. The shortest variant of calnexin, isoform 
3 , is missing amino acids $1-108$ at the $\mathrm{N}$-terminus. This truncation in isoform 3 could potentially affect the binding of calnexin to HLA through its globular, glycan-binding domain, which is formed in part by the $\mathrm{N}$-terminus. ${ }^{95}$ Additionally, canonical length calnexin has been shown to retain HLA in the ER in TAPdeficient cells. ${ }^{96,97}$ Together with our studies, these observations suggest a possible mechanism of calnexin mediated HLA retention in the ER and improper HLA expression. However, more experimentation would be necessary to characterize this mechanism and test these hypotheses. Future investigations that explore the role of calnexin would be warranted, as from a teleological perspective, this could be novel immune escape mechanism utilized by multiple myeloma to further avoid $\mathrm{CD}^{+} \mathrm{T}$ cell detection and destruction.

The work presented here illustrates strong preclinical evidence for PR1 targeting immunotherapies for the treatment of multiple myeloma. Our investigations specifically have shown that PR1-CTL and 8F4 antibody can effectively reduce the myeloma burden and prolongs survival in xenograft animal models. Additionally, our data highlights that $8 \mathrm{~F} 4$ antibody therapy could be combined with other standard of care treatments already used for treating multiple myeloma patients such as lenalidomide and even possibly bortezomib. However, further mechanistic studies are necessary to explore how these combinatorial therapies may interact. Multiple myeloma patients commonly receive granulocyte colony stimulating factor (G-CSF) to mobilize bone marrow cells in preparation for auto-SCT. This process increases the expression of NE 
and P3 in the bone marrow and would therefore increase the availability of sources for PR1 for cross-presentation. ${ }^{48,98} 8 \mathrm{~F} 4$ antibody could be readily integrated at this stage, as a purging strategy, where multiple myeloma cells would be most likely to cross-present PR1. 8F4 would also be highly applicable to multiple myeloma patients with large disease burdens. Our pre-clinical studies in mice where high disease burdens were significantly reduced with $8 \mathrm{~F} 4$ antibody therapy alone support these speculations. In addition to 8F4 antibody, PR1 has been targeted using other modalities such as PR1 peptide vaccine and PR1-CAR T cell therapy. ${ }^{49,55}$ Each of these different strategies could be applied to multiple myeloma patients in different stages depending on the disease burden. For example, PR1 peptide vaccine could be highly valuable as a consolidation therapy post SCT or in the setting of collecting stem cells for transplant. Additionally, CAR T cell therapy may find attractive synergy when combined with checkpoint blockage.

Finally, to our knowledge, our work is the first to document that multiple myeloma can cross-present an exogenous antigen, PR1, and can be targeted for destruction using PR1-specific immunotherapies. This important finding opens up a new realm of possible exogenous tumor associated antigens to be targeted in multiple myeloma. Currently, the exploration for new cancer antigens, via surveying the HLA ligandome, has erupted in the field of cancer medicine. ${ }^{71,99-101}$ However, our studies suggest that future antigen discovery experiments should be conducted with cross-presentation in mind. Importantly, multiple myeloma has 
a low mutational burden compared to many of cancers. ${ }^{102}$ Considering that much of antigen discovery has focused on neoantigens derived from mutated antigens in both solid and hematological cancers, cross-presented peptides may be even of higher importance in the setting of multiple myeloma where neoantigens may not be as abundant as they are in other cancers. With personalized, individualbased therapy approaches becoming more of a reality, ${ }^{103,104}$ it will be even more vital to consider how tumor cells present antigens. 


\section{Bibliography}

1. Pawlyn, C. \& Morgan, G.J. Evolutionary biology of high-risk multiple myeloma. Nat Rev Cancer 17, 543-556 (2017).

2. Kuehl, W.M. \& Bergsagel, P.L. Multiple myeloma: evolving genetic events and host interactions. Nat Rev Cancer 2, 175-187 (2002).

3. Agarwal, A. \& Ghobrial, I.M. Monoclonal gammopathy of undetermined significance and smoldering multiple myeloma: a review of the current understanding of epidemiology, biology, risk stratification, and management of myeloma precursor disease. Clin Cancer Res 19, 985-994 (2013).

4. Weiss, B.M., Abadie, J., Verma, P., Howard, R.S. \& Kuehl, W.M. A monoclonal gammopathy precedes multiple myeloma in most patients. Blood 113, 5418-5422 (2009).

5. Clay-Gilmour, A.I., Kumar, S., Rajkumar, S.V., Rishi, A., Kyle, R.A., Katzmann, J.A., Murray, D.L., Norman, A.D., Greenberg, A.J., Larson, D.R., O'Byrne, M.M., Slager, S.L. \& Vachon, C.M. Risk of MGUS in relatives of multiple myeloma cases by clinical and tumor characteristics. Leukemia 33, 499-507 (2019).

6. Zhan, F., Hardin, J., Kordsmeier, B., Bumm, K., Zheng, M., Tian, E., Sanderson, R., Yang, Y., Wilson, C., Zangari, M., Anaissie, E., Morris, C., Muwalla, F., van Rhee, F., Fassas, A., Crowley, J., Tricot, G., Barlogie, B. \& Shaughnessy, J., Jr. Global gene expression profiling of multiple 
myeloma, monoclonal gammopathy of undetermined significance, and normal bone marrow plasma cells. Blood 99, 1745-1757 (2002).

7. Fonseca, R., Bailey, R.J., Ahmann, G.J., Rajkumar, S.V., Hoyer, J.D., Lust, J.A., Kyle, R.A., Gertz, M.A., Greipp, P.R. \& Dewald, G.W. Genomic abnormalities in monoclonal gammopathy of undetermined significance. Blood 100, 1417-1424 (2002).

8. Podar, K., Chauhan, D. \& Anderson, K.C. Bone marrow microenvironment and the identification of new targets for myeloma therapy. Leukemia 23, 10-24 (2009).

9. Mondello, P., Cuzzocrea, S., Navarra, M. \& Mian, M. Bone marrow microenvironment is a crucial player for myelomagenesis and disease progression. Oncotarget 8, 20394-20409 (2017).

10. Olechnowicz, S.W. \& Edwards, C.M. Contributions of the host microenvironment to cancer-induced bone disease. Cancer Res 74, 1625$1631(2014)$.

11. Fulciniti, M., Hideshima, T., Vermot-Desroches, C., Pozzi, S., Nanjappa, P., Shen, Z., Patel, N., Smith, E.S., Wang, W., Prabhala, R., Tai, Y.T., Tassone, P., Anderson, K.C. \& Munshi, N.C. A high-affinity fully human anti-IL-6 mAb, 1339, for the treatment of multiple myeloma. Clin Cancer Res 15, 7144-7152 (2009).

12. San-Miguel, J., Blade, J., Shpilberg, O., Grosicki, S., Maloisel, F., Min, C.K., Polo Zarzuela, M., Robak, T., Prasad, S.V., Tee Goh, Y., Laubach, J., Spencer, A., Mateos, M.V., Palumbo, A., Puchalski, T., Reddy, M., 
Uhlar, C., Qin, X., van de Velde, H., Xie, H. \& Orlowski, R.Z. Phase 2 randomized study of bortezomib-melphalan-prednisone with or without siltuximab (anti-IL-6) in multiple myeloma. Blood 123, 4136-4142 (2014).

13. Noonan, K. \& Borrello, I. The immune microenvironment of myeloma. Cancer Microenviron 4, 313-323 (2011).

14. Romano, A., Conticello, C., Cavalli, M., Vetro, C., La Fauci, A., Parrinello, N.L. \& Di Raimondo, F. Immunological dysregulation in multiple myeloma microenvironment. Biomed Res Int 2014, 198539 (2014).

15. Gorgun, G.T., Whitehill, G., Anderson, J.L., Hideshima, T., Maguire, C., Laubach, J., Raje, N., Munshi, N.C., Richardson, P.G. \& Anderson, K.C. Tumor-promoting immune-suppressive myeloid-derived suppressor cells in the multiple myeloma microenvironment in humans. Blood 121, 29752987 (2013).

16. Kumar, S.K., Dispenzieri, A., Lacy, M.Q., Gertz, M.A., Buadi, F.K., Pandey, S., Kapoor, P., Dingli, D., Hayman, S.R., Leung, N., Lust, J., McCurdy, A., Russell, S.J., Zeldenrust, S.R., Kyle, R.A. \& Rajkumar, S.V. Continued improvement in survival in multiple myeloma: changes in early mortality and outcomes in older patients. Leukemia 28, 1122-1128 (2014).

17. Kumar, S.K., Dispenzieri, A., Fraser, R., Mingwei, F., Akpek, G., Cornell, R., Kharfan-Dabaja, M., Freytes, C., Hashmi, S., Hildebrandt, G., Holmberg, L., Kyle, R., Lazarus, H., Lee, C., Mikhael, J., Nishihori, T., Tay, J., Usmani, S., Vesole, D., Vij, R., Wirk, B., Krishnan, A., Gasparetto, C., Mark, T., Nieto, Y., Hari, P. \& D'Souza, A. Early relapse after autologous 
hematopoietic cell transplantation remains a poor prognostic factor in multiple myeloma but outcomes have improved over time. Leukemia 32, 986-995 (2018).

18. Raab, M.S., Podar, K., Breitkreutz, I., Richardson, P.G. \& Anderson, K.C. Multiple myeloma. Lancet 374, 324-339 (2009).

19. Nunes, A.T. \& Annunziata, C.M. Proteasome inhibitors: structure and function. Semin Oncol 44, 377-380 (2017).

20. Chim, C.S., Kumar, S.K., Orlowski, R.Z., Cook, G., Richardson, P.G., Gertz, M.A., Giralt, S., Mateos, M.V., Leleu, X. \& Anderson, K.C. Management of relapsed and refractory multiple myeloma: novel agents, antibodies, immunotherapies and beyond. Leukemia 32, 252-262 (2018).

21. Gandhi, A.K., Kang, J., Havens, C.G., Conklin, T., Ning, Y., Wu, L., Ito, T., Ando, H., Waldman, M.F., Thakurta, A., Klippel, A., Handa, H., Daniel, T.O., Schafer, P.H. \& Chopra, R. Immunomodulatory agents lenalidomide and pomalidomide co-stimulate $T$ cells by inducing degradation of $T$ cell repressors Ikaros and Aiolos via modulation of the E3 ubiquitin ligase complex CRL4(CRBN.). Br J Haematol 164, 811-821 (2014).

22. Costa, F., Vescovini, R., Bolzoni, M., Marchica, V., Storti, P., Toscani, D., Accardi, F., Notarfranchi, L., Dalla Palma, B., Manferdini, C., Manni, S., Todaro, G., Lisignoli, G., Piazza, F., Aversa, F. \& Giuliani, N. Lenalidomide increases human dendritic cell maturation in multiple myeloma patients targeting monocyte differentiation and modulating 
mesenchymal stromal cell inhibitory properties. Oncotarget 8, 5305353067 (2017).

23. Moreau, P., San Miguel, J., Ludwig, H., Schouten, H., Mohty, M., Dimopoulos, M., Dreyling, M. \& Group, E.G.W. Multiple myeloma: ESMO Clinical Practice Guidelines for diagnosis, treatment and follow-up. Ann Oncol 24 Suppl 6, vi133-137 (2013).

24. Kazmi, S.M., Nusrat, M., Gunaydin, H., Cornelison, A.M., Shah, N., Kebriaei, P., Nieto, Y., Parmar, S., Popat, U.R., Oran, B., Shah, J.J., Orlowski, R.Z., Champlin, R.E., Qazilbash, M.H. \& Bashir, Q. Outcomes Among High-Risk and Standard-Risk Multiple Myeloma Patients Treated With High-Dose Chemotherapy and Autologous Hematopoietic Stem-Cell Transplantation. Clin Lymphoma Myeloma Leuk 15, 687-693 (2015).

25. Kohler, M., Greil, C., Hudecek, M., Lonial, S., Raje, N., Wasch, R. \& Engelhardt, M. Current developments in immunotherapy in the treatment of multiple myeloma. Cancer 124, 2075-2085 (2018).

26. Danylesko, I., Beider, K., Shimoni, A. \& Nagler, A. Novel strategies for immunotherapy in multiple myeloma: previous experience and future directions. Clin Dev Immunol 2012, 753407 (2012).

27. Krejcik, J., Casneuf, T., Nijhof, I.S., Verbist, B., Bald, J., Plesner, T., Syed, K., Liu, K., van de Donk, N.W., Weiss, B.M., Ahmadi, T., Lokhorst, H.M., Mutis, T. \& Sasser, A.K. Daratumumab depletes CD38+ immune regulatory cells, promotes T-cell expansion, and skews T-cell repertoire in multiple myeloma. Blood 128, 384-394 (2016). 
28. van de Donk, N., Richardson, P.G. \& Malavasi, F. CD38 antibodies in multiple myeloma: back to the future. Blood 131, 13-29 (2018).

29. Lonial, S., Dimopoulos, M., Palumbo, A., White, D., Grosicki, S., Spicka, I., Walter-Croneck, A., Moreau, P., Mateos, M.V., Magen, H., Belch, A., Reece, D., Beksac, M., Spencer, A., Oakervee, H., Orlowski, R.Z., Taniwaki, M., Rollig, C., Einsele, H., Wu, K.L., Singhal, A., San-Miguel, J., Matsumoto, M., Katz, J., Bleickardt, E., Poulart, V., Anderson, K.C., Richardson, P. \& Investigators, E.-. Elotuzumab Therapy for Relapsed or Refractory Multiple Myeloma. N Engl J Med 373, 621-631 (2015).

30. Franssen, L.E., Mutis, T., Lokhorst, H.M. \& van de Donk, N. Immunotherapy in myeloma: how far have we come? Ther Adv Hematol 10, 2040620718822660 (2019).

31. Atanackovic, D., Luetkens, T. \& Kroger, N. Coinhibitory molecule PD-1 as a potential target for the immunotherapy of multiple myeloma. Leukemia 28, 993-1000 (2014).

32. Rabinovich, G.A., Gabrilovich, D. \& Sotomayor, E.M. Immunosuppressive strategies that are mediated by tumor cells. Annu Rev Immunol 25, 267296 (2007).

33. Racanelli, V., Leone, P., Frassanito, M.A., Brunetti, C., Perosa, F., Ferrone, S. \& Dammacco, F. Alterations in the antigen processingpresenting machinery of transformed plasma cells are associated with reduced recognition by $\mathrm{CD} 8+\mathrm{T}$ cells and characterize the progression of MGUS to multiple myeloma. Blood 115, 1185-1193 (2010). 
34. Alatrash, G., Molldrem, J.J. \& Qazilbash, M.H. Targeting PR1 in myeloid leukemia. Oncotarget 9, 4280-4281 (2018).

35. Grotzke, J.E., Sengupta, D., Lu, Q. \& Cresswell, P. The ongoing saga of the mechanism(s) of MHC class I-restricted cross-presentation. Curr Opin Immunol 46, 89-96 (2017).

36. Joffre, O.P., Segura, E., Savina, A. \& Amigorena, S. Cross-presentation by dendritic cells. Nat Rev Immunol 12, 557-569 (2012).

37. Ackerman, A.L. \& Cresswell, P. Cellular mechanisms governing crosspresentation of exogenous antigens. Nat Immunol 5, 678-684 (2004).

38. Cresswell, P., Ackerman, A.L., Giodini, A., Peaper, D.R. \& Wearsch, P.A. Mechanisms of MHC class I-restricted antigen processing and crosspresentation. Immunol Rev 207, 145-157 (2005).

39. Lizee, G., Basha, G., Tiong, J., Julien, J.P., Tian, M., Biron, K.E. \& Jefferies, W.A. Control of dendritic cell cross-presentation by the major histocompatibility complex class I cytoplasmic domain. Nat Immunol 4, 1065-1073 (2003).

40. Alatrash, G., Mittendorf, E.A., Sergeeva, A., Sukhumalchandra, P., Qiao, N., Zhang, M., St John, L.S., Ruisaard, K., Haugen, C.E., Al-Atrache, Z., Jakher, H., Philips, A.V., Ding, X., Chen, J.Q., Wu, Y., Patenia, R.S., Bernatchez, C., Vence, L.M., Radvanyi, L.G., Hwu, P., Clise-Dwyer, K., Ma, Q., Lu, S. \& Molldrem, J.J. Broad Cross-Presentation of the Hematopoietically Derived PR1 Antigen on Solid Tumors Leads to 
Susceptibility to PR1-Targeted Immunotherapy. J. Immunol. 189, 5476$5484(2012)$.

41. Alatrash, G., Ono, Y., Sergeeva, A., Sukhumalchandra, P., Zhang, M., St John, L.S., Yang, T.H., Ruisaard, K., Armistead, P.M., Mittendorf, E.A., He, H., Qiao, N., Rodriguez-Cruz, T., Liang, S., Clise-Dwyer, K., Wieder, E.D., Lizee, G., Lu, S. \& Molldrem, J.J. The role of antigen crosspresentation from leukemia blasts on immunity to the leukemia-associated antigen PR1. J. Immunother. 35, 309-320 (2012).

42. Alatrash, G., Perakis, A.A., Kerros, C., Peters, H.L., Sukhumalchandra, P., Zhang, M., Jakher, H., Zope, M., Patenia, R., Sergeeva, A., Yi, S., Young, K.H., Philips, A.V., Cernosek, A.M., Garber, H.R., Qiao, N., Weng, J., St John, L.S., Lu, S., Clise-Dwyer, K., Mittendorf, E.A., Ma, Q. \& Molldrem, J.J. Targeting the Leukemia Antigen PR1 with Immunotherapy for the Treatment of Multiple Myeloma. Clin Cancer Res 24, 3386-3396 (2018).

43. Peters, H.L., Tripathi, S.C., Kerros, C., Katayama, H., Garber, H.R., St John, L.S., Federico, L., Meraz, I.M., Roth, J.A., Sepesi, B., Majidi, M., Ruisaard, K., Clise-Dwyer, K., Roszik, J., Gibbons, D.L., Heymach, J.V., Swisher, S.G., Bernatchez, C., Alatrash, G., Hanash, S. \& Molldrem, J.J. Serine Proteases Enhance Immunogenic Antigen Presentation on Lung Cancer Cells. Cancer Immunol Res 5, 319-329 (2017).

44. Kerros, C., Tripathi, S.C., Zha, D., Mehrens, J.M., Sergeeva, A., Philips, A.V., Qiao, N., Peters, H.L., Katayama, H., Sukhumalchandra, P., Ruisaard, K.E., Perakis, A.A., St John, L.S., Lu, S., Mittendorf, E.A., Clise- 
Dwyer, K., Herrmann, A.C., Alatrash, G., Toniatti, C., Hanash, S.M., Ma, Q. \& Molldrem, J.J. Neuropilin-1 mediates neutrophil elastase uptake and cross-presentation in breast cancer cells. J Biol Chem 292, 10295-10305 (2017).

45. Houghton, A.M., Rzymkiewicz, D.M., Ji, H., Gregory, A.D., Egea, E.E., Metz, H.E., Stolz, D.B., Land, S.R., Marconcini, L.A., Kliment, C.R., Jenkins, K.M., Beaulieu, K.A., Mouded, M., Frank, S.J., Wong, K.K. \& Shapiro, S.D. Neutrophil elastase-mediated degradation of IRS-1 accelerates lung tumor growth. Nat Med 16, 219-223 (2010).

46. Borregaard, N. Development of neutrophil granule diversity. Ann N Y Acad Sci 832, 62-68 (1997).

47. El-Ouriaghli, F., Sloand, E., Mainwaring, L., Fujiwara, H., Keyvanfar, K., Melenhorst, J.J., Rezvani, K., Sconocchia, G., Solomon, S., Hensel, N. \& Barrett, A.J. Clonal dominance of chronic myelogenous leukemia is associated with diminished sensitivity to the antiproliferative effects of neutrophil elastase. Blood 102, 3786-3792 (2003).

48. Garwicz, D., Lennartsson, A., Jacobsen, S.E., Gullberg, U. \& Lindmark, A. Biosynthetic profiles of neutrophil serine proteases in a human bone marrow-derived cellular myeloid differentiation model. Haematologica 90, 38-44 (2005).

49. Qazilbash, M.H., Wieder, E., Thall, P.F., Wang, X., Rios, R., Lu, S., Kanodia, S., Ruisaard, K.E., Giralt, S.A., Estey, E.H., Cortes, J., Komanduri, K.V., Clise-Dwyer, K., Alatrash, G., Ma, Q., Champlin, R.E. \& 
Molldrem, J.J. PR1 peptide vaccine induces specific immunity with clinical responses in myeloid malignancies. Leukemia 31, 697-704 (2017).

50. Sergeeva, A., Alatrash, G., He, H., Ruisaard, K., Lu, S., Wygant, J., McIntyre, B.W., Ma, Q., Li, D., St John, L., Clise-Dwyer, K. \& Molldrem, J.J. An anti-PR1/HLA-A2 T-cell receptor-like antibody mediates complement-dependent cytotoxicity against acute myeloid leukemia progenitor cells. Blood 117, 4262-4272 (2011).

51. Stone, J.D., Chervin, A.S. \& Kranz, D.M. T-cell receptor binding affinities and kinetics: impact on T-cell activity and specificity. Immunology 126, 165-176 (2009).

52. Sergeeva, A., He, H., Ruisaard, K., St John, L., Alatrash, G., Clise-Dwyer, K., Li, D., Patenia, R., Hong, R., Sukhumalchandra, P., You, M.J., Gagea, M., Ma, Q. \& Molldrem, J.J. Activity of 8F4, a T-cell receptor-like antiPR1/HLA-A2 antibody, against primary human AML in vivo. Leukemia 30, 1475-1484 (2016).

53. Mittendorf, E.A., Alatrash, G., Qiao, N., Wu, Y., Sukhumalchandra, P., St John, L.S., Philips, A.V., Xiao, H., Zhang, M., Ruisaard, K., Clise-Dwyer, K., Lu, S. \& Molldrem, J.J. Breast cancer cell uptake of the inflammatory mediator neutrophil elastase triggers an anticancer adaptive immune response. Cancer Res 72, 3153-3162 (2012).

54. Miyakawa, Y., Ohnishi, Y., Tomisawa, M., Monnai, M., Kohmura, K., Ueyama, Y., Ito, M., Ikeda, Y., Kizaki, M. \& Nakamura, M. Establishment of a new model of human multiple myeloma using 
NOD/SCID/gammac(null) (NOG) mice. Biochem Biophys Res Commun 313, 258-262 (2004).

55. Ma, Q., Garber, H.R., Lu, S., He, H., Tallis, E., Ding, X., Sergeeva, A., Wood, M.S., Dotti, G., Salvado, B., Ruisaard, K., Clise-Dwyer, K., John, L.S., Rezvani, K., Alatrash, G., Shpall, E.J. \& Molldrem, J.J. A novel TCRlike CAR with specificity for PR1/HLA-A2 effectively targets myeloid leukemia in vitro when expressed in human adult peripheral blood and cord blood T cells. Cytotherapy 18, 985-994 (2016).

56. Molldrem, J., Dermime, S., Parker, K., Jiang, Y.Z., Mavroudis, D., Hensel, N., Fukushima, P. \& Barrett, A.J. Targeted T-cell therapy for human leukemia: cytotoxic T lymphocytes specific for a peptide derived from proteinase 3 preferentially lyse human myeloid leukemia cells. Blood $\mathbf{8 8}$, 2450-2457 (1996).

57. Molldrem, J.J., Lee, P.P., Wang, C., Felio, K., Kantarjian, H.M., Champlin, R.E. \& Davis, M.M. Evidence that specific T lymphocytes may participate in the elimination of chronic myelogenous leukemia. Nat Med 6, 10181023 (2000).

58. St John, L.S., Wan, L., He, H., Garber, H.R., Clise-Dwyer, K., Alatrash, G., Rezvani, K., Shpall, E.J., Bollard, C.M., Ma, Q. \& Molldrem, J.J. PR1specific cytotoxic $\mathrm{T}$ lymphocytes are relatively frequent in umbilical cord blood and can be effectively expanded to target myeloid leukemia. Cytotherapy 18, 995-1001 (2016). 
59. Barretina, J., Caponigro, G., Stransky, N., Venkatesan, K., Margolin, A.A., Kim, S., Wilson, C.J., Lehar, J., Kryukov, G.V., Sonkin, D., Reddy, A., Liu, M., Murray, L., Berger, M.F., Monahan, J.E., Morais, P., Meltzer, J., Korejwa, A., Jane-Valbuena, J., Mapa, F.A., Thibault, J., Bric-Furlong, E., Raman, P., Shipway, A., Engels, I.H., Cheng, J., Yu, G.K., Yu, J., Aspesi, P., Jr., de Silva, M., Jagtap, K., Jones, M.D., Wang, L., Hatton, C., Palescandolo, E., Gupta, S., Mahan, S., Sougnez, C., Onofrio, R.C., Liefeld, T., MacConaill, L., Winckler, W., Reich, M., Li, N., Mesirov, J.P., Gabriel, S.B., Getz, G., Ardlie, K., Chan, V., Myer, V.E., Weber, B.L., Porter, J., Warmuth, M., Finan, P., Harris, J.L., Meyerson, M., Golub, T.R., Morrissey, M.P., Sellers, W.R., Schlegel, R. \& Garraway, L.A. The Cancer Cell Line Encyclopedia enables predictive modelling of anticancer drug sensitivity. Nature 483, 603-607 (2012).

60. Borregaard, N. \& Cowland, J.B. Granules of the human neutrophilic polymorphonuclear leukocyte. Blood 89, 3503-3521 (1997).

61. Gregory, A.D., Hale, P., Perlmutter, D.H. \& Houghton, A.M. Clathrin pitmediated endocytosis of neutrophil elastase and cathepsin $\mathrm{G}$ by cancer cells. The Journal of biological chemistry 287, 35341-35350 (2012).

62. Houghton, A.M., Rzymkiewicz, D.M., Ji, H., Gregory, A.D., Egea, E.E., Metz, H.E., Stolz, D.B., Land, S.R., Marconcini, L.A., Kliment, C.R., Jenkins, K.M., Beaulieu, K.A., Mouded, M., Frank, S.J., Wong, K.K. \& Shapiro, S.D. Neutrophil elastase-mediated degradation of IRS-1 accelerates lung tumor growth. Nat. Med. 16, 219-223 (2010). 
63. Kovacsovics-Bankowski, M. \& Rock, K.L. A phagosome-to-cytosol pathway for exogenous antigens presented on MHC class I molecules. Science 267, 243-246 (1995).

64. Francois, M., Romieu-Mourez, R., Stock-Martineau, S., Boivin, M.N., Bramson, J.L. \& Galipeau, J. Mesenchymal stromal cells cross-present soluble exogenous antigens as part of their antigen-presenting cell properties. Blood 114, 2632-2638 (2009).

65. Mukai, T., Maeda, Y., Tamura, T., Matsuoka, M., Tsukamoto, Y. \& Makino, M. Induction of cross-priming of naive CD8+ T lymphocytes by recombinant bacillus Calmette-Guerin that secretes heat shock protein 70major membrane protein-II fusion protein. J. Immunol. 183, 6561-6568 (2009).

66. Paulsson, K.M., Jevon, M., Wang, J.W., Li, S. \& Wang, P. The double lysine motif of tapasin is a retrieval signal for retention of unstable MHC class I molecules in the endoplasmic reticulum. J Immunol 176, 74827488 (2006).

67. Rezvani, K., Grube, M., Brenchley, J.M., Sconocchia, G., Fujiwara, H., Price, D.A., Gostick, E., Yamada, K., Melenhorst, J., Childs, R., Hensel, N., Douek, D.C. \& Barrett, A.J. Functional leukemia-associated antigenspecific memory CD8+ T cells exist in healthy individuals and in patients with chronic myelogenous leukemia before and after stem cell transplantation. Blood 102, 2892-2900 (2003). 
68. Rezvani, K., Yong, A.S., Mielke, S., Savani, B.N., Musse, L., Superata, J., Jafarpour, B., Boss, C. \& Barrett, A.J. Leukemia-associated antigenspecific T-cell responses following combined PR1 and WT1 peptide vaccination in patients with myeloid malignancies. Blood 111, 236-242 (2008).

69. Lacey, S.F., La Rosa, C., Kaltcheva, T., Srivastava, T., Seidel, A., Zhou, W., Rawal, R., Hagen, K., Krishnan, A., Longmate, J., Andersson, H.A., St John, L., Bhatia, R., Pullarkat, V., Forman, S.J., Cooper, L.J., Molldrem, J. \& Diamond, D.J. Characterization of immunologic properties of a second HLA-A2 epitope from a granule protease in CML patients and HLA-A2 transgenic mice. Blood 118, 2159-2169 (2011).

70. Molldrem, J.J., Lee, P.P., Wang, C., Champlin, R.E. \& Davis, M.M. A PR1human leukocyte antigen-A2 tetramer can be used to isolate lowfrequency cytotoxic $\mathrm{T}$ lymphocytes from healthy donors that selectively lyse chronic myelogenous leukemia. Cancer Res. 59, 2675-2681 (1999).

71. Walz, S., Stickel, J.S., Kowalewski, D.J., Schuster, H., Weisel, K., Backert, L., Kahn, S., Nelde, A., Stroh, T., Handel, M., Kohlbacher, O., Kanz, L., Salih, H.R., Rammensee, H.G. \& Stevanovic, S. The antigenic landscape of multiple myeloma: mass spectrometry (re)defines targets for T-cellbased immunotherapy. Blood 126, 1203-1213 (2015).

72. Jung, S., Unutmaz, D., Wong, P., Sano, G., De los Santos, K., Sparwasser, T., Wu, S., Vuthoori, S., Ko, K., Zavala, F., Pamer, E.G., Littman, D.R. \& Lang, R.A. In vivo depletion of CD11c+ dendritic cells 
abrogates priming of $\mathrm{CD} 8+\mathrm{T}$ cells by exogenous cell-associated antigens. Immunity 17, 211-220 (2002).

73. McDonnell, A.M., Joost Lesterhuis, W., Khong, A., Nowak, A.K., Lake, R.A., Currie, A.J. \& Robinson, B.W. Restoration of defective crosspresentation in tumors by gemcitabine. Oncoimmunology 4, e1005501 (2015).

74. Marino, E., Tan, B., Binge, L., Mackay, C.R. \& Grey, S.T. B-cell crosspresentation of autologous antigen precipitates diabetes. Diabetes 61, 2893-2905 (2012).

75. Jiang, W., Lederman, M.M., Harding, C.V. \& Sieg, S.F. Presentation of soluble antigens to CD8+ T cells by CpG oligodeoxynucleotide-primed human naive B cells. J Immunol 186, 2080-2086 (2011).

76. Yi, Q., Dabadghao, S., Osterborg, A., Bergenbrant, S. \& Holm, G. Myeloma bone marrow plasma cells: evidence for their capacity as antigen-presenting cells. Blood 90, 1960-1967 (1997).

77. Perez-Andres, M., Almeida, J., Martin-Ayuso, M., Moro, M.J., MartinNunez, G., Galende, J., Borrego, D., Rodriguez, M.J., Ortega, F., Hernandez, J., Moreno, I., Dominguez, M., Mateo, G., San Miguel, J.F., Orfao, A., Spanish Network on multiple, m. \& Spanish Network of Cancer Research, C. Clonal plasma cells from monoclonal gammopathy of undetermined significance, multiple myeloma and plasma cell leukemia show different expression profiles of molecules involved in the interaction 
with the immunological bone marrow microenvironment. Leukemia 19, 449-455 (2005).

78. Milner, E., Gutter-Kapon, L., Bassani-Strenberg, M., Barnea, E., Beer, I. \& Admon, A. The effect of proteasome inhibition on the generation of the human leukocyte antigen (HLA) peptidome. Mol Cell Proteomics 12, 1853$1864(2013)$.

79. Destro, F., Sforza, F., Sicurella, M., Marescotti, D., Gallerani, E., Baldisserotto, A., Marastoni, M. \& Gavioli, R. Proteasome inhibitors induce the presentation of an Epstein-Barr virus nuclear antigen 1-derived cytotoxic T lymphocyte epitope in Burkitt's lymphoma cells. Immunology 133, 105-114 (2011).

80. Rock, K.L., York, I.A. \& Goldberg, A.L. Post-proteasomal antigen processing for major histocompatibility complex class I presentation. Nat Immunol 5, 670-677 (2004).

81. Ma, W., Zhang, Y., Vigneron, N., Stroobant, V., Thielemans, K., van der Bruggen, P. \& Van den Eynde, B.J. Long-Peptide Cross-Presentation by Human Dendritic Cells Occurs in Vacuoles by Peptide Exchange on Nascent MHC Class I Molecules. J Immunol 196, 1711-1720 (2016).

82. Ma, W., Stroobant, V., Heirman, C., Sun, Z., Thielemans, K., Mulder, A., van der Bruggen, P. \& Van den Eynde, B.J. The Vacuolar Pathway of Long Peptide Cross-Presentation Can Be TAP Dependent. J Immunol 202, 451-459 (2019). 
83. Sallusto, F., Geginat, J. \& Lanzavecchia, A. Central memory and effector memory T cell subsets: function, generation, and maintenance. Annu Rev Immunol 22, 745-763 (2004).

84. Klebanoff, C.A., Scott, C.D., Leonardi, A.J., Yamamoto, T.N., Cruz, A.C., Ouyang, C., Ramaswamy, M., Roychoudhuri, R., Ji, Y., Eil, R.L., Sukumar, M., Crompton, J.G., Palmer, D.C., Borman, Z.A., Clever, D., Thomas, S.K., Patel, S., Yu, Z., Muranski, P., Liu, H., Wang, E., Marincola, F.M., Gros, A., Gattinoni, L., Rosenberg, S.A., Siegel, R.M. \& Restifo, N.P. Memory T cell-driven differentiation of naive cells impairs adoptive immunotherapy. J Clin Invest 126, 318-334 (2016).

85. Held, G., Matsuo, M., Epel, M., Gnjatic, S., Ritter, G., Lee, S.Y., Tai, T.Y., Cohen, C.J., Old, L.J., Pfreundschuh, M., Reiter, Y., Hoogenboom, H.R. \& Renner, C. Dissecting cytotoxic T cell responses towards the NY-ESO-1 protein by peptide/MHC-specific antibody fragments. Eur J Immunol 34, 2919-2929 (2004).

86. Rafiq, S., Dao, T., Liu, C., Scheinberg, D.A. \& Brentjens, R.J. Engineered T Cell Receptor-Mimic Antibody, (TCRm) Chimeric Antigen Receptor (CAR) T Cells Against the Intracellular Protein Wilms Tumor-1 (WT1) for Treatment of Hematologic and Solid Cancers. Blood 124, 2155-2155 (2014).

87. Jaigirdar, A., Rosenberg, S.A. \& Parkhurst, M. A High-avidity WT1reactive T-Cell Receptor Mediates Recognition of Peptide and Processed 
Antigen but not Naturally Occurring WT1-positive Tumor Cells. $J$ Immunother 39, 105-116 (2016).

88. Di Stasi, A., Jimenez, A.M., Minagawa, K., Al-Obaidi, M. \& Rezvani, K. Review of the Results of WT1 Peptide Vaccination Strategies for Myelodysplastic Syndromes and Acute Myeloid Leukemia from Nine Different Studies. Front Immuno/ 6, 36 (2015).

89. Rezvani, K., Yong, A.S., Mielke, S., Jafarpour, B., Savani, B.N., Le, R.Q., Eniafe, R., Musse, L., Boss, C., Kurlander, R. \& Barrett, A.J. Repeated PR1 and WT1 peptide vaccination in Montanide-adjuvant fails to induce sustained high-avidity, epitope-specific CD8+ T cells in myeloid malignancies. Haematologica 96, 432-440 (2011).

90. Rafiq, S., Purdon, T.J., Daniyan, A.F., Koneru, M., Dao, T., Liu, C., Scheinberg, D.A. \& Brentjens, R.J. Optimized T-cell receptor-mimic chimeric antigen receptor T cells directed toward the intracellular Wilms Tumor 1 antigen. Leukemia 31, 1788-1797 (2017).

91. Rapoport, A.P., Stadtmauer, E.A., Binder-Scholl, G.K., Goloubeva, O., Vogl, D.T., Lacey, S.F., Badros, A.Z., Garfall, A., Weiss, B., Finklestein, J., Kulikovskaya, I., Sinha, S.K., Kronsberg, S., Gupta, M., Bond, S., Melchiori, L., Brewer, J.E., Bennett, A.D., Gerry, A.B., Pumphrey, N.J., Williams, D., Tayton-Martin, H.K., Ribeiro, L., Holdich, T., Yanovich, S., Hardy, N., Yared, J., Kerr, N., Philip, S., Westphal, S., Siegel, D.L., Levine, B.L., Jakobsen, B.K., Kalos, M. \& June, C.H. NY-ESO-1-specific 
TCR-engineered T cells mediate sustained antigen-specific antitumor effects in myeloma. Nat Med 21, 914-921 (2015).

92. Egesten, A., Breton-Gorius, J., Guichard, J., Gullberg, U. \& Olsson, I. The heterogeneity of azurophil granules in neutrophil promyelocytes: immunogold localization of myeloperoxidase, cathepsin G, elastase, proteinase 3 , and bactericidal/permeability increasing protein. Blood $\mathbf{8 3}$, 2985-2994 (1994).

93. Sturrock, A., Franklin, K.F. \& Hoidal, J.R. Human proteinase-3 expression is regulated by PU.1 in conjunction with a cytidine-rich element. $J$ Biol Chem 271, 32392-32402 (1996).

94. Zimmer, M., Medcalf, R.L., Fink, T.M., Mattmann, C., Lichter, P. \& Jenne, D.E. Three human elastase-like genes coordinately expressed in the myelomonocyte lineage are organized as a single genetic locus on 19pter. Proc Natl Acad Sci U S A 89, 8215-8219 (1992).

95. Schrag, J.D., Bergeron, J.J., Li, Y., Borisova, S., Hahn, M., Thomas, D.Y. \& Cygler, M. The Structure of calnexin, an ER chaperone involved in quality control of protein folding. Mol Cell 8, 633-644 (2001).

96. Rajagopalan, S. \& Brenner, M.B. Calnexin retains unassembled major histocompatibility complex class I free heavy chains in the endoplasmic reticulum. J Exp Med 180, 407-412 (1994).

97. Jackson, M.R., Cohen-Doyle, M.F., Peterson, P.A. \& Williams, D.B. Regulation of MHC class I transport by the molecular chaperone, calnexin (p88, IP90). Science 263, 384-387 (1994). 
98. Lieschke, G.J., Grail, D., Hodgson, G., Metcalf, D., Stanley, E., Cheers, C., Fowler, K.J., Basu, S., Zhan, Y.F. \& Dunn, A.R. Mice lacking granulocyte colony-stimulating factor have chronic neutropenia, granulocyte and macrophage progenitor cell deficiency, and impaired neutrophil mobilization. Blood 84, 1737-1746 (1994).

99. Kowalewski, D.J., Schuster, H., Backert, L., Berlin, C., Kahn, S., Kanz, L., Salih, H.R., Rammensee, H.G., Stevanovic, S. \& Stickel, J.S. HLA ligandome analysis identifies the underlying specificities of spontaneous antileukemia immune responses in chronic lymphocytic leukemia (CLL). Proc Natl Acad Sci U S A 112, E166-175 (2015).

100. Loffler, M.W., Kowalewski, D.J., Backert, L., Bernhardt, J., Adam, P., Schuster, H., Dengler, F., Backes, D., Kopp, H.G., Beckert, S., Wagner, S., Konigsrainer, I., Kohlbacher, O., Kanz, L., Konigsrainer, A., Rammensee, H.G., Stevanovic, S. \& Haen, S.P. Mapping the HLA Ligandome of Colorectal Cancer Reveals an Imprint of Malignant Cell Transformation. Cancer Res 78, 4627-4641 (2018).

101. Berlin, C., Kowalewski, D.J., Schuster, H., Mirza, N., Walz, S., Handel, M., Schmid-Horch, B., Salih, H.R., Kanz, L., Rammensee, H.G., Stevanovic, S. \& Stickel, J.S. Mapping the HLA ligandome landscape of acute myeloid leukemia: a targeted approach toward peptide-based immunotherapy. Leukemia 29, 647-659 (2015).

102. Alexandrov, L.B., Nik-Zainal, S., Wedge, D.C., Aparicio, S.A., Behjati, S., Biankin, A.V., Bignell, G.R., Bolli, N., Borg, A., Borresen-Dale, A.L., 
Boyault, S., Burkhardt, B., Butler, A.P., Caldas, C., Davies, H.R., Desmedt, C., Eils, R., Eyfjord, J.E., Foekens, J.A., Greaves, M., Hosoda, F., Hutter, B., Ilicic, T., Imbeaud, S., Imielinski, M., Jager, N., Jones, D.T., Jones, D., Knappskog, S., Kool, M., Lakhani, S.R., Lopez-Otin, C., Martin, S., Munshi, N.C., Nakamura, H., Northcott, P.A., Pajic, M., Papaemmanuil, E., Paradiso, A., Pearson, J.V., Puente, X.S., Raine, K., Ramakrishna, M., Richardson, A.L., Richter, J., Rosenstiel, P., Schlesner, M., Schumacher, T.N., Span, P.N., Teague, J.W., Totoki, Y., Tutt, A.N., Valdes-Mas, R., van Buuren, M.M., van 't Veer, L., Vincent-Salomon, A., Waddell, N., Yates, L.R., Australian Pancreatic Cancer Genome, I., Consortium, I.B.C., Consortium, I.M.-S., PedBrain, I., Zucman-Rossi, J., Futreal, P.A., McDermott, U., Lichter, P., Meyerson, M., Grimmond, S.M., Siebert, R., Campo, E., Shibata, T., Pfister, S.M., Campbell, P.J. \& Stratton, M.R. Signatures of mutational processes in human cancer. Nature 500, 415$421(2013)$.

103. van Rooij, N., van Buuren, M.M., Philips, D., Velds, A., Toebes, M., Heemskerk, B., van Dijk, L.J., Behjati, S., Hilkmann, H., El Atmioui, D., Nieuwland, M., Stratton, M.R., Kerkhoven, R.M., Kesmir, C., Haanen, J.B., Kvistborg, P. \& Schumacher, T.N. Tumor exome analysis reveals neoantigen-specific T-cell reactivity in an ipilimumab-responsive melanoma. J Clin Oncol 31, e439-442 (2013).

104. Robbins, P.F., Lu, Y.C., El-Gamil, M., Li, Y.F., Gross, C., Gartner, J., Lin, J.C., Teer, J.K., Cliften, P., Tycksen, E., Samuels, Y. \& Rosenberg, S.A. 
Mining exomic sequencing data to identify mutated antigens recognized by adoptively transferred tumor-reactive T cells. Nat Med 19, 747-752 (2013). 


\section{Vita}

Alexander A. Perakis was born in Houston, Texas, the son of Alexander George Perakis and Patty Perakis. He moved to Colorado with his family in 2006 and graduated from Thunder Ridge High School in Highlands Ranch, Colorado in 2009. He received his Bachelor of Arts with a degree in Molecular, Cellular, and Developmental Biology from the University of Colorado, Boulder in 2013. He moved back to Houston, Texas in August 2013 and matriculated to the University of Texas MD Anderson Cancer Center UTHealth Graduate School of Biomedical Sciences. 